\title{
Mean field propagation of infinite-dimensional Wigner measures with a singular two-body interaction potential
}

\author{
ZIED AMMARI AND FRANCIS NIER
}

\begin{abstract}
We consider the quantum dynamics of many bosons systems in the mean field limit with a singular pair-interaction potential, including the attractive or repulsive Coulombic case in three dimensions. By using a measure transportation technique developed in [3], we show that Wigner measures propagate along the nonlinear Hartree flow. Such property was previously proved only for bounded potentials in our works $[5,6]$ with a slightly different strategy.
\end{abstract}

Mathematics Subject Classification (2010): $81 \mathrm{~S} 30$ (primary); 81S05, 81T10, 35Q55, 28A33 (secondary).

\section{Introduction}

The evolution of a non-relativistic system of many quantum particles is described by an $n$-body Schrödinger equation. The mean field limit consists in replacing this problem by a non linear 1-particle problem, by considering one generic particle interacting with the average field of all the others, when the number of particles is large and the interaction potential is weak. It is common knowledge that this approximation starts to be very effective when the number of particles exceeds a few tens. In the last decades, many works have been devoted to justify this limit. Most of them considered the mean field dynamics of well-prepared quantum states, coherent states or Hermite states, by following and extending the phase-space approach, also known as the Hepp method (see $[25,27,32,33,39,44,58]$ ), or by studying the BBGKY hierarchy of reduced density matrices (see $[8,17,19,20,42,60])$. Some of these results deal with very singular pair interaction potentials in $[9,19,20,44]$ or consider the rate of convergence (see $[7,44,58]$ ), sometimes motivated by the modelling of Bose-Einstein condensates (see e.g. $[1,21,48]$ ). In this article we continue our program, which consists in deriving the mean field limit for general initial data in the bosonic framework. Our strategy is inspired by older attempts to give substance to the formal link between bosonic Quantum Field Theory and the finitedimensional microlocal or phase-space analysis (see $[10,23,24,45,47])$. With this 
respect, the small parameter $\varepsilon=\frac{1}{n}$ asymptotics is the infinite-dimensional version of semiclassical analysis. And it has been realized in the 90's that the Wigner (or semiclassical) measures provide a powerful tool in order to obtain the leading term in the semiclassical limit (see $[29,30,37,49])$, because they flexibly and efficiently incorporate a priori estimates (see [13,14,22,51,54]).

In [4] Wigner measures were introduced in the infinite-dimensional setting and their main properties were studied. The above-mentioned work exploited and clarified the intimate relationship between pseudo-differential calculus, phase-space geometry and the probability approach, inherent to bosonic QFT. In [5], the dynamics for well-prepared data and bounded interaction potentials was reconsidered within this approach. The general propagation result was obtained in [6] for bounded interaction potentials. In particular, we showed that the BBGKY hierarchy dynamics is a projected picture of the evolution of the Wigner measure, for which there is a closed equation. One difficulty that was solved in [6] is concerned with the integration of a weak Liouville equation valid after testing with cylindrical or polynomial observables: Such classes of observables are not preserved by the nonlinear Hamiltonian mean field flow. For bounded interaction potentials, the number conservation allows polynomial approximations of the nonlinear deformation in balls of the phasespace. This is done by adapting a truncated Dyson expansion approach presented in [25-27]. In applications, an important case is the 2-body Coulomb interaction since it models the general non-relativistic motion of charged (or gravitational) particles. Again there are results about the mean field problem for specific initial data (see $[9,44])$, but the approach we have followed in [6] essentially fails. With a singular pair interaction potential, a solution to this problem is provided by measure transportation techniques developed for optimal transport theory (see $[3,62]$ ). Hence, the dynamical mean field limit relies even more on the fact that Wigner measures are probability measures on the phase-space.

We now expose our main result. The Hamiltonian of an $n$-body quantum system, with a pair interaction potential, is given by the Schrödinger operator

$$
H_{\varepsilon}^{(n)}=\varepsilon \sum_{i=1}^{n}-\Delta_{x_{i}}+\varepsilon^{2} \sum_{1 \leq i<j \leq n} V\left(x_{i}-x_{j}\right),
$$

where $\varepsilon$ is a positive parameter and $x_{i}, x_{j} \in \mathbb{R}^{d}$. We assume that the particles obey Bose statistics. So, we consider $H_{\varepsilon}^{(n)}$ as an operator acting on the space $L_{s}^{2}\left(\mathbb{R}^{d n}\right)$ of symmetric square-integrable functions. This means that

$\Psi \in L_{s}^{2}\left(\mathbb{R}^{d n}\right)$ if and only if $\Psi \in L^{2}\left(\mathbb{R}^{d n}\right)$ and $\Psi\left(x_{1}, \cdots, x_{n}\right)=\Psi\left(x_{\sigma_{1}}, \ldots, x_{\sigma_{n}}\right)$ a.e.

for any permutation $\sigma$ in the symmetric group $\mathfrak{S}_{n}$. The mean field asymptotics is concerned with the limit as $\varepsilon \rightarrow 0$ and $n \varepsilon \rightarrow 1$, where $n=\left[\frac{1}{\varepsilon}\right]$ represents the number of particles of the system. 
Let $\mathcal{H}$ be a direct sum of Hilbert spaces of the form

$$
\mathcal{H}=\bigoplus_{n=0}^{\infty} L_{s}^{2}\left(\mathbb{R}^{d n}\right)
$$

and consider the Hamiltonian of the many-bosons system (with arbitrary number of particles) as

$$
H_{\varepsilon}=\bigoplus_{n=0}^{\infty} H_{\varepsilon}^{(n)}
$$

An obvious feature of the operator $H_{\varepsilon}$ is the conservation of the number of particles. Hence, it is useful to define the number operator

$$
\mathbf{N}=\bigoplus_{n=0}^{\infty} \varepsilon n \mathbb{1}_{L_{S}^{2}\left(\mathbb{R}^{d n}\right)} .
$$

The free Hamiltonian, corresponding to $V=0$, will be denoted by $H_{\varepsilon}^{0}$ :

$$
H_{\varepsilon}^{0}=\bigoplus_{n=0}^{\infty} H_{\varepsilon}^{0,(n)}, \quad H_{\varepsilon}^{0,(n)}=\varepsilon \sum_{i=1}^{n}-\Delta_{x_{i}} .
$$

Second quantization is a natural framework for the study of many-body problems and, even more, it helps to understand the mean field limit and the structures behind it. However, the result can be presented without using the language of quantum field theory. We just mention that the operator $H_{\varepsilon}$ can be formally rewritten as

$$
H_{\varepsilon}=\int_{\mathbb{R}^{d}} \nabla a^{*}(x) \cdot \nabla a(x) d x+\frac{1}{2} \int_{\mathbb{R}^{2 d}} V(x-y) a^{*}(x) a^{*}(y) a(x) a(y) d x d y,
$$

with the $\varepsilon$-dependent canonical commutation relations $\left[a(x), a^{*}(y)\right]=\varepsilon \delta(x-y)$. It is interpreted as the Wick quantization of the classical Hamiltonian

$$
h(z, \bar{z})=\int_{\mathbb{R}^{d}}|\nabla z(x)|^{2} d x+\frac{1}{2} \int_{\mathbb{R}^{2 d}}|z(x)|^{2}|z(y)|^{2} V(x-y) d x d y .
$$

In our analysis, an operator which violates the number of particles conservation will play an important role, namely the Weyl operator. Such operators are given for $f \in L^{2}\left(\mathbb{R}^{d}\right)$ by

$$
W(f)=e^{\frac{i}{\sqrt{2}}\left[a^{*}(f)+a(f)\right]},
$$

where $a^{*}(f), a(f)$ are the creation-annihilation operators on $\mathcal{H}$ satisfying the $\varepsilon$ canonical commutation relations (CCR):

$$
\left[a\left(f_{1}\right), a^{*}\left(f_{2}\right)\right]=\varepsilon\left\langle f_{1}, f_{2}\right\rangle_{L^{2}\left(\mathbb{R}^{d}\right)} \mathbb{1}, \quad\left[a^{*}\left(f_{1}\right), a^{*}\left(f_{2}\right)\right]=0=\left[a\left(f_{1}\right), a\left(f_{2}\right)\right] .
$$

Accurate definitions on second quantized operators can be found in Appendix B. 
Our approach is based on Wigner measures, which are Borel probability measures on the infinite-dimensional phase-space $\mathcal{Z}_{0}:=L^{2}\left(\mathbb{R}^{d} ; \mathbb{C}\right)$. The states of the many-bosons system are positive trace-class operators on $\mathcal{H}$ of normalized trace equal to 1 (i.e. normal states or density operators). To every family of such states $\left(\varrho_{\varepsilon}\right)_{\varepsilon \in(0, \bar{\varepsilon})}$ we asymptotically assign, when $\varepsilon \rightarrow 0$, at least one Borel probability measure $\mu$ on $\mathcal{Z}_{0}:=L^{2}\left(\mathbb{R}^{d} ; \mathbb{C}\right)$, called Wigner measure, such that there exists a sequence $\left(\varepsilon_{k}\right)_{k \in \mathbb{N}}$ such that $\lim _{k \rightarrow \infty} \varepsilon_{k}=0$ and

$$
\lim _{k \rightarrow 0} \operatorname{Tr}\left[\varrho_{\varepsilon_{k}} W(\sqrt{2} \pi \xi)\right]=\mathcal{F}^{-1}(\mu)(\xi)
$$

under the sole uniform estimate $\operatorname{Tr}\left[\varrho_{\varepsilon} \mathbf{N}^{\delta}\right] \leq C_{\delta}$ for some $\delta>0$. Here $\mathcal{F}^{-1}(\mu)$ is the inverse Fourier transform of $\mu$.

The problem of the mean field dynamics questions whether the asymptotic quantities, namely Wigner measures, as $\varepsilon \rightarrow 0$ associated with

$$
\varrho_{\varepsilon}(t)=e^{-i \frac{t}{\varepsilon} H_{\varepsilon}} \varrho_{\varepsilon} e^{i \frac{t}{\varepsilon} H_{\varepsilon}}, \quad t \in \mathbb{R}
$$

are transported by the flow $\Phi(t, s)=\Phi(t-s)$ generated by the classical Hamiltonian $h(z, \bar{z})$ and given, after writing $z_{t}=\Phi(t, s)\left(z_{s}\right)$, by

$$
i \partial_{t} z_{t}=\left(\partial_{\bar{z}} h\right)\left(z_{t}, \bar{z}_{t}\right)=-\Delta z_{t}+V *\left|z_{t}\right|^{2} z_{t} .
$$

After checking that the Hamiltonian (1.1) has a self-adjoint realization so that the quantum dynamics are well-defined on $\mathcal{H}$ and after checking that the mean field flow is well defined on $\mathcal{Z}_{1}=H^{1}\left(\mathbb{R}^{d}\right)$, our main result is stated below.

Throughout the paper, we assume that the real valued potential $V$ satisfies the assumptions

$$
\begin{aligned}
& \quad V(-x)=V(x) \in \mathbb{R}, \\
& V(1-\Delta)^{-1 / 2} \in \mathcal{L}\left(\mathcal{Z}_{0}\right), \\
& \text { and } \quad(1-\Delta)^{-1 / 2} V(1-\Delta)^{-1 / 2} \in \mathcal{L}^{\infty}\left(\mathcal{Z}_{0}\right) .
\end{aligned}
$$

We use the notation $\mathcal{L}(\mathfrak{h})$ for the space of bounded operators on the Hilbert space $\mathfrak{h}$ and $\mathcal{L}^{p}(\mathfrak{h}), 1 \leq p \leq+\infty$, for the Schatten classes, $\mathcal{L}^{\infty}(\mathfrak{h})$ being the space of compact operators for $p=+\infty$.

Theorem 1.1. Let $\left(\varrho_{\varepsilon}\right)_{\varepsilon \in(0, \bar{\varepsilon})}$ be a family of normal states on $\mathcal{H}$ with a single Wigner measure $\mu_{0}$ such that the bound

$$
\operatorname{Tr}\left[\left(\mathbf{N}+H_{\varepsilon}^{0}\right)^{\delta} \varrho_{\varepsilon}\right] \leq C_{\delta}<+\infty,
$$

holds uniformly with respect to $\varepsilon \in(0, \bar{\varepsilon})$ for some $\delta>0$. Then for all $t \in \mathbb{R}$, the family $\left(e^{-i \frac{t}{\varepsilon} H_{\varepsilon}} \varrho_{\varepsilon} e^{i \frac{t}{\varepsilon} H_{\varepsilon}}\right)_{\varepsilon \in(0, \bar{\varepsilon})}$ has a unique Wigner measure $\mu_{t}$ which is a Borel measure on $\mathcal{Z}_{1}=H^{1}\left(\mathbb{R}^{d}\right)$. This measure $\mu_{t}=\Phi(t, 0)_{*} \mu_{0}$ is the push forward of the initial measure $\mu_{0}$ by the flow associated with (1.3), well-defined on $\mathcal{Z}_{1}$. 
At a formal level the proof of the above theorem is rather simple. First write the integral formula

$$
\operatorname{Tr}\left[\varrho_{\varepsilon}(t) W(\xi)\right]=\operatorname{Tr}\left[\varrho_{\varepsilon} W(\xi)\right]+i \int_{0}^{t} \operatorname{Tr}\left[\varrho_{\varepsilon}(s) W(\xi) \sum_{j=1}^{4} \varepsilon^{j-1} \mathcal{O}_{j}\right] d s
$$

where $\varrho_{\varepsilon}(t)=e^{-i t / \varepsilon H_{\varepsilon}} \varrho_{\varepsilon} e^{i t / \varepsilon H_{\varepsilon}}$ and $\mathcal{O}_{j}$ are some Wick quantized observables. By taking the limit as $\varepsilon \rightarrow 0$, only the term $j=1$ is left in the right-hand side. So, we formally end up with a transport equation on the Wigner measures

$$
\partial_{t} \mu+i\{h, \mu\}=0, \quad\{h, \mu\}=\partial_{z} h \partial_{\bar{z}} \mu-\partial_{z} \mu \partial_{\bar{z}} h
$$

which is then solved by appealing to the results in [3].

Outline: The self-adjointness of the Hamiltonian $H_{\varepsilon}$ and the existence of a global flow on $\mathcal{Z}_{1}=H^{1}\left(\mathbb{R}^{d}\right)$ for the Hartree equation (1.3) are proved in Section 2. The derivation of the mean field dynamics is done in Section 3, where Theorem 1.1 is proved. Some additional properties are stated in Section 4: in particular, we draw the link with former results on bounded potential and reduced density matrices and provide non-trivial examples elucidated by the Wigner measure approach. The article ends with several appendices dedicated to second quantization, absolutely continuous curves in $\operatorname{Prob}_{2}(\mathcal{Z})$ as well as some weak $L^{p}$ conditions for the potential $V$ ensuring the fulfillment of the assumptions (A2) and (A3).

\section{Well-defined dynamics}

In this section we shall prove that:

- the quantum dynamics is well-defined, namely $H_{\varepsilon}$ has a natural self-adjoint realization;

- the mean field dynamics is well-defined on $\mathcal{Z}_{1}=H^{1}\left(\mathbb{R}^{d}\right)$, with additional useful estimates.

\subsection{Self-adjoint realization of $H_{\varepsilon}$}

The Hamiltonian $H_{\varepsilon}$ has a particular structure explained in a general framework in Appendix A.

Let $V$ be a real-valued Lebesgue measurable function a.e. finite and satisfying the assumptions (A1) and (A2). The multiplication operator

$$
V_{\varepsilon}^{(n)}=\varepsilon^{2} \sum_{1 \leq i<j \leq n} V\left(x_{i}-x_{j}\right)
$$


with its natural domain $\mathcal{D}\left(V_{\varepsilon}^{(n)}\right)=\left\{\Psi \in L_{s}^{2}\left(\mathbb{R}^{d n}\right): V_{\varepsilon}^{(n)} \Psi \in L_{s}^{2}\left(\mathbb{R}^{d n}\right)\right\}$ is selfadjoint on $L_{s}^{2}\left(\mathbb{R}^{d n}\right)$ as well as the differential operator

$$
H_{\varepsilon}^{0,(n)}=\varepsilon \sum_{i=1}^{n}-\Delta_{x_{i}}, \quad \text { with } \quad \mathcal{D}\left(H_{\varepsilon}^{0,(n)}\right)=L_{s}^{2}\left(\mathbb{R}^{d n}\right) \cap H^{2}\left(\mathbb{R}^{d n}\right) .
$$

Therefore, according to Appendix A

$$
V_{\varepsilon}=\sum_{n=0}^{\infty} V_{\varepsilon}^{(n)}, \quad \text { and } \quad H_{\varepsilon}^{0}=\sum_{n=0}^{\infty} H_{\varepsilon}^{0,(n)}
$$

endowed with their natural domains are self-adjoint on $\mathcal{H}$.

Proposition 2.1. Under the assumptions (A1) and (A2):

(i) The operator $H_{\varepsilon}^{(n)}:=H_{\varepsilon}^{0,(n)}+V_{\varepsilon}^{(n)}$ is self-adjoint on $\mathcal{D}\left(H_{\varepsilon}^{0,(n)}\right) \subset \mathcal{D}\left(V_{\varepsilon}^{(n)}\right)$;

(ii) The operator

$$
H_{\varepsilon}:=\sum_{n=0}^{\infty} H_{\varepsilon}^{(n)}, \quad \mathcal{D}\left(H_{\varepsilon}\right):=\left\{\Psi \in \mathcal{H}, \sum_{n=0}^{\infty}\left\|H_{\varepsilon}^{(n)} \Psi^{(n)}\right\|^{2}<\infty\right\},
$$

is self-adjoint and essentially self-adjoint on $\oplus_{n \in \mathbb{N}}^{\text {alg }} \mathcal{D}_{n}$, where $\mathcal{D}_{n}$ is any core of $H_{\varepsilon}^{0,(n)}$.

Proof. (i) By assumption (A2), $V_{\varepsilon}^{(n)}$ is infinitesimally small with respect to $H_{\varepsilon}^{0,(n)}$. So that, $\mathcal{D}\left(H_{\varepsilon}^{0,(n)}\right) \subset \mathcal{D}\left(V_{\varepsilon}^{(n)}\right)$ and the operator $H_{\varepsilon}^{(n)}=H_{\varepsilon}^{0,(n)}+V_{\varepsilon}^{(n)}$ is self-adjoint on the domain of $H_{\varepsilon}^{0,(n)}$ by the Kato-Rellich theorem.

(ii) Applying Proposition A.1, we see that $H_{\varepsilon}$ is self-adjoint and essentially selfadjoint on $\oplus_{n \in \mathbb{N}}^{\text {alg }} \mathcal{D}_{n}$.

Later, it will be useful to use the reference operator

$$
S_{\varepsilon}(\lambda)=\sum_{n=0}^{\infty} H_{\varepsilon}^{0,(n)}+\varepsilon n+\lambda(\varepsilon n)^{3}
$$

which is self-adjoint by Proposition A.1. Moreover, by functional calculus of strongly commuting self-adjoint operators we observe that $\mathcal{D}\left(S_{\varepsilon}(\lambda)\right)$ is invariant with respect to the parameter $\lambda>0$.

Proposition 2.2. Under the assumptions (A1) and (A2), for any $\lambda>0$, the operator $V_{\varepsilon}$ is $S_{\varepsilon}(\lambda)$-bounded with

$$
\forall \Psi \in \mathcal{D}\left(S_{\varepsilon}(\lambda)\right), \quad\left\|V_{\varepsilon} \Psi\right\|_{\mathcal{H}} \leq \lambda\left\|V(1-\Delta)^{-1 / 2}\right\|_{\mathcal{L}\left(L^{2}\left(\mathbb{R}^{d}\right)\right)}\left\|S_{\varepsilon}\left(\lambda^{-2}\right) \Psi\right\|_{\mathcal{H}} .
$$

Therefore $H_{\varepsilon}$ is essentially self-adjoint on $\mathcal{D}\left(S_{\varepsilon}(\lambda)\right)$. 
Proof. The multiplication operator by $V\left(x_{1}-x_{2}\right)$, at least defined as a symmetric operator from $\mathcal{S}\left(\mathbb{R}^{2 d}\right)$ into $\mathcal{S}^{\prime}\left(\mathbb{R}^{2 d}\right)$, satisfies

$$
\begin{aligned}
& e^{i x_{2} D_{x_{1}} V\left(x_{1}-x_{2}\right)\left(1-\Delta_{x_{1}}\right)^{-1 / 2} e^{-i x_{2} D_{x_{1}}}} \\
& =e^{i x_{2} D_{x_{1}}} V\left(x_{1}-x_{2}\right) e^{-i x_{2} D_{x_{1}}}\left(1-\Delta_{x_{1}}\right)^{-1 / 2} \\
& =V\left(x_{1}\right)\left(1-\Delta_{x_{1}}\right)^{-1 / 2} \in \mathcal{L}\left(L^{2}\left(\mathbb{R}^{2 d}\right)\right) \text {. }
\end{aligned}
$$

For $\Psi \in \mathcal{H}, \Phi \in \mathcal{D}\left(S_{\varepsilon}(\lambda)\right)$, taking advantage of the symmetry of those wave functions, we compute

$$
\begin{aligned}
\left\langle\Psi, V_{\varepsilon} \Phi\right\rangle & =\sum_{n=2}^{\infty}\left\langle\Psi^{(n)}, \frac{n(n-1)}{2} \varepsilon^{2} V\left(x_{1}-x_{2}\right) \Phi^{(n)}\right\rangle_{L_{s}^{2}\left(\mathbb{R}^{d n}\right)} \\
& =\sum_{n=2}^{\infty}\left\langle\Psi^{(n)}, \frac{n(n-1)}{2} \varepsilon^{2} V\left(x_{1}-x_{2}\right)\left(1-\Delta_{x_{1}}\right)^{-1 / 2}\left(1-\Delta_{x_{1}}\right)^{1 / 2} \Phi^{(n)}\right\rangle_{L_{s}^{2}\left(\mathbb{R}^{d n}\right)} .
\end{aligned}
$$

By noticing that

$$
\begin{aligned}
\left(n^{2} \varepsilon^{2}\right)^{2}\left\|\left(1-\Delta_{x_{1}}\right)^{1 / 2} \Phi^{(n)}\right\|_{L^{2}\left(\mathbb{R}^{n d}\right)}^{2} & =(\varepsilon n)^{3}\left\langle\Phi^{(n)}, \varepsilon \sum_{i=1}^{n}\left(1-\Delta_{x_{i}}\right) \Phi^{(n)}\right\rangle_{L_{s}^{2}\left(\mathbb{R}^{d n}\right)} \\
& =\left\|\mathbf{N}^{3 / 2}\left(\mathbf{N}+H_{\varepsilon}^{0}\right)^{1 / 2} \Phi^{(n)}\right\|_{\mathcal{H}}^{2},
\end{aligned}
$$

the Cauchy-Schwarz inequality leads to

$$
\left|\left\langle\Psi, \quad V_{\varepsilon} \Phi\right\rangle\right| \leq\left\|V(1-\Delta)^{-1 / 2}\right\|\|\Psi\|_{\mathcal{H}}\left\|\mathbf{N}^{3 / 2}\left(\mathbf{N}+H_{\varepsilon}^{0}\right)^{1 / 2} \Phi\right\|_{\mathcal{H}}
$$

Now with the inequality $a b \leq(\lambda a)^{2}+(b / \lambda)^{2}$, we see that

$$
\begin{aligned}
\left\|\mathbf{N}^{3 / 2}\left(\mathbf{N}+H_{\varepsilon}^{0}\right)^{1 / 2} \Phi\right\|_{\mathcal{H}}^{2} & =\left\langle\Phi, \mathbf{N}^{3}\left(\mathbf{N}+H_{\varepsilon}^{0}\right) \Phi\right\rangle \\
& \leq\left\langle\Phi, \lambda^{-2} \mathbf{N}^{6}+\lambda^{2}\left(\mathbf{N}+H_{\varepsilon}^{0}\right)^{2} \Phi\right\rangle \\
& \leq \lambda^{2}\left\|S_{\varepsilon}\left(\lambda^{-2}\right) \Phi\right\|_{\mathcal{H}}^{2} .
\end{aligned}
$$

Putting together (2.3) and (2.5) yields the estimate.

To prove the last statement, observe that $\oplus_{n \in \mathbb{N}}^{\mathrm{alg}} \mathcal{D}\left(H_{\varepsilon}^{0,(n)}\right)$ is a core for $H_{\varepsilon}$. Owing to the inclusions

$$
\oplus_{n \in \mathbb{N}}^{\mathrm{alg}} \mathcal{D}\left(H_{\varepsilon}^{0,(n)}\right) \subset \mathcal{D}\left(S_{\varepsilon}(\lambda)\right) \subset \mathcal{D}\left(V_{\varepsilon}\right) \cap \mathcal{D}\left(H_{\varepsilon}^{0}\right) \subset \mathcal{D}\left(H_{\epsilon}\right),
$$

$H_{\varepsilon}$ is essentially self-adjoint on $\mathcal{D}\left(S_{\varepsilon}(\lambda)\right)$.

We end this section with some invariance properties of the domain $\mathcal{D}\left(S_{\varepsilon}(\lambda)\right)$ with respect to the Hamiltonian $H_{\varepsilon}$ and the Weyl operators. 
Proposition 2.3. For any $\lambda>0$ and $t \in \mathbb{R}$

$$
e^{-i \frac{t}{\varepsilon} H_{\varepsilon}} \mathcal{D}\left(S_{\varepsilon}(\lambda)\right) \subset \mathcal{D}\left(S_{\varepsilon}(\lambda)\right)
$$

Moreover there exists $C_{\lambda}>0$ such that

$$
\left\|S_{\varepsilon}(\lambda) e^{-i \frac{t}{\varepsilon} H_{\varepsilon}}\left(S_{\varepsilon}(\lambda)+1\right)^{-1}\right\|_{\mathcal{L}(\mathcal{H})} \leq C_{\lambda}, \quad \text { for all } t \in \mathbb{R}
$$

Proof. For any $\Psi \in \mathcal{D}\left(S_{\varepsilon}(\lambda)\right) \subset \mathcal{D}\left(H_{\varepsilon}\right)$, observe that $e^{-i \frac{t}{\varepsilon} H_{\varepsilon}} \Psi$ belongs to $\mathcal{D}\left(\mathbf{N}^{3}\right) \cap$ $\mathcal{D}\left(H_{\varepsilon}\right)$ since $\mathcal{D}\left(S_{\varepsilon}(\lambda)\right)$ is contained in $\mathcal{D}\left(\mathbf{N}^{3}\right)$ and $H_{\varepsilon}$ strongly commutes with $\mathbf{N}$. Proposition 2.2 implies $\mathcal{D}\left(S_{\varepsilon}(\beta)+V_{\varepsilon}\right)=\mathcal{D}\left(S_{\varepsilon}(\beta)\right)=\mathcal{D}\left(S_{\varepsilon}(\lambda)\right)$ when $\beta>0$ is large enough, so that for any $\Phi \in \mathcal{D}\left(S_{\varepsilon}(\lambda)\right)$

$$
\begin{aligned}
\left\langle\left(S_{\varepsilon}(\beta)+V_{\varepsilon}\right) \Phi, e^{-i \frac{t}{\varepsilon} H_{\varepsilon}} \Psi\right\rangle_{\mathcal{H}} & =\left\langle\left(H_{\varepsilon}+\mathbf{N}+\beta \mathbf{N}^{3}\right) \Phi, e^{-i \frac{t}{\varepsilon} H_{\varepsilon}} \Psi\right\rangle_{\mathcal{H}} \\
& =\left\langle\Phi,\left(H_{\varepsilon}+\mathbf{N}+\beta \mathbf{N}^{3}\right) e^{-i \frac{t}{\varepsilon} H_{\varepsilon}} \Psi\right\rangle_{\mathcal{H}}
\end{aligned}
$$

and hence $e^{-i \frac{t}{\varepsilon} H_{\varepsilon}} \Psi$ belongs to $\mathcal{D}\left(\left(S_{\varepsilon}(\beta)+V_{\varepsilon}\right)^{*}\right)=\mathcal{D}\left(S_{\varepsilon}(\beta)\right)=\mathcal{D}\left(S_{\varepsilon}(\lambda)\right)$.

Again for $\beta$ large enough

$$
1+S_{\varepsilon}(\beta)+V_{\varepsilon}=\left(1+V_{\varepsilon}\left(S_{\varepsilon}(\beta)+1\right)^{-1}\right)\left(S_{\varepsilon}(\beta)+1\right),
$$

and

$$
\left(1+\mathbf{N}+\beta \mathbf{N}^{3}+H_{\varepsilon}\right)^{-1}=\left(S_{\varepsilon}(\beta)+1\right)^{-1}\left(1+V_{\varepsilon}\left(S_{\varepsilon}(\beta)+1\right)^{-1}\right)^{-1} .
$$

Therefore, the operators $\left(1+S_{\varepsilon}(\beta)\right)\left(1+\mathbf{N}+\beta \mathbf{N}^{3}+H_{\varepsilon}\right)^{-1}$ and $\left(1+\mathbf{N}+\beta \mathbf{N}^{3}+\right.$ $\left.H_{\varepsilon}\right)\left(S_{\varepsilon}(\beta)+1\right)^{-1}$ are bounded. Thus, we conclude that

$$
\begin{gathered}
S_{\varepsilon}(\lambda) e^{-i \frac{t}{\varepsilon} H_{\varepsilon}}\left(1+S_{\varepsilon}(\lambda)\right)^{-1}=S_{\varepsilon}(\lambda)\left(1+S_{\varepsilon}(\beta)\right)^{-1}\left(1+S_{\varepsilon}(\beta)\right)\left(1+\mathbf{N}+\beta \mathbf{N}^{3}+H_{\varepsilon}\right)^{-1} \\
\circ e^{-i \frac{t}{\varepsilon} H_{\varepsilon}}\left(1+\mathbf{N}+\beta \mathbf{N}^{3}+H_{\varepsilon}\right)\left(1+S_{\varepsilon}(\beta)\right)^{-1}\left(1+S_{\varepsilon}(\beta)\right)\left(1+S_{\varepsilon}(\lambda)\right)^{-1}
\end{gathered}
$$

is bounded.

Proposition 2.4. For any $\xi \in H^{2}\left(\mathbb{R}^{d}\right)$ and any $\lambda>0$, the domain $\mathcal{D}\left(S_{\varepsilon}(\lambda)\right)$ is invariant under the action of the Weyl operator $W(\xi)$ with

$$
\left\|\left(S_{\varepsilon}(\lambda)+1\right)^{-1} W(\xi) S_{\varepsilon}(\lambda)\right\|_{\mathcal{L}(\mathcal{H})} \leq C_{\lambda, \xi},
$$

uniformly with respect to $\varepsilon \in(0, \bar{\varepsilon})$ for some constant $C_{\lambda, \xi}>0$.

Proof. For all $\Phi, \Psi \in \mathcal{D}\left(S_{\varepsilon}(\lambda)\right)$, one can write

$$
\left\langle\Phi, W(\xi)^{*} S_{\varepsilon}(\lambda) W(\xi) \Psi\right\rangle=\left\langle\Phi,\left(S_{\varepsilon}(\lambda)+Q_{\varepsilon}^{\text {Wick }}\right) \Psi\right\rangle,
$$


where $Q_{\varepsilon}$ is the polynomial

$Q_{\varepsilon}(z)=\left\langle z+\frac{i \varepsilon}{\sqrt{2}} \xi,-\Delta\left(z+\frac{i \varepsilon}{\sqrt{2}} \xi\right)\right\rangle_{\mathcal{Z}_{0}}-\langle z,-\Delta z\rangle_{\mathcal{Z}_{0}}+P_{\varepsilon}\left(z+\frac{i \varepsilon}{\sqrt{2}} \xi\right)-P_{\varepsilon}(z)$

and $P_{\varepsilon}(z)=|z|_{\mathcal{Z}_{0}}^{6}+3 \varepsilon|z|_{\mathcal{Z}_{0}}^{4}+\varepsilon^{2}|z|_{\mathcal{Z}_{0}}^{2}$ is the complete Wick symbol of $\mathbf{N}^{3}$, according to Proposition B.2 or by direct computation. The assumption $\xi \in H^{2}\left(\mathbb{R}^{d}\right)$ ensures that $Q_{\varepsilon}$ is uniformly bounded in $\oplus_{p+q \leq 3} \mathcal{P}_{p, q}\left(\mathcal{Z}_{0}\right)$ and the number estimate of Proposition B.3 says that $Q_{\varepsilon}^{\text {Wick }}\langle\mathbf{N}\rangle^{-\frac{3}{2}}$ is a bounded operator and therefore

$$
Q_{\varepsilon}^{\text {Wick }}\left(S_{\varepsilon}(\lambda)+1\right)^{-1} \in \mathcal{L}(\mathcal{H}) .
$$

Hence for $\Psi \in \mathcal{D}\left(S_{\varepsilon}(\lambda)\right)$,

$$
S_{\varepsilon}(\lambda) W(\xi) \Psi=W(\xi)\left[\frac{S_{\varepsilon}(\lambda)}{S_{\varepsilon}(\lambda)+1}+Q_{\varepsilon}^{\text {Wick }}\left(S_{\varepsilon}(\lambda)+1\right)^{-1}\right]\left(S_{\varepsilon}(\lambda)+1\right) \Psi
$$

and $W(\xi) \Psi$ belongs to $\mathcal{D}\left(S_{\varepsilon}(\lambda)\right)$, with $\left\|S_{\varepsilon}(\lambda) W(\xi) \Psi\right\| \leq C_{\lambda, \xi}\left\|\left(S_{\varepsilon}(\lambda)+1\right) \Psi\right\|$.

Proposition 2.5. For any function $\chi \in \mathcal{C}_{0}^{\infty}\left(\mathbb{R}^{2}\right)$ and $\lambda>0$, the operator $\chi\left(\mathbf{N}, H_{\varepsilon}\right)$ satisfies

$$
\forall k \in \mathbb{N}, \quad\left\|\mathbf{N}^{k} S_{\varepsilon}(\lambda) \chi\left(\mathbf{N}, H_{\varepsilon}\right)\right\|_{\mathcal{L}(\mathcal{H})} \leq C_{\lambda, \chi}^{k+1}
$$

for some $C_{\lambda, \chi}>0$.

Proof. The operators $\mathbf{N}, H_{\varepsilon}$ (like $\mathbf{N}$ and $\mathbf{N}+H_{\varepsilon}^{0}$ ) are strongly commuting selfadjoint operators so that the functional calculus is well defined for the pair $\left(\mathbf{N}, H_{\varepsilon}\right)$. With a cut-off function $\chi_{1} \in \mathcal{C}_{0}^{\infty}(\mathbb{R})$ such that $\chi_{1}(x) \equiv 1$ on a neighborhood of supp $\chi$, the operator $\mathbf{N}^{k}\left(1+\mathbf{N}+\beta \mathbf{N}^{3}+H_{\varepsilon}\right) \chi_{1}(\mathbf{N}) \chi\left(H_{\varepsilon}, \mathbf{N}\right)$ is bounded with

$$
\left\|\left(1+\mathbf{N}+\beta \mathbf{N}^{3}+H_{\varepsilon}\right) \mathbf{N}^{k} \chi_{1}(\mathbf{N}) \chi(H, \mathbf{N})\right\|_{\mathcal{L}(\mathcal{H})} \leq C_{\beta} C_{\chi}^{k} .
$$

For sufficiently large $\beta$, Proposition 2.2 says

$$
\left\|\left(1+S_{\varepsilon}(\beta)\right)\left(1+\mathbf{N}+\beta \mathbf{N}^{3}+H_{\varepsilon}\right)^{-1}\right\|_{\mathcal{L}(\mathcal{H})} \leq C_{\beta}^{\prime} .
$$

This is done with

$$
\begin{aligned}
\mathbf{N}^{k} S_{\varepsilon}(\lambda) \chi\left(\mathbf{N}, H_{\varepsilon}\right)=S_{\varepsilon}(\lambda)\left(1+S_{\varepsilon}(\beta)\right)^{-1} & \left(1+S_{\varepsilon}(\beta)\left(1+\mathbf{N}+\beta \mathbf{N}^{3}+H_{\varepsilon}\right)^{-1}\right. \\
& \circ\left(1+\mathbf{N}+\beta \mathbf{N}^{3}+H_{\varepsilon}\right) \mathbf{N}^{k} \chi\left(\mathbf{N}, H_{\varepsilon}\right),
\end{aligned}
$$

and $C_{\lambda, \chi}=\max \left\{C_{\chi}: C_{\beta} C_{\beta}^{\prime}\left\|S_{\varepsilon}(\lambda)\left(1+S_{\varepsilon}(\beta)\right)^{-1}\right\|\right\}$. 


\subsubsection{Mean field dynamics}

We shall use another more convenient writing of the Cauchy problem

$$
\left\{\begin{array}{l}
i \partial_{t} z_{t}=-\Delta z_{t}+V *\left|z_{t}\right|^{2} z_{t} \\
z_{t=0}=z_{0}
\end{array}\right.
$$

After setting $\tilde{z}_{t}=e^{i t(-\Delta)} z_{t}=e^{-i t \Delta} z_{t}$ it becomes

$$
\left\{\begin{array}{l}
i \partial_{t} \tilde{z}_{t}=e^{-i t \Delta}\left[V *\left|e^{i t \Delta} \tilde{z}_{t}\right|^{2}\left(e^{i t \Delta} \tilde{z}_{t}\right)\right] \\
\tilde{z}_{t=0}=z_{0} .
\end{array}\right.
$$

Proposition 2.6. Assume (A1) and (A2). For any $z_{0} \in \mathcal{Z}_{1}=H^{1}\left(\mathbb{R}^{d}\right)$ the Cauchy problem (2.5) admits a unique solution $\left(t \mapsto z_{t}\right) \in \mathcal{C}^{0}\left(\mathbb{R} ; H^{1}\left(\mathbb{R}^{d}\right)\right) \cap \mathcal{C}^{1}\left(\mathbb{R} ; H^{-1}\left(\mathbb{R}^{d}\right)\right)$. More precisely, the Cauchy problem (2.6), which is equivalent to (2.5), admits a unique solution in $\mathcal{C}^{1}\left(\mathbb{R} ; H^{1}\left(\mathbb{R}^{d}\right)\right)$. Moreover these solutions verify

$$
\begin{aligned}
& \quad\left|z_{t}\right|_{L^{2}}=\left|\tilde{z}_{t}\right|_{L^{2}}=\left|z_{0}\right|_{L^{2}} \\
& \text { and } h\left(z_{t}, \overline{z_{t}}\right)=h\left(z_{0}, \overline{z_{0}}\right) \\
& \text { for } h(z, \bar{z})=\int_{\mathbb{R}^{d}}|\nabla z|^{2}(x) d x+\frac{1}{2} \int_{\mathbb{R}^{2 d}} V(x-y)|z(x)|^{2}|z(y)|^{2} d x d y .
\end{aligned}
$$

Finally, the time-dependent velocity field defined on $\mathbb{R} \times \mathcal{Z}_{1}$ by

$$
v(t, z)=e^{-i t \Delta}\left(\left[V *\left|e^{i t \Delta} z\right|^{2}\right] e^{i t \Delta} z\right)
$$

satisfies the estimates

$$
\begin{aligned}
|v(t, z)|_{\mathcal{Z}_{0}} & \leq\left\|V(1-\Delta)^{-1 / 2}\right\||z|_{\mathcal{Z}_{0}}^{2}|z| \mathcal{Z}_{1} \\
\text { and } \quad|v(t, z)|_{\mathcal{Z}_{1}} & \leq\left\|V(1-\Delta)^{-1 / 2}\right\||z|_{\mathcal{Z}_{1}}^{2}|z| \mathcal{Z}_{0}
\end{aligned}
$$

Proof. The first results are standard (see e.g. $[16,31]$ ) in the analysis on nonlinear evolution equation. Nevertheless, we recall the details of the proof because it also contains (2.9)(2.10), which is crucial in our analysis.

By considering the second formulation (2.6), it suffices to prove that the mapping $z \rightarrow\left(V *|z|^{2}\right) z$ is locally Lipschitz in $H^{1}\left(\mathbb{R}^{d}\right)$. After noticing that the distributional derivative of $\left(V *|z|^{2}\right) z$ or more generally of $\left(V *\left(\overline{z_{1}} z_{2}\right) z_{3}\right)$ is

$$
\partial_{x}\left[\left(V *\left(\overline{z_{1}} z_{2}\right)\right) z_{3}\right]=\left(V *\left(\partial_{x} \bar{z}_{1} z_{2}+\bar{z}_{1} \partial_{x} z_{2}\right)\right) z_{3}+\left(V *\left(\bar{z}_{1} z_{2}\right)\right)\left(\partial_{x} z_{3}\right)
$$

it is reduced to the estimate of $V *\left(\overline{z_{1}} z_{2}\right) z_{3}$ in $L^{2}$ in terms of the $L^{2}$ and $H^{1}$ - norms of $z_{1}, z_{2}, z_{3}$. For $\xi \in L^{2}\left(\mathbb{R}^{d}\right)$, write

$$
\left\langle\xi,\left(V *\left(\overline{z_{1}} z_{2}\right)\right) z_{3}\right\rangle_{L^{2}\left(\mathbb{R}^{d}\right)}=\left\langle z_{1} \otimes \xi, V\left(x_{1}-x_{2}\right) z_{2} \otimes z_{3}\right\rangle_{L^{2}\left(\mathbb{R}^{2 d}\right)}
$$


When $\tilde{b}$ is the multiplication operator by $V\left(x_{1}-x_{2}\right)$, the estimate (2.2) says that $\tilde{b}\left(1-\Delta_{x_{1}}\right)^{-1 / 2}$ is bounded, with

$\left|\left\langle\xi \otimes z_{1}, V\left(x_{1}-x_{2}\right) z_{2} \otimes z_{3}\right\rangle_{L^{2}\left(\mathbb{R}^{2 d}\right)}\right| \leq\left\|V(1-\Delta)^{-1 / 2}\right\||\xi|_{L^{2}}\left|z_{1}\right|_{L^{2}}\left|z_{2}\right|_{H^{1}}\left|z_{3}\right|_{L^{2}}$.

A symmetric version of (2.2) says $\tilde{b}\left(1-\Delta_{x_{2}}\right)^{-1 / 2}$ is bounded, with

$\left|\left\langle\xi \otimes z_{1}, V\left(x_{1}-x_{2}\right) z_{2} \otimes z_{3}\right\rangle_{L^{2}\left(\mathbb{R}^{2 d}\right)}\right| \leq\left\|V(1-\Delta)^{-1 / 2}\right\||\xi|_{L^{2}}\left|z_{1}\right|_{L^{2}}\left|z_{2}\right|_{L^{2}}\left|z_{3}\right|_{H^{1}}$.

Finally the symmetry of the expression $V *\left(\overline{z_{1}} z_{2}\right) z_{3}$ with respect to the exchange of $\overline{z_{1}}$ and $z_{2}$ gives

$\left|\left\langle\xi \otimes z_{1}, V\left(x_{1}-x_{2}\right) z_{2} \otimes z_{3}\right\rangle_{L^{2}\left(\mathbb{R}^{2 d}\right)}\right| \leq\left\|V(1-\Delta)^{-1 / 2}\right\||\xi|_{L^{2}}\left|z_{1}\right|_{H^{1}}\left|z_{2}\right|_{L^{2}}\left|z_{3}\right|_{L^{2}}$.

Thus we have proved, owing to (2.11),

$$
\left|V *\left(\overline{z_{1}} z_{2}\right) z_{3}\right|_{L^{2}} \leq\left\|V(1-\Delta)^{-1 / 2}\right\| \min _{\sigma \in \mathfrak{S}_{3}}\left|z_{\sigma(1)}\right|_{H^{1}}\left|z_{\sigma(2)}\right|_{L^{2}}\left|z_{\sigma(3)}\right|_{L^{2}}
$$

which gives

$$
\left|\left(V *\left(\overline{z_{1}} z_{2}\right)\right) z_{3}\right|_{H^{1}} \leq\left\|V(1-\Delta)^{-1 / 2}\right\| \min _{\sigma \in \mathfrak{S}_{3}}\left|z_{\sigma(1)}\right|_{H^{1}}\left|z_{\sigma(2)}\right|_{H^{1}}\left|z_{\sigma(3)}\right|_{L^{2}}
$$

Since $z \mapsto e^{i t \Delta} z$ preserves the $L^{2}$ and $H^{1}$ norms, the velocity field estimates (2.9) and (2.10) are consequences of (2.12) and (2.13).

For the sake of completeness, let us finish the proof of the global well-posedness of the Cauchy problem. The estimate (2.13) provides the Lipschitz property of $z \rightarrow V *|z|^{2} z$ in $H^{1}\left(\mathbb{R}^{d}\right)$. This implies the local in time existence and uniqueness of a solution to $(2.6)$ in $\mathcal{C}^{1}\left(\left(-T_{z_{0}}, T_{z_{0}}\right) ; H^{1}\left(\mathbb{R}^{d}\right)\right)$, and therefore the local in time existence and uniqueness of a solution to $(2.5)$ in $\mathcal{C}^{0}\left(\left(-T_{z_{0}}, T_{z_{0}}\right) ; H^{1}\left(\mathbb{R}^{d}\right)\right) \cap$ $\mathcal{C}^{1}\left(\left[-T_{z_{0}}, T_{z_{0}}\right] ; H^{-1}\left(\mathbb{R}^{d}\right)\right)$. The global in time existence then comes as usual from the control of $\left|z_{t}\right|_{H^{1}}=\left|\tilde{z}_{t}\right|_{H^{1}}$ deduced from the conservations of (2.7) and (2.8). For (2.7), take the real part of the scalar product of each member of (2.5) with $\bar{z}_{t}$. This implies $\partial_{t}\left|z_{t}\right|_{L^{2}}^{2}=0$.

For (2.8) take the scalar product with $\chi\left(-R^{-1} \Delta\right) \partial_{t} z_{t}$ where $\chi \in \mathcal{C}_{0}^{\infty}(\mathbb{R})$ satisfies $0 \leq \chi \leq 1$ and $\chi \equiv 1$ in a neighborhood of 0 , with $R>0$ :

$$
\begin{aligned}
0 & =2 \Re\left\langle\partial_{t} z_{t}, \chi\left(-R^{-1} \Delta\right) i \partial_{t} z_{t}\right\rangle \\
& =\partial_{t}\left\langle z_{t},-\Delta \chi\left(-R^{-1} \Delta\right) z_{t}\right\rangle+2 \Re\left\langle\partial_{t} z_{t}, \chi\left(-R^{-1} \Delta\right)\left[\left(V *\left|z_{t}\right|^{2}\right) z_{t}\right]\right\rangle
\end{aligned}
$$

Integrating this identity from 0 to $t$ and taking the limit as $R \rightarrow \infty$ with the help of (2.13) gives

$$
\int_{\mathbb{R}^{d}}\left|\nabla z_{t}\right|^{2} d x-\int_{\mathbb{R}^{d}}\left|\nabla z_{0}\right|^{2} d x+2 \int_{0}^{t} \Re\left\langle\partial_{s} z_{s},\left(V *\left|z_{s}\right|^{2}\right) z_{s}\right\rangle d s=0 .
$$


Due to the symmetry of $V(x)=V(-x)$, the last integrand equals

$$
\begin{aligned}
\Re\left\langle\partial_{s} z_{s},\left(V *\left|z_{s}\right|^{2}\right) z_{s}\right\rangle & =\int_{\mathbb{R}^{2 d}} \partial_{s}\left(\left|z_{s}(x)\right|^{2}\right) V(x-y)\left|z_{s}(y)\right|^{2} d x d y \\
& =\frac{1}{2} \partial_{s} \int_{\mathbb{R}^{2 d}}\left|z_{s}(x)\right|^{2} V(x-y)\left|z_{s}(y)\right|^{2} d x d y .
\end{aligned}
$$

The conserved quantities (2.7) and (2.8) combined with (2.12) imply $\left|z_{t}\right|_{H^{1}} \leq$ $C\left|z_{0}\right|_{H^{1}}$ for some constant independent of $t \in\left(-T_{z_{0}}, T_{z_{0}}\right)$, and hence $T_{z_{0}}=+\infty$.

\section{Derivation of the mean field dynamics}

This section contains the proof of our main Theorem 1.1. Below, we recall from our previous work [4] the notion of infinite-dimensional Wigner measures and collect some of their properties. We will often make use of Weyl and Wick quantization throughout this section. So, we suggest first the reading of Appendix B.

Two phase-spaces will be necessary for this analysis: $\mathcal{Z}_{0}=L^{2}\left(\mathbb{R}^{d} ; \mathbb{C}\right)$ (respectively $\mathcal{Z}_{1}=H^{1}\left(\mathbb{R}^{d} ; \mathbb{C}\right)$ ) endowed with its scalar product $\langle$,$\rangle (respectively$ $\left.\left\langle z_{1}, z_{2}\right\rangle \mathcal{Z}_{1}=\left\langle z_{1},(1-\Delta) z_{2}\right\rangle\right)$, its norm $|z|_{\mathcal{Z}_{0}}^{2}=\langle z, z\rangle=|z|_{L^{2}}^{2}$ (respectively $|z|_{\mathcal{Z}_{1}}^{2}=|z|_{H^{1}}^{2}$ ), its real scalar product $\Re\langle z, z\rangle$ (respectively $\Re\left\langle z_{1}, z_{2}\right\rangle \mathcal{Z}_{1}$ ). Only on $\mathcal{Z}_{0}$, we will use the symplectic structure with $\sigma\left(z_{1}, z_{2}\right)=\operatorname{Im}\left\langle z_{1}, z_{2}\right\rangle$. Meanwhile, the real euclidean structure on $\mathcal{Z}_{1}$ is important especially when the Liouville transport equation is written as a gradient flow according to Appendix C.

\subsection{Wigner measures}

The Wigner measures are defined after the next result proved in [4, Theorem 6.2]:

Theorem 3.1. Let $\left(\varrho_{\varepsilon}\right)_{\varepsilon \in(0, \bar{\varepsilon})}$ be a family of normal states on $\mathcal{H}$ parametrized by $\varepsilon$. Assume $\operatorname{Tr}\left[\varrho_{\varepsilon} \mathbf{N}^{\delta}\right] \leq C_{\delta}$ uniformly with respect to. $\varepsilon \in(0, \bar{\varepsilon})$ for some fixed $\delta>0$ and $C_{\delta} \in(0,+\infty)$. Then for every sequence $\left(\varepsilon_{n}\right)_{n \in \mathbb{N}}$ with $\lim _{n \rightarrow \infty} \varepsilon_{n}=0$, there exist a subsequence $\left(\varepsilon_{n_{k}}\right)_{k \in \mathbb{N}}$ and a Borel probability measure $\mu$ on $\mathcal{Z}_{0}$, such that

$$
\lim _{k \rightarrow \infty} \operatorname{Tr}\left[\varrho_{\varepsilon_{n_{k}}} b^{\mathrm{Weyl}}\right]=\int_{\mathcal{Z}_{0}} b(z) d \mu(z)
$$

for all $b$ in the cylindrical Schwartz space $\mathcal{S}_{\text {cyl }}\left(\mathcal{Z}_{0}\right)$ defined in Subsection B.1. Moreover this probability measure $\mu$ satisfies $\int_{\mathcal{Z}_{0}}|z|_{\mathcal{Z}_{0}}^{2 \delta} d \mu(z)<\infty$.

Definition 3.2. The set of Wigner measures associated with a family $\left(\varrho_{\varepsilon}\right)_{\varepsilon \in(0, \bar{\varepsilon})}$ (respectively a sequence $\left.\left(\varrho_{\varepsilon_{n}}\right)_{n \in \mathbb{N}}\right)$ which satisfies the assumptions of Theorem 3.1 is denoted by

$$
\mathcal{M}\left(\varrho_{\varepsilon}, \varepsilon \in(0, \bar{\varepsilon})\right), \quad\left(\text { respectively } \mathcal{M}\left(\varrho_{\varepsilon_{n}}, n \in \mathbb{N}\right)\right) .
$$


Moreover this definition can be extended to any family $\left(\varrho_{\varepsilon}\right)_{\varepsilon \in(0, \bar{\varepsilon})}$ such that

$$
\left\|(1+\mathbf{N})^{\delta} \varrho_{\varepsilon}(1+\mathbf{N})^{\delta}\right\|_{\mathcal{L}^{1}(\mathcal{H})} \leq C_{\delta}
$$

for some $\delta>0$ with the decomposition $\varrho_{\varepsilon}=\lambda_{\varepsilon}^{R,+} \varrho_{\varepsilon}^{R,+}-\lambda_{\varepsilon}^{R,-} \varrho_{\varepsilon}^{R,-}+i \lambda_{\varepsilon}^{I,+} \varrho_{\varepsilon}^{I,+}-$ $i \lambda_{\varepsilon}^{I,-} \varrho_{\varepsilon}^{I,-}$.

Wigner measures are in practice identified via their characteristic functions according to the relation

$$
\begin{aligned}
\mathcal{M}\left(\varrho_{\varepsilon}, \varepsilon \in(0, \bar{\varepsilon})\right)=\{\mu\} & \Leftrightarrow \lim _{\varepsilon \rightarrow 0} \operatorname{Tr}\left[\varrho_{\varepsilon} W(\sqrt{2} \pi \xi)\right]=\mathcal{F}^{-1}(\mu)(\xi) \\
& \Leftrightarrow \lim _{\varepsilon \rightarrow 0} \operatorname{Tr}\left[\varrho_{\varepsilon} W(\xi)\right]=\int_{\mathcal{Z}_{0}} e^{i \sqrt{2} \Re\langle\xi, z\rangle} d \mu(z) .
\end{aligned}
$$

The expression $\mathcal{M}\left(\varrho_{\varepsilon}, \varepsilon \in(0, \bar{\varepsilon})\right)=\{\mu\}$ simply means that the family $\left(\varrho_{\varepsilon}\right)_{\varepsilon \in(0, \bar{\varepsilon})}$ is "pure" in the sense

$$
\lim _{\varepsilon \rightarrow 0} \operatorname{Tr}\left[\varrho_{\varepsilon} b^{\text {Weyl }}\right]=\int_{\mathcal{Z}} b(z) d \mu,
$$

for all cylindrical symbol $b$ without extracting a subsequence. Actually the general case can be reduced to this one, after reducing the range of parameters to $\varepsilon \in\left\{\varepsilon_{n_{k}}, k \in \mathbb{N}\right\}$. For checking properties of the elements of $\mathcal{M}\left(\varrho_{\varepsilon}, \varepsilon \in(0, \bar{\varepsilon})\right)$, extracting a subsequence in this way allows to suppose without loss of generality $\mathcal{M}\left(\varrho_{\varepsilon}, \varepsilon \in(0, \bar{\varepsilon})\right)=\{\mu\}$.

A simple a priori estimate argument allows to extend the convergence to symbols which have a polynomial growth and to take Wick quantized symbols, with compact kernels, belonging to $\mathcal{P}_{\text {alg }}^{\infty}\left(\mathcal{Z}_{0}\right)=\oplus_{p, q \in \mathbb{N}}^{\text {alg }} \mathcal{P}_{p, q}^{\infty}\left(\mathcal{Z}_{0}\right)$ (see [4, Corollary 6.14]).

Proposition 3.3. Let $\left(\varrho_{\varepsilon}\right)_{\varepsilon \in(0, \bar{\varepsilon})}$ be a family of normal states on $\mathcal{L}(\mathcal{H})$ parametrized by $\varepsilon$ such that $\operatorname{Tr}\left[\varrho_{\varepsilon} \mathbf{N}^{\alpha}\right] \leq C_{\alpha}$ holds uniformly with respect to $\varepsilon \in(0, \bar{\varepsilon})$, for all $\alpha \in \mathbb{N}$, and such that $\mathcal{M}\left(\varrho_{\varepsilon}, \varepsilon \in(0, \bar{\varepsilon})\right)=\{\mu\}$. Then the convergence

$$
\lim _{\varepsilon \rightarrow 0} \operatorname{Tr}\left[\varrho_{\varepsilon} b^{\text {Wick }}\right]=\int_{\mathcal{Z}_{0}} b(z) d \mu(z)
$$

holds for any $b \in \mathcal{P}_{\text {alg }}^{\infty}\left(\mathcal{Z}_{0}\right)$.

A variant of the above result was provided in [4, Theorem 6.13].

Proposition 3.4. Assume that the family of operators $\left(\varrho^{\varepsilon}\right)_{\varepsilon \in(0, \bar{\varepsilon})}$ satisfies

$$
\left\|(1+\mathbf{N})^{\alpha} \varrho^{\varepsilon}(1+\mathbf{N})^{\alpha}\right\|_{\mathcal{L}^{1}(\mathcal{H})} \leq C_{\alpha}
$$

uniformly with respect to $\varepsilon \in(0, \bar{\varepsilon})$ for all $\alpha \in \mathbb{N}$. For any fixed $\beta$ belonging to $\mathcal{P}_{\text {alg }}^{\infty}\left(\mathcal{Z}_{0}\right)$ the family $\left(\beta^{\text {Wick }} \varrho^{\varepsilon}\right)_{\varepsilon \in(0, \bar{\varepsilon})}$ satisfies the assumptions of Definition 3.2 and

$$
\mathcal{M}\left(\beta^{\text {Wick }} \varrho^{\varepsilon}\right)=\left\{\beta \mu, \mu \in \mathcal{M}\left(\varrho^{\varepsilon}\right)\right\} .
$$


A closely related question is whether Wigner measures are completely identified via Wick-quantized observable. Of course this is related with the Hamburger moment problem even in finite dimension and we again refer to [4] for further discussions about this.

\subsection{Weak mean field limit of the dynamics in terms of the characteristic function}

After some extraction process and for some specific initial data $\left(\varrho_{\varepsilon}\right)_{\varepsilon \in(0, \bar{\varepsilon})}$, a family $\left(\mu_{t}\right)_{t \in \mathbb{R}}$ of measures can be defined and weakly solves a transport equation. We consider on $L_{s}^{2}\left(\mathbb{R}^{2 d}\right)$ the (unbounded) multiplication operators

$$
\tilde{V}=\frac{1}{2} V\left(x_{1}-x_{2}\right) \quad \text { and } \quad \tilde{V}_{s}=\left(e^{-i s \Delta_{x_{1}}} \otimes e^{-i s \Delta_{x_{2}}}\right) \tilde{V}\left(e^{i s \Delta_{x_{1}}} \otimes e^{i s \Delta_{x_{2}}}\right),
$$

and respectively associate to them the polynomials, well-defined on $\mathcal{Z}_{1}=H^{1}\left(\mathbb{R}^{d}\right)$,

$$
V(z)=\frac{1}{2}\left\langle z^{\otimes 2}, V(x-y) z^{\otimes 2}\right\rangle_{L_{s}^{2}\left(\mathbb{R}^{2 d}\right)} \text { and } V_{s}(z)=\left\langle z^{\otimes 2}, \tilde{V}_{s} z^{\otimes 2}\right\rangle_{L_{s}^{2}\left(\mathbb{R}^{2 d}\right)}, \quad z \in \mathcal{Z}_{1}
$$

Instead of considering

$$
\varrho_{\varepsilon}(t)=e^{-i \frac{t}{\varepsilon} H_{\varepsilon}} \varrho_{\varepsilon} e^{i \frac{t}{\varepsilon} H_{\varepsilon}},
$$

we will rather work with

$$
\tilde{\varrho}_{\varepsilon}(t)=e^{i \frac{t}{\varepsilon} H_{\varepsilon}^{0}} e^{-i \frac{t}{\varepsilon} H_{\varepsilon}} \varrho_{\varepsilon} e^{i \frac{t}{\varepsilon} H_{\varepsilon}} e^{-i \frac{t}{\varepsilon} H_{\varepsilon}^{0}}
$$

Our assumptions will be made in terms of the operator $S_{\varepsilon}(1)$ already introduced in (2.1) and which can be rewritten as a Wick observable.

Definition 3.5. The operator $S_{\varepsilon}$ is defined by

$$
S_{\varepsilon}=\sum_{n=0}^{\infty} H_{\varepsilon}^{0,(n)}+\varepsilon n+(\varepsilon n)^{3}=\mathrm{d} \Gamma(1-\Delta)+\mathbf{N}^{3},
$$

with domain $\mathcal{D}\left(S_{\varepsilon}\right)=\left\{\Psi \in \mathcal{H}: \sum_{n=0}^{\infty}\left\|\left(H_{\varepsilon}^{0,(n)}+\varepsilon n+(\varepsilon n)^{3}\right) \Psi^{(n)}\right\|_{L_{S}^{2}\left(\mathbb{R}^{d n}\right)}^{2}<\infty\right\}$ and $H_{\varepsilon}^{0,(n)}=\left.\mathrm{d} \Gamma(-\Delta)\right|_{\bigvee^{n} \mathcal{Z}_{0}}$.

Remember that it is self-adjoint with this domain (see (2.1)). Moreover it can be written $S_{\varepsilon}=s_{\varepsilon}^{\text {Wick }}$ with

$$
s_{\varepsilon}(z)=\langle z,(1-\Delta) z\rangle+\left[|z|_{\mathcal{Z}_{0}}^{6}+3 \varepsilon|z|_{\mathcal{Z}_{0}}^{4}+\varepsilon^{2}|z|_{\mathcal{Z}_{0}}^{2}\right]
$$


Proposition 3.6. Let $\left(\varrho_{\varepsilon}\right)_{\varepsilon \in(0, \bar{\varepsilon})}$ be a family of normal states on $\mathcal{H}$ satisfying for some finite constant $C>0$ the estimate

$$
\operatorname{Tr}\left[\left(1+S_{\varepsilon}\right) \varrho_{\varepsilon}\left(1+S_{\varepsilon}\right)\right] \leq C \quad \text { uniformly with respect to } \varepsilon \in(0, \bar{\varepsilon}) .
$$

The operator $S_{\varepsilon}$ is the one given in Definition 3.5 and $\tilde{\varrho}_{\varepsilon}(t)$ is the operator given by (3.3). Then for any sequence $\left(\varepsilon_{n}\right)_{n \in \mathbb{N}}$ in $(0, \bar{\varepsilon})$ such that $\lim _{n \rightarrow \infty} \varepsilon_{n}=0$ there exist a subsequence $\left(\varepsilon_{n_{k}}\right)_{k \in \mathbb{N}}$ and a family $\left(\tilde{\mu}_{t}\right)_{t \in \mathbb{R}}$ of Borel probability measures on $\mathcal{Z}_{0}$ satisfying for any $t \in \mathbb{R}$

$$
\mathcal{M}\left(\tilde{\varrho}_{\varepsilon_{n_{k}}}(t), k \in \mathbb{N}\right)=\left\{\tilde{\mu}_{t}\right\}
$$

with the Liouville equation

$$
\begin{aligned}
& \tilde{\mu}_{t}\left(e^{i \sqrt{2} \Re\langle\xi, .\rangle}\right) \\
& =\tilde{\mu}_{0}\left(e^{i \sqrt{2} \Re\langle\xi, .\rangle}\right)-2 \sqrt{2} i \int_{0}^{t} \tilde{\mu}_{s}\left(e^{i \sqrt{2} \Re\langle\xi, z\rangle} \operatorname{Im}\left\langle z^{\otimes 2}, \tilde{V}_{s} \xi \otimes z\right\rangle\right) d s, \\
& =\tilde{\mu}_{0}\left(e^{i \sqrt{2} \Re\langle\xi, .\rangle}\right)+i \int_{0}^{t} \tilde{\mu}_{s}\left(\left\{V_{s}(.) ; e^{i \sqrt{2} \Re\langle\xi, .\rangle}\right\}\right) d s, \text { for all } \xi \in \mathcal{Z}_{1} .
\end{aligned}
$$

Proof. The proof uses several preliminary lemmas stated below. By an approximation argument, we may assume $\xi \in H^{2}\left(\mathbb{R}^{d}\right)$. The first step is to prove the existence of Wigner measures defined for all times $t \in \mathbb{R}$. This is done in Proposition 3.9. Let us now prove the Liouville equation. By Lemma 3.8 we have

$\operatorname{Tr}\left[\tilde{\varrho}_{\varepsilon}(t) W(\xi)\right]=\operatorname{Tr}\left[\varrho_{\varepsilon} W(\xi)\right]+i \int_{0}^{t} \operatorname{Tr}\left[\tilde{\varrho}_{\varepsilon}(s) W(\xi) \sum_{j=1}^{4} \varepsilon^{j-1} b_{j}(s, \xi)^{\text {Wick }}\right] d s$

where $b_{j}$ are the following polynomials

$$
\begin{aligned}
& b_{1}(s, \xi)=-2 \sqrt{2} \operatorname{Im}\left\langle z^{\otimes 2}, \tilde{V}_{s} \xi \otimes z\right\rangle \\
& b_{2}(s, \xi)=-\Re\left\langle z^{\otimes 2}, \tilde{V}_{s} \xi^{\otimes 2}\right\rangle+2\left\langle\xi \vee z, \tilde{V}_{s} \xi \vee z\right\rangle \\
& b_{3}(s, \xi)=\sqrt{2} \operatorname{Im}\left\langle\xi^{\otimes 2}, \tilde{V}_{s} \xi \otimes z\right\rangle \\
& b_{4}(s, \xi)=\frac{1}{4}\left\langle\xi^{\otimes 2}, \tilde{V}_{s} \xi^{\otimes 2}\right\rangle .
\end{aligned}
$$

With the number estimate in Proposition B.1, Lemma 3.7 below will ensure that the sum in the right-hand side over $j=2, \cdots, 4$ converges to 0 when $\varepsilon \rightarrow 0$. On the other hand, the term with $j=1$ has a limit according to Lemma 3.10 applied with $\tilde{\varrho}_{\varepsilon}(t)$ after noticing that $\operatorname{Tr}\left[\left(1+S_{\varepsilon}\right) \tilde{\varrho}_{\varepsilon}(t)\left(1+S_{\varepsilon}\right)\right] \leq C^{\prime}$ owing to $\left\|\left(1+S_{\varepsilon}\right)^{ \pm 1} e^{i \frac{t}{\varepsilon} H_{0}^{\varepsilon}} e^{-i \frac{t}{\varepsilon} H_{\varepsilon}}\left(1+S_{\varepsilon}\right)^{\mp 1}\right\| \leq C^{\prime \prime}$ due to Proposition 2.3. 
The above proof is completed in essentially three steps:

1) The relation (3.5) is first established by extending Wick-calculus arguments to the case when $V$ is unbounded, and rough estimates for $b_{j}(s, \xi)^{\text {Wick }}, j=$ $1, \ldots, 4$, are given;

2) An Ascoli type argument, relying on these rough estimates allows to make the subsequence extraction $\left(\varepsilon_{n_{k}}\right)_{k \in \mathbb{N}}$ uniform for all $t \in \mathbb{R}$;

3) An additional compactness argument is given in order to ensure the convergence of the term with $j=1$ in (3.5).

\subsubsection{Wick calculus with unbounded kernels}

The results presented in this paragraph would be direct applications of the Wick calculus given in Proposition B.2 for a bounded potential $V \in L^{\infty}\left(\mathbb{R}^{d}\right)$. Although the algebra is the same as in the bounded case, justifying the formulas for unbounded potentials fulfilling (A1), (A2) and (A3) requires some analysis.

Lemma 3.7. The identity

$$
\left(V_{s}^{\text {Wick }} W(\xi)-W(\xi) V_{s}^{\text {Wick }}\right) \Psi=W(\xi)\left(\sum_{j=1}^{4} \varepsilon^{j} b_{j}(s, \xi)^{\text {Wick }}\right) \Psi
$$

holds for any $\xi \in H^{2}\left(\mathbb{R}^{d}\right)$ and $\Psi \in \mathcal{D}\left(S_{\varepsilon}\right)$, with $S_{\varepsilon}$ given by Definition 3.5. Additionally, for all $\Psi \in \mathcal{D}\left(S_{\varepsilon}\right) \subset \mathcal{D}\left(S_{\varepsilon}^{1 / 2}\right) \subset \mathcal{D}\left(\mathbf{N}^{\frac{3}{2}}\right)$, the estimates

$$
\begin{aligned}
\left\|b_{j}(s, \xi)^{\text {Wick }} \Psi\right\| & \leq C\left(1+|\xi|_{\mathcal{Z}_{1}}^{4}\right)\left\|(1+\mathbf{N})^{\frac{3}{2}} \Psi\right\| \\
& \leq C^{\prime}\left(1+|\xi|_{\mathcal{Z}_{1}}^{4}\right)\left\|\left(1+S_{\varepsilon}\right)^{1 / 2} \Psi\right\|
\end{aligned}
$$

hold uniformly with respect to $j \in\{1, \ldots, 4\}, s \in \mathbb{R}$, when $\xi \in H^{1}\left(\mathbb{R}^{d}\right)$.

Proof. We first remark that, owing to the assumption (A2) and the estimate (2.12), the polynomials $b_{j}(s, \xi), j=1, \cdots, 4$ belong to the set $\oplus_{p, q \leq 3} \mathcal{P}_{p, q}\left(\mathcal{Z}_{0}\right)$, with

$$
\left|b_{j}\right|_{\oplus_{p+q \leq 3}} \mathcal{P}_{p, q}\left(\mathcal{Z}_{0}\right) \leq C\left(1+|\xi|_{\mathcal{Z}_{1}}^{4}\right) .
$$

Hence, Proposition B.1 and Proposition 2.2 prove (3.7) with

$$
\mathcal{D}\left(S_{\varepsilon}\right) \subset \mathcal{D}\left(\mathbf{N}^{3 / 2}\right) \subset \mathcal{D}\left(b_{j}(s, \xi)^{\text {Wick }}\right), j=1, \ldots, 4, \text { and } \mathcal{D}\left(S_{\varepsilon}\right) \subset \mathcal{D}\left(V_{s}^{\text {Wick }}\right)
$$

By Proposition 2.4 the domain $\mathcal{D}\left(S_{\varepsilon}\right)$ is invariant under the action of $W(\xi)$ for all $\xi \in H^{2}\left(\mathbb{R}^{d}\right)$. A Taylor expansion yields, for all $z \in \mathcal{Z}_{1}$, the equality

$$
V_{s}\left(z+\frac{i \varepsilon}{\sqrt{2}} \xi\right)=V_{s}(z)+\sum_{j=1}^{4} \varepsilon^{j} b_{j}(s, \xi)[z] .
$$


The formula (3.6) is standard for bounded $\tilde{V}$ due to $W^{*}(\xi) b^{\text {Wick }} W(\xi)=b(.+$ $\left.\frac{i \varepsilon}{\sqrt{2}} \xi\right)^{\text {Wick }}$ when $b \in \mathcal{P}_{\text {alg }}\left(\mathcal{Z}_{0}\right)=\oplus_{p, q \in \mathbb{N}}^{\text {alg }} \mathcal{P}_{p, q}\left(\mathcal{Z}_{0}\right)$. Let us reconsider the proof of this result for our unbounded $\tilde{V}$. With the previous estimates, the quantity $\mathcal{A}(t)=$ $\left\langle\Phi, W(t \xi) V_{S}\left(.+\frac{i \varepsilon}{\sqrt{2}} t \xi\right)^{\text {Wick }} W(t \xi)^{*} \Psi\right\rangle$ is well-defined for all $\Phi, \Psi \in \mathcal{D}\left(S_{\varepsilon}\right)$ with $\mathcal{A}(t)=\sum_{j=1}^{4} \varepsilon^{j} t^{j}\left\langle\Phi, W(t \xi) b_{j}(s, \xi)^{\text {Wick }} W(t \xi)^{*} \Psi\right\rangle+\left\langle\Phi, W(t \xi) V_{s}^{\text {Wick }} W(t \xi)^{*} \Psi\right\rangle$.

We first establish equality (3.6) in a weak sense: Differentiate $\mathcal{A}(t)$ for any $\Psi, \Phi \in$ $\mathcal{D}\left(S_{\varepsilon}\right)$

$$
\begin{aligned}
& \frac{d}{d t} \mathcal{A}(t) \\
= & \sum_{j=1}^{4} \varepsilon^{j}\left\langle\Phi, W(t \xi)\left\{\left[i \phi(\xi), b_{j}(s, \xi)^{\text {Wick }}\right] t^{j}+j t^{j-1} b_{j}(s, \xi)^{\text {Wick }}\right\} W(t \xi)^{*} \Psi\right\rangle \\
& \quad+\left\langle\Phi, W(t \xi)\left[i \phi(\xi), V_{s}^{\text {Wick }}\right] W(t \xi)^{*} \Psi\right\rangle \\
= & \sum_{j=0}^{3} \varepsilon^{j} t^{j}\left\langle\Phi, W(t \xi)\left\{\left[i \phi(\xi), b_{j}(s, \xi)^{\text {Wick }}\right]+(j+1) b_{j+1}(s, \xi)^{\text {Wick }}\right\} W(t \xi)^{*} \Psi\right\rangle
\end{aligned}
$$

where $b_{0}(z)=V_{S}(z)$. Now, a direct calculation with $\phi(\xi)=\frac{1}{\sqrt{2}}\left(a(\xi)+a^{*}(\xi)\right)$ gives

$$
\left[i \phi(\xi), b_{j}(s, \xi)^{\text {Wick }}\right]=-(j+1) b_{j+1}(s, \xi)^{\text {Wick }}
$$

for $j=0, \cdots, 3$. Therefore $\mathcal{A}(1)=\mathcal{A}(0)$ and, knowing (3.8), we conclude that

$$
W(\xi)\left(V_{s}^{\text {Wick }}+\sum_{j=1}^{4} \varepsilon^{j} b_{j}(s, \xi)^{\text {Wick }}\right) W(\xi)^{*} \Psi=V_{s}^{\text {Wick }} \Psi
$$

for any $\Psi \in \mathcal{D}\left(S_{\varepsilon}\right)$. With $\Psi=W(\xi) \tilde{\Psi}$ in (3.9) for any $\tilde{\Psi} \in \mathcal{D}\left(S_{\varepsilon}\right)$, while $\Psi \in$ $\mathcal{D}\left(S_{\varepsilon}\right)$ owing to $\xi \in H^{2}\left(\mathbb{R}^{d}\right)$, the claimed equality is obtained.

Lemma 3.8. Let $\left(\varrho_{\varepsilon}\right)_{\varepsilon \in(0, \bar{\varepsilon})}$ be a family of normal states on $\mathcal{H}$. Assume that $\varrho_{\varepsilon}\left(S_{\varepsilon}+\right.$ $1) \in \mathcal{L}^{1}(\mathcal{H})$ for all $\varepsilon \in(0, \bar{\varepsilon})$, with $S_{\varepsilon}$ given by Definition 3.5 and $\tilde{\varrho}_{\varepsilon}(t)$ by (3.3). Then for any $\xi \in H^{2}\left(\mathbb{R}^{d}\right)$, the map $s \mapsto \operatorname{Tr}\left[\tilde{\varrho}_{\varepsilon}(s) W(\xi)\right]$ belongs to $C^{1}(\mathbb{R})$ and the following integral formula holds true

$$
\operatorname{Tr}\left[\tilde{\varrho}_{\varepsilon}(t) W(\xi)\right]=\operatorname{Tr}\left[\varrho_{\varepsilon} W(\xi)\right]+\frac{i}{\varepsilon} \int_{0}^{t} \operatorname{Tr}\left[\tilde{\varrho}_{\varepsilon}(s) W(\xi) \sum_{j=1}^{4} \varepsilon^{j} b_{j}(s, \xi)^{\text {Wick }}\right] d s .
$$


Proof. Write

$$
\begin{aligned}
& \operatorname{Tr}\left[\left(\tilde{\varrho}_{\varepsilon}(t)-\tilde{\varrho}_{\varepsilon}(s)\right) W(\xi)\right] \\
& =\operatorname{Tr}\left[\varrho_{\varepsilon}\left(S_{\varepsilon}+1\right)\left(S_{\varepsilon}+1\right)^{-1}\left(e^{i \frac{t}{\varepsilon} H_{\varepsilon}} e^{-i \frac{t}{\varepsilon} H_{\varepsilon}^{0}}-e^{i \frac{s}{\varepsilon} H_{\varepsilon}} e^{-i \frac{s}{\varepsilon} H_{\varepsilon}^{0}}\right) W(\xi) e^{i \frac{s}{\varepsilon} H_{\varepsilon}^{0}} e^{-i \frac{s}{\varepsilon} H_{\varepsilon}}\right] \\
& +\operatorname{Tr}\left[\varrho_{\varepsilon} e^{i \frac{t}{\varepsilon} H_{\varepsilon}} e^{-i \frac{t}{\varepsilon} H_{\varepsilon}^{0}} W(\xi)\left(S_{\varepsilon}+1\right)\left(S_{\varepsilon}+1\right)^{-1}\left(e^{i \frac{t}{\varepsilon} H_{\varepsilon}^{0}} e^{-i \frac{t}{\varepsilon} H_{\varepsilon}}-e^{i \frac{s}{\varepsilon} H_{\varepsilon}^{0}} e^{-i \frac{s}{\varepsilon} H_{\varepsilon}^{0}}\right)\right] .
\end{aligned}
$$

The following limits hold true on $\mathcal{D}\left(S_{\varepsilon}\right)$

$$
\begin{array}{r}
\lim _{s \rightarrow t} \frac{1}{t-s}\left(S_{\varepsilon}+1\right)^{-1}\left(e^{i \frac{t}{\varepsilon} H_{\varepsilon}} e^{-i \frac{t}{\varepsilon} H_{\varepsilon}^{0}}-e^{i \frac{s}{\varepsilon} H_{\varepsilon}} e^{-i \frac{s}{\varepsilon} H_{\varepsilon}^{0}}\right) \\
=\frac{i}{\varepsilon}\left(S_{\varepsilon}+1\right)^{-1} e^{i \frac{t}{\varepsilon} H_{\varepsilon}}\left(H_{\varepsilon}-H_{\varepsilon}^{0}\right) e^{-i \frac{t}{\varepsilon} H_{\varepsilon}^{0}} \\
\lim _{s \rightarrow t} \frac{1}{t-s}\left(S_{\varepsilon}+1\right)^{-1}\left(e^{i \frac{t}{\varepsilon} H_{\varepsilon}^{0}} e^{-i \frac{t}{\varepsilon} H_{\varepsilon}}-e^{i \frac{s}{\varepsilon} H_{\varepsilon}^{0}} e^{-i \frac{s}{\varepsilon} H_{\varepsilon}^{0}}\right) \\
=\frac{i}{\varepsilon}\left(S_{\varepsilon}+1\right)^{-1} e^{i \frac{t}{\varepsilon} H_{\varepsilon}^{0}}\left(H_{\varepsilon}^{0}-H_{\varepsilon}\right) e^{-i \frac{t}{\varepsilon} H_{\varepsilon}},
\end{array}
$$

by Stone's theorem and the invariance of $\mathcal{D}\left(S_{\varepsilon}\right)$ with respect to $e^{i t H_{\varepsilon}^{0}}$ and $e^{i t H_{\varepsilon}}$. Using the estimate in Proposition 2.3, the latter limits are limits in $\mathcal{L}(\mathcal{H})$ with respect to the strong convergence topology. After noticing that $\varrho_{\varepsilon} e^{i \frac{t}{\varepsilon} H_{\varepsilon}} e^{-i \frac{t}{\varepsilon} H_{\varepsilon}^{0}} W(\xi)\left(S_{\varepsilon}+1\right)$ is trace class when $\left.\xi \in H^{2}\left(\mathbb{R}^{d}\right)\right)$, owing to Proposition 2.4 and Proposition 2.3 , we take the trace and let $s \rightarrow t$. Now integrating the derivative from 0 to $t$ yields

$\operatorname{Tr}\left[\tilde{\varrho}_{\varepsilon}(t) W(\xi)\right]=\operatorname{Tr}\left[\varrho_{\varepsilon} W(\xi)\right]+\frac{i}{\varepsilon} \int_{0}^{t} \operatorname{Tr}\left[\tilde{\varrho}_{\varepsilon}(s)\left(V_{s}^{\text {Wick }} W(\xi)-W(\xi) V_{s}^{\text {Wick }}\right)\right] d s$

When $\xi \in H^{2}\left(\mathbb{R}^{d}\right)$, the equality

$$
\begin{aligned}
\operatorname{Tr} & {\left[\left(1+S_{\varepsilon}\right) \tilde{\varrho}_{\varepsilon}(s)\left(V_{s}^{\text {Wick }} W(\xi)-W(\xi) V_{s}^{\text {Wick }}\right)\left(1+S_{\varepsilon}\right)^{-1}\right] } \\
& =\operatorname{Tr}\left[\tilde{\varrho}_{\varepsilon}(s) W(\xi) \sum_{j=1}^{4} \varepsilon^{j} b_{j}(s, \xi)^{\text {Wick }}\right]
\end{aligned}
$$

makes sense, since $\left(1+S_{\varepsilon}\right) \tilde{\varrho}_{\varepsilon}(s) \in \mathcal{L}^{1}(\mathcal{H})$ and by Lemma 3.7

$$
\begin{aligned}
& \left(V_{s}^{\text {Wick }} W(\xi)-W(\xi) V_{s}^{\text {Wick }}\right)\left(1+S_{\varepsilon}\right)^{-1} \\
& \quad=W(\xi)\left[\sum_{j=1}^{4} \varepsilon^{j} b_{j}(s, \xi)^{\text {Wick }}\right]\left(1+S_{\varepsilon}\right)^{-1} \text { in } \mathcal{L}(\mathcal{H}) .
\end{aligned}
$$




\subsubsection{Subsequence extraction for all times}

The first step in the proof of Proposition 3.6 is to show the existence of Wigner measures for all times. This is accomplished below by following merely the same lines as [6, Proposition 3.3].

Proposition 3.9. Let $\left(\varrho_{\varepsilon}\right)_{\varepsilon \in(0, \bar{\varepsilon})}$ be a family of normal states on $\mathcal{H}$ satisfying for some finite constant $C>0$ the estimate

$$
\operatorname{Tr}\left[\varrho_{\varepsilon}\left(1+S_{\varepsilon}\right)\right] \leq C \quad \text { uniformly with respect to } \varepsilon \in(0, \bar{\varepsilon}) \text {. }
$$

The operator $S_{\varepsilon}$ and $\tilde{\varrho}_{\varepsilon}(t)$ are respectively given by Definition 3.5 and (3.3). Then for any sequence $\left(\varepsilon_{n}\right)_{n \in \mathbb{N}}$ in $(0, \bar{\varepsilon})$ such that $\lim _{n \rightarrow \infty} \varepsilon_{n}=0$ there exists a subsequence $\left(\varepsilon_{n_{k}}\right)_{k \in \mathbb{N}}$ and a family of Borel probability measures on $\mathcal{Z}_{0},\left(\tilde{\mu}_{t}\right)_{t \in \mathbb{R}}$, satisfying

$$
\mathcal{M}\left(\tilde{\varrho}_{\varepsilon_{n_{k}}}(t), k \in \mathbb{N}\right)=\left\{\tilde{\mu}_{t}\right\}
$$

for any $t \in \mathbb{R}$.

Proof. We only sketch the proof and essentially indicate the points which differ from [6, Proposition 3.3]. Let us write

$$
G_{\varepsilon}(t, \xi)=\operatorname{Tr}\left[\tilde{\varrho}_{\varepsilon}(t) W(\xi)\right] .
$$

Using Proposition B. 1 and $(1+\mathbf{N}) \leq 2\left(1+\mathbf{N}^{3}\right) \leq 2\left(1+S_{\varepsilon}\right)$, one can prove like in [6] that

$$
\left|G_{\varepsilon}(s, \xi)-G_{\varepsilon}(s, \eta)\right| \leq C|\xi-\eta|_{\mathcal{Z}_{0}}^{\frac{1}{2}}\left(|\xi|_{\mathcal{Z}_{0}}^{2}+|\eta|_{\mathcal{Z}_{0}}^{2}+1\right)^{\frac{1}{4}}
$$

for some constant $C>0$. We have

$$
\left|G_{\varepsilon}(t, \eta)-G_{\varepsilon}(s, \xi)\right| \leq\left|\operatorname{Tr}\left[\left(\tilde{\varrho}_{\varepsilon}(t)-\tilde{\varrho}_{\varepsilon}(s)\right) W(\xi)\right]\right|+\left|G_{\varepsilon}(s, \xi)-G_{\varepsilon}(s, \eta)\right| .
$$

On the other hand, making use of Lemma 3.8 we get

$$
\begin{aligned}
\left.\mid \operatorname{Tr}\left[\tilde{\varrho}_{\varepsilon}(t)-\tilde{\varrho}_{\varepsilon}(s)\right] W(\xi)\right] \mid \leq & \left|\int_{s}^{t} \operatorname{Tr}\left[\tilde{\varrho}_{\varepsilon}(w) \sum_{j=1}^{4} \varepsilon^{j-1} b_{j}(w, \xi)^{\text {Wick }}\right] d w\right| \\
\leq & C_{0}|t-s|\left\|\left(1+S_{\varepsilon}\right)^{1 / 2} \varrho_{\varepsilon}\left(1+S_{\varepsilon}\right)^{1 / 2}\right\|_{\mathcal{L}^{1}(\mathcal{H})} \\
& \times \sup _{w \in[t, s]} \|\left(1+S_{\varepsilon}\right)^{-1 / 2}\left[\sum_{j=1}^{4} \varepsilon^{j-1} b_{j}(w, \xi)^{\text {Wick }}\right] \\
& \times\left(1+S_{\varepsilon}\right)^{-1 / 2} \|_{\mathcal{L}(\mathcal{H})} \\
\leq & C_{1}|t-s|\left(1+|\xi| \mathcal{Z}_{1}\right)^{4},
\end{aligned}
$$


when $\xi \in H^{2}\left(\mathbb{R}^{d}\right)$. Taking an approximation $\xi_{n} \in H^{2}\left(\mathbb{R}^{d}\right), n \in \mathbb{N}$, such that $\lim _{n \rightarrow \infty}\left|\xi-\xi_{n}\right| \mathcal{Z}_{1}=0, \mathcal{Z}_{1}=H^{1}\left(\mathbb{R}^{d}\right)$, and taking the limit as $n \rightarrow \infty$ of the left-hand side with the help of (3.10), allows first to extend the previous inequality to any $\xi \in \mathcal{Z}_{1}$.

Thus, we conclude that

$$
\left|G_{\varepsilon}(t, \eta)-G_{\varepsilon}(s, \xi)\right| \leq \tilde{C}\left(|t-s|\left(|\xi|_{\mathcal{Z}_{1}}+1\right)^{4}+|\eta-\xi| \mathcal{Z}_{0} \sqrt{|\eta|_{\mathcal{Z}_{0}}^{2}+|\xi|_{\mathcal{Z}_{0}}^{2}}\right)
$$

holds for all $(s, \xi),(t, \eta) \in \mathbb{R} \times \mathcal{Z}_{1}$, uniformly with respect to. $\varepsilon \in(0, \bar{\varepsilon})$. Remember also the uniform estimate $\left|G_{\varepsilon}(s, \xi)\right| \leq 1$.

Now, we apply the same Ascoli type argument as used in [6, Proposition 3.3] in order to prove the existence of a subsequence $\left(\varepsilon_{n_{k}}\right)_{k}$ and a continuous function $G(.,):. \mathbb{R} \times \mathcal{Z}_{1} \rightarrow \mathbb{C}$ such that $G_{\varepsilon_{k}}(t, \xi)$ converges to $G(t, \xi)$ for any $t \in \mathbb{R}$ and $\xi \in \mathcal{Z}_{1}$. Furthermore (3.10) allows to extend $G(.,$.$) to a continuous function on$ $\mathbb{R} \times \mathcal{Z}_{0}$. An " $\delta / 3$ "-argument shows that for any $(t, \xi) \in \mathbb{R} \times \mathcal{Z}_{0}, \lim _{n \rightarrow \infty} G_{\varepsilon_{n}}(t, \xi)$ exists and equals $G(t, \xi)$, so that $G(t,$.$) is a norm continuous normalized function$ of positive type. Therefore, for any $t \in \mathbb{R}, G(t,$.$) is a characteristic function of$ weak distribution (or projective family of probability measures) $\tilde{\mu}_{t}$ on $\mathcal{Z}_{0}$. Finally the proof is ended as in [6, Proposition 3.3].

\subsubsection{An additional compactness argument}

Here, the compactness assumption (A3) is converted into some compactness property of the Wick symbol $b_{1}$. It allows to refer indirectly to Proposition 3.3 and to take the limit as $\varepsilon \rightarrow 0$ in the term with $j=1$ in (3.5). With the rough estimates used in Proposition 3.9, the terms in (3.5) corresponding to $j>1$ with a factor $\varepsilon^{j-1}$ will vanish as $\varepsilon \rightarrow 0$. The next lemma applied with $\tilde{\varrho}_{\varepsilon}(s)$ in the integral term of (3.5), will end the proof of Proposition 3.6.

Lemma 3.10. Let $\varrho_{\varepsilon}$ be a family of normal states on $\mathcal{H}$ satisfying for some finite constant $C>0$ the estimate

$$
\operatorname{Tr}\left[\left(1+S_{\varepsilon}\right) \varrho_{\varepsilon}\left(1+S_{\varepsilon}\right)\right] \leq C \quad \text { uniformly with respect to } \varepsilon \in(0, \bar{\varepsilon}) .
$$

Here $S_{\varepsilon}$ is given by Definition 3.5. Assume that $\mathcal{M}\left(\varrho_{\varepsilon}, \varepsilon \in(0, \bar{\varepsilon})\right)=\{\mu\}$, then for any $\xi \in \mathcal{Z}_{1}$

$$
\lim _{\varepsilon \rightarrow 0} \operatorname{Tr}\left[\varrho_{\varepsilon} W(\xi) b_{1}(s, \xi)^{\text {Wick }}\right]=\int_{\mathcal{Z}_{0}} e^{\sqrt{2} i \Re\langle\xi, z\rangle} b_{1}(s, \xi)[z] d \mu(z) .
$$

Proof. The polynomial $b_{1}(s, \xi) \in \mathcal{P}_{1,2}+\mathcal{P}_{2,1}$ splits into two similar terms, namely

$$
B_{1}(z)=\left\langle\xi \otimes z, \tilde{V}_{s} z^{\otimes 2}\right\rangle \quad \text { and } \quad B_{2}(z)=\left\langle z^{\otimes 2}, \tilde{V}_{s}(z \otimes \xi)\right\rangle
$$

with their associated operators

$$
\begin{aligned}
& \tilde{B}_{1}=(\langle\xi| \otimes \mathbb{1}) \tilde{V}_{s} \in \mathcal{L}\left(L_{s}^{2}\left(\mathbb{R}^{2 d}\right), L^{2}\left(\mathbb{R}^{d}\right)\right) \quad \text { and } \\
& \tilde{B}_{2}=\mathcal{S}_{2} \tilde{V}_{s}(\mathbb{1} \otimes|\xi\rangle) \in \mathcal{L}\left(L^{2}\left(\mathbb{R}^{d}\right), L_{s}^{2}\left(\mathbb{R}^{2 d}\right)\right) .
\end{aligned}
$$


Let $\chi \in C_{0}^{\infty}(\mathbb{R})$ with $\chi(x)=1$ if $|x| \leq 1, \chi(x)=0$ if $|x| \geq 2$ and $0 \leq \chi \leq 1$. For $m \in \mathbb{N}^{*}$, set $\chi_{m}(x)=\chi\left(\frac{x}{m}\right)$ and define

$$
\begin{aligned}
& \tilde{B}_{1, m}=\chi_{m}\left(\left|D_{x}\right|\right) \tilde{B}_{1}\left(\mathbb{1} \otimes \chi_{m}\left(\left|D_{x}\right|\right)\right) \mathcal{S}_{2} \quad \text { and } \\
& \tilde{B}_{2, m}=\mathcal{S}_{2}\left(\mathbb{1} \otimes \chi_{m}\left(\left|D_{x}\right|\right)\right) \tilde{B}_{2} \chi_{m}\left(\left|D_{x}\right|\right)
\end{aligned}
$$

as bounded operators in $\mathcal{L}\left(L_{s}^{2}\left(\mathbb{R}^{2 d}\right), L^{2}\left(\mathbb{R}^{d}\right)\right)$ and $\mathcal{L}\left(L^{2}\left(\mathbb{R}^{d}\right), L_{s}^{2}\left(\mathbb{R}^{2 d}\right)\right)$ respectively. We claim that both operators $\tilde{B}_{1, m}$ and $\tilde{B}_{2, m}$ are compact. Actually, $\tilde{B}_{2, m}=$ $\tilde{B}_{1, m}^{*}$ and

$$
\begin{aligned}
\tilde{B}_{1, m}= & \frac{1}{2}\left(\mathbb{1} \otimes e^{-i s \Delta_{x_{2}}}\right)\left(\left\langle e^{i s \Delta} \xi\right| \otimes \mathbb{1}\right)\left(e^{-i x_{1} D_{x_{2}}} \chi_{m}\left(\left|D_{x_{2}}\right|\right) V\left(x_{2}\right) \chi_{m}\left(\left|D_{x_{2}}\right|\right) e^{i x_{1} D_{x_{2}}}\right) \\
& \times\left(e^{i s \Delta_{x_{1}}} \otimes e^{i s \Delta_{x_{2}}}\right) \mathcal{S}_{2} .
\end{aligned}
$$

Moreover, the linear norm continuous application

$$
A \in \mathcal{L}\left(L^{2}\left(\mathbb{R}^{d}\right)\right) \longmapsto\left(\left\langle e^{i s \Delta} \xi\right| \otimes \mathbb{1}\right) e^{-i x_{1} D_{x_{2}}}(1 \otimes A) \in \mathcal{L}\left(L_{s}^{2}\left(\mathbb{R}^{2 d}\right), L^{2}\left(\mathbb{R}^{d}\right)\right)
$$

preserves the class of Hilbert-Schmidt operators since

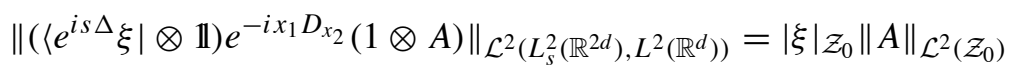

comes by computing the Schwartz kernel with $\|K\|_{\mathcal{L}^{2}\left(L^{2}(\mu) ; L^{2}(v)\right)}^{2}=\int|K(x, y)|^{2}$. $d \nu(x) d \mu(y)$. Hence it maps compact operators into compact operators, because the space of compact operators, $\mathcal{L}^{\infty}$, is the norm closure of $\mathcal{L}^{2}$ in $\mathcal{L}$. Therefore, by taking $A=\chi_{m}\left(\left|D_{x}\right|\right) V(x) \chi_{m}\left(\left|D_{x}\right|\right)$ which is compact by assumption (A3), we conclude that $\tilde{B}_{1, m}$ and $\tilde{B}_{2, m}$ are compact. Now, writing for $j=1,2$

$$
\begin{aligned}
\mid \operatorname{Tr}\left[\varrho_{\varepsilon} W(\xi) B_{j}^{\text {Wick }}\right]-\mu & \left(e^{\sqrt{2} i \Re\langle\xi, z\rangle} B_{j}(z)\right)|\leq| \operatorname{Tr}\left[\varrho_{\varepsilon} W(\xi)\left(B_{j}^{\text {Wick }}-B_{j, m}^{\text {Wick }}\right)\right] \mid(3.1 \\
+ & \operatorname{Tr}\left[\varrho_{\varepsilon} W(\xi) B_{j, m}^{\text {Wick }}\right]-\mu\left(e^{\sqrt{2} i \Re\langle\xi, z\rangle} B_{j, m}(z)\right) \mid \\
+ & \left|\mu\left(e^{\sqrt{2} i \Re\langle\xi, z\rangle} B_{j, m}(z)\right)-\mu\left(e^{\sqrt{2} i \Re\langle\xi, z\rangle} B_{j}(z)\right)\right|,
\end{aligned}
$$

with $B_{j, m} \in \mathcal{P}_{\text {alg }}^{\infty}\left(\mathcal{Z}_{0}\right)$. The right-hand side (3.13) converges to 0 owing to Proposition 3.4. Since $s-\lim _{m \rightarrow \infty} \chi_{m}\left(\left|D_{x}\right|\right)=\mathbb{1}$, the polynomials $B_{j, m}(z)$ converge to $B_{j}(z)$ for any $z \in \mathcal{Z}_{0}$, while the estimate

$$
\left|B_{j, m}(z)\right| \leq c|\xi| \mathcal{Z}_{1}\left\|(1-\Delta)^{-1 / 2} V\right\||z|_{\mathcal{Z}_{0}}^{3}
$$

holds true uniformly with respect to $m$ for some constant $c>0$. Additionally, the estimate $\operatorname{Tr}\left[\varrho_{\varepsilon} \mathbf{N}^{3 / 2}\right] \leq C$ implies

$$
\int_{\mathcal{Z}_{0}}|z|_{\mathcal{Z}_{0}}^{3} d \mu \leq C .
$$


Therefore the dominated convergence theorem applies and the right-hand side (3.14) tends to 0 as $m \rightarrow \infty$. It remains to prove the convergence of the right-hand side of (3.12). Writing

$$
\begin{aligned}
\operatorname{Tr}\left[\varrho_{\varepsilon} W(\xi)\left(B_{j}^{\text {Wick }}-B_{j, m}^{\text {Wick }}\right)\right]= & \operatorname{Tr}\left[\left(S_{\varepsilon}+1\right) \varrho_{\varepsilon}\left(S_{\varepsilon}+1\right)\left(S_{\varepsilon}+1\right)^{-1} W(\xi)\left(S_{\varepsilon}+1\right)\right. \\
& \left.\times\left(S_{\varepsilon}+1\right)^{-1}\left(B_{j}^{\text {Wick }}-B_{j, m}^{\text {Wick }}\right)\left(S_{\varepsilon}+1\right)^{-1}\right]
\end{aligned}
$$

and referring to Proposition 2.4 leads one to the estimate

$$
\left|\operatorname{Tr}\left[\varrho_{\varepsilon} W(\xi)\left(B_{j}^{\text {Wick }}-B_{j, m}^{\text {Wick }}\right)\right]\right| \leq c\left\|\left(S_{\varepsilon}+1\right)^{-1}\left(B_{j}^{\text {Wick }}-B_{j, m}^{\text {Wick }}\right)\left(S_{\varepsilon}+1\right)^{-1}\right\|_{\mathcal{L}(\mathcal{H})} .
$$

By functional calculus of strongly commuting self-adjoint operators we see that $\left(S_{\varepsilon}+1\right)^{-1}(\sqrt{\mathbf{N}}+\mathrm{d} \Gamma(1-\Delta)+1)$ is uniformly bounded with respect to $\varepsilon \in(0, \bar{\varepsilon})$. Applying Lemma B.4 (with $A=1-\Delta$ ), we conclude that

$$
\begin{aligned}
\left|\operatorname{Tr}\left[\varrho_{\varepsilon} W(\xi)\left(B_{j}^{\text {Wick }}-B_{j, m}^{\text {Wick }}\right)\right]\right| \lesssim & \left\|\left(1-\Delta_{x_{2}}\right)^{-1 / 2}\left(\tilde{B}_{j}-\tilde{B}_{j, m}\right)\left(1-\Delta_{x_{2}}\right)^{-1 / 2}\right\| \\
\lesssim & |\xi| \mathcal{Z}_{0}\left\|(1-\Delta)^{-1 / 2} V\right\|_{\mathcal{L}\left(\mathcal{Z}_{0}\right)} \\
& \times\left\|\left(1-\Delta_{x}\right)^{-1 / 2}\left(1-\chi_{m}\left(\left|D_{x}\right|\right)\right)\right\|_{\mathcal{L}\left(\mathcal{Z}_{0}\right) .}
\end{aligned}
$$

Again by functional calculus $\left\|\left(1-\Delta_{x}\right)^{-1 / 2}\left(1-\chi_{m}\left(\left|D_{x}\right|\right)\right)\right\|$ is estimated by $\frac{1}{m}$ and the right-hand side of (3.12) goes to 0 as $m \rightarrow \infty$ uniformly with respect to $\varepsilon \in(0, \bar{\varepsilon})$. Finally, a " $\delta / 3$-argument" with the established convergence of (3.12), (3.13) and (3.14) yields the result.

\subsection{Asymptotic a priori estimates}

In this section, a priori information on Wigner measures is derived from a priori estimates on the state $\varrho_{\varepsilon}$. In particular, we shall prove the next result:

Proposition 3.11. Let $S_{\varepsilon}$ be the operator given by Definition 3.5 and assume that the family of normal states $\left(\varrho_{\varepsilon}\right)_{\varepsilon \in(0, \bar{\varepsilon})}$ satisfies

$$
\forall \alpha \in \mathbb{N}, \exists C_{\alpha}>0, \forall \varepsilon \in(0, \bar{\varepsilon}), \quad \operatorname{Tr}\left[\left(1+S_{\varepsilon}\right) \varrho_{\varepsilon}\left(1+S_{\varepsilon}\right)(1+\mathbf{N})^{\alpha}\right] \leq C_{\alpha},
$$

and $\mathcal{M}\left(\varrho_{\varepsilon}, \varepsilon \in(0, \bar{\varepsilon})\right)=\{\mu\}$. Then the measure $\mu$ is carried by $\mathcal{Z}_{1}$, its restriction to $\mathcal{Z}_{1}$ is a Borel probability measure on $\mathcal{Z}_{1}$ and

$$
\int_{\mathcal{Z}_{0}}|z|_{\mathcal{Z}_{1}}^{4}|z|_{\mathcal{Z}_{0}}^{2} d \mu(z)=\int_{\mathcal{Z}_{1}}|z|_{\mathcal{Z}_{1}}^{4}|z|_{\mathcal{Z}_{0}}^{2} d \mu(z)<+\infty
$$


The proof of this proposition requires the two next results:

Lemma 3.12. Let $\tilde{b}$ be a non-negative (self-adjoint) operator on $\bigvee^{p} \mathcal{Z}_{0}$ and assume that the family of normal states $\left(\varrho_{\varepsilon}\right)_{\varepsilon \in(0, \bar{\varepsilon})}$, with $\operatorname{Tr}\left[\varrho_{\varepsilon} \mathbf{N}^{\alpha}\right] \leq C_{\alpha}$ for all $\alpha \in \mathbb{N}$, satisfies

$$
\operatorname{Tr}\left[\varrho_{\varepsilon} b^{\text {Wick }}\right] \leq C \quad \text { and } \quad \mathcal{M}\left(\varrho_{\varepsilon}, \varepsilon \in(0, \bar{\varepsilon})\right)=\{\mu\} .
$$

Then $\mathcal{Z}_{0} \ni z \mapsto b(z)=\left\langle z^{\otimes p}, \tilde{b} z^{\otimes p}\right\rangle \in[0,+\infty]$ is a Borel function on $\mathcal{Z}_{0}$ and $\int_{\mathcal{Z}_{0}} b(z) d \mu(z) \leq C$.

Proof. When $b \in \mathcal{P}_{p, p}^{\infty}\left(\mathcal{Z}_{0}\right)$ has a compact kernel $\tilde{b}$ we know after Proposition 3.3 (see $[4$, Corollary 6.14] for a complete proof) that

$$
C \geq \lim _{\varepsilon \rightarrow 0} \operatorname{Tr}\left[\varrho_{\varepsilon} b^{\text {Wick }}\right]=\int_{\mathcal{Z}_{0}} b(z) d \mu(z) .
$$

We use the fact that $\tilde{b} \rightarrow b^{\text {Wick }}$ is operator monotone, in the following sense: if the (possibly unbounded) non-negative operators $\tilde{b}_{1}, \tilde{b}_{2}$ in $\bigvee^{p} \mathcal{Z}_{0}$ satisfy $\tilde{b}_{2} \geq \tilde{b}_{1} \geq 0$, then the densely defined essentially self-adjoint operators $b_{j}^{\text {Wick }}, j=1,2$ in $\mathcal{H}$ satisfy $b_{2}^{\text {Wick }} \geq b_{1}^{\text {Wick }} \geq 0$.

By taking $\tilde{b} \in \mathcal{L}\left(\bigvee^{p} \mathcal{Z}\right)$, for $b \in \mathcal{P}_{p, p}(\mathcal{Z})$, as the supremum of $\tilde{b}_{n}$ with $\tilde{b}_{n}$ compact, we obtain firstly for all $n \in \mathbb{N}$

$$
C \geq \liminf _{\varepsilon \rightarrow 0} \operatorname{Tr}\left[\varrho_{\varepsilon}^{1 / 2} b^{\text {Wick }} \varrho_{\varepsilon}^{1 / 2}\right] \geq \lim _{\varepsilon \rightarrow 0} \operatorname{Tr}\left[\varrho_{\varepsilon}^{1 / 2} b_{n}^{\text {Wick }} \varrho_{\varepsilon}^{1 / 2}\right]=\int_{\mathcal{Z}_{0}} b_{n}(z) d \mu(z) .
$$

Secondly, the monotone convergence yields

$$
C \geq \sup _{n \in \mathbb{N}} \int_{\mathcal{Z}_{0}}\left\langle z^{\otimes p}, \tilde{b}_{n} z^{\otimes p}\right\rangle d \mu(z)=\int_{\mathcal{Z}_{0}} b(z) d \mu(z) .
$$

When $\tilde{b}$ is unbounded, it can be approximated by $\tilde{b}_{n}=\frac{\tilde{b}}{1+\frac{\tilde{b}}{\tilde{b}}} \in \mathcal{L}\left(\bigvee^{p} \mathcal{Z}_{0}\right)$, for $n \geq$ 1. Set $b_{n}(z)=\left\langle z^{\otimes p}, \tilde{b}_{n} z^{\otimes p}\right\rangle$. The function $b(z)=\left\langle z^{\otimes p}, \tilde{b} z^{\otimes p}\right\rangle=\sup _{n \in \mathbb{N}} b_{n}(z)$ is a Borel function on $\mathcal{Z}_{0}$ as a supremum of a sequence of continuous functions. The uniform estimate

$$
\operatorname{Tr}\left[\varrho_{\varepsilon} b_{n}^{\text {Wick }}\right] \leq \operatorname{Tr}\left[\varrho_{\varepsilon} b^{\text {Wick }}\right] \leq C
$$

with the result for $\tilde{b}_{n} \in \mathcal{L}\left(\bigvee^{p} \mathcal{Z}_{0}\right)$ gives $\int_{\mathcal{Z}_{0}} b_{n}(z) d \mu(z) \leq C$, for all $n \in \mathbb{N}^{*}$. Again by monotone convergence, we get

$$
\int_{\mathcal{Z}_{0}} b(z) d \mu(z)=\sup _{n \in \mathbb{N}^{*}} \int_{\mathcal{Z}_{0}} b_{n}(z) d \mu(z) \leq C .
$$


Lemma 3.13. Let $A$ be a non-negative, self-adjoint operator in $\mathcal{Z}_{0}$ with domain $\mathcal{D}(A)$. Assume that the family $\left(\varrho_{\varepsilon}\right)_{\varepsilon \in(0, \bar{\varepsilon})}$ satisfies the uniform estimate $\operatorname{Tr}\left[\varrho_{\varepsilon} \mathbf{N}^{\alpha}\right] \leq$ $C_{\alpha}$ for all $\alpha \in \mathbb{N}$, and $\mathcal{M}\left(\varrho_{\varepsilon}, \varepsilon \in(0, \bar{\varepsilon})\right)=\{\mu\}$. Then the following implications hold:

$$
\begin{aligned}
\left(\forall \varepsilon \in(0, \bar{\varepsilon}), \operatorname{Tr}\left[\varrho_{\varepsilon} \mathrm{d} \Gamma(A)\right] \leq C\right) & \Rightarrow\left(\int_{\mathcal{Z}_{0}}\langle z, A z\rangle d \mu(z) \leq C\right), \\
\left(\forall \varepsilon \in(0, \bar{\varepsilon}), \operatorname{Tr}\left[\varrho_{\varepsilon} \mathrm{d} \Gamma(A)^{2}\right] \leq C\right) & \Rightarrow\left(\int_{\mathcal{Z}_{0}}\langle z, A z\rangle^{2} d \mu(z) \leq C\right), \\
\left(\forall \varepsilon \in(0, \bar{\varepsilon}), \operatorname{Tr}\left[\varrho_{\varepsilon} \mathrm{d} \Gamma(A)^{2} \mathbf{N}\right] \leq C\right) & \Rightarrow\left(\int_{\mathcal{Z}_{0}}\langle z, A z\rangle^{2}|z|_{\mathcal{Z}_{0}}^{2} d \mu(z) \leq C\right) .
\end{aligned}
$$

In all the three cases, the measure $\mu$ is carried by the form domain $Q(A)$ of $A$.

Proof. The first implication is a direct application of Lemma 3.12 applied with

$$
b(z)=\langle z, A z\rangle \quad, \quad \tilde{b}=A \quad, \quad b^{\text {Wick }}=\mathrm{d} \Gamma(A) .
$$

The second one is a consequence of

$$
\mathrm{d} \Gamma(A)^{2}=\left(\left\langle z^{\otimes 2},(A \otimes A) z^{\otimes 2}\right\rangle\right)^{\text {Wick }}+\varepsilon \mathrm{d} \Gamma\left(A^{2}\right) \geq\left(\left\langle z^{\otimes 2},(A \otimes A) z^{\otimes 2}\right\rangle\right)^{\text {Wick }}
$$

and Lemma 3.12 with

$$
b(z)=\langle z, A z\rangle^{2} \quad \text { and } \quad \tilde{b}=A \otimes A .
$$

For the last one, notice that $\mathbf{N}=\mathrm{d} \Gamma(1)$ and $\mathrm{d} \Gamma(A)$ commute so that

$$
\mathrm{d} \Gamma(A)^{2} \mathbf{N} \geq \mathbf{N}\left(\left\langle z^{\otimes 2},(A \otimes A) z^{\otimes 2}\right\rangle\right)^{\text {Wick }} .
$$

With $\mathbf{N}=\left(|z|_{\mathcal{Z}_{0}}^{2}\right)^{\text {Wick}}$, the composition formula of Proposition B.2 (extended to an unbounded $A$ ) says that $\mathbf{N}\left(\left\langle z^{\otimes 2},(A \otimes A) z^{\otimes 2}\right\rangle\right)^{\text {Wick }}=b_{\varepsilon}^{\text {Wick }}$ with

$$
b_{\varepsilon}(z)=|z|_{\mathcal{Z}_{0}}^{2}\langle z, A z\rangle^{2}+2 \varepsilon\langle z, A z\rangle^{2} .
$$

Hence we get

$$
\mathrm{d} \Gamma(A)^{2} \mathbf{N} \geq\left(|z|_{\mathcal{Z}_{0}}^{2}\langle z, A z\rangle^{2}\right)^{\text {Wick }} .
$$

So, the result is again a consequence of Lemma 3.12 with

$$
b(z)=|z|_{\mathcal{Z}_{0}}^{2}\langle z, A z\rangle^{2} \quad, \quad \tilde{b}=\frac{1}{3}(\mathbb{1} \otimes A \otimes A+A \otimes \mathbb{1} \otimes A+A \otimes A \otimes \mathbb{1}) .
$$

For the last statement it suffices to notice that the integrand is infinite in the Borel subset of $\mathcal{Z}_{0}, \mathcal{Z}_{0} \backslash Q(A)=\left\{z \in \mathcal{Z}_{0},\langle z, A z\rangle=+\infty\right\}$. 
Proof of Proposition 3.11. With $S_{\varepsilon}=\mathrm{d} \Gamma(1-\Delta)+\mathbf{N}^{3}$, while $\mathrm{d} \Gamma(1-\Delta)$ and $\mathbf{N}$ commute, we know

$$
\left(1+S_{\varepsilon}\right)(1+\mathbf{N}) \geq \mathrm{d} \Gamma(1-\Delta) .
$$

Hence Lemma 3.13 says that the measure $\mu$ is carried by $Q(1-\Delta)=\mathcal{Z}_{1}$ with

$$
\int_{\mathcal{Z}_{0}}|z|_{\mathcal{Z}_{1}}^{2} d \mu(z)=\int_{\mathcal{Z}_{1}}|z|_{\mathcal{Z}_{1}}^{2} d \mu(z) \leq C
$$

Let us check that $\mu$ is a Borel measure on $\left(\mathcal{Z}_{1},|| \mathcal{Z}_{1}\right)$. The tightness property is given by the above inequality. According to $[4,55,59]$, it suffices to check that

$$
\begin{aligned}
& G_{1}(\xi)=\int_{\mathcal{Z}_{1}} e^{-2 i \pi \Re\langle\xi, z\rangle \mathcal{Z}_{1}} d \mu(z) \\
& \text { with }\langle u, v\rangle_{\mathcal{Z}_{1}}=\langle u,(1-\Delta) v\rangle_{\mathcal{Z}_{0}},
\end{aligned}
$$

is a positive type function which is continuous with respect to $\xi$ restricted to any finite-dimensional subspace of $\mathcal{Z}_{1}$. Consider the regularized version

$$
G_{1, n}(\xi)=\int_{\mathcal{Z}_{1}} e^{-2 i \pi \Re\left\langle\frac{A}{1+\frac{A}{n}} \xi, z\right\rangle \mathcal{Z}_{0}} d \mu(z)=\int_{\mathcal{Z}_{0}} e^{-2 i \pi \Re\left\langle\frac{A}{1+\frac{A}{n}} \xi, z\right\rangle \mathcal{Z}_{0}} d \mu(z)
$$

with $A=(1-\Delta)$. For all $\xi \in \mathcal{Z}_{1}$ the pointwise convergence

$$
\forall z \in \mathcal{Z}_{1}, \quad \lim _{n \rightarrow \infty}\left\langle\frac{A}{1+\frac{A}{n}} \xi, z\right\rangle_{\mathcal{Z}_{0}}=\langle\xi, z\rangle_{\mathcal{Z}_{1}}
$$

and the uniform bound

$$
\left|e^{-2 i \pi \Re\left\langle\frac{A}{1+\frac{A}{n}} \xi, z\right\rangle \mathcal{Z}_{0}}\right| \leq 1
$$

imply the pointwise convergence of the integrals

$$
\forall \xi \in \mathcal{Z}_{1}, \quad \lim _{n \rightarrow \infty} G_{1, n}(\xi)=G_{1}(\xi)
$$

But $G_{1, n}(\xi)$ equals $G\left(\left(1+\frac{A}{n}\right)^{-1} A \xi\right)$, where $G$ is the characteristic function of $\mu$ in $\mathcal{Z}_{0}$ :

$$
G(\eta)=\int_{\mathcal{Z}_{0}} e^{-2 i \pi \Re\langle\eta, z\rangle} \mathcal{Z}_{0} d \mu(z) .
$$

Hence for every $n \in \mathbb{N}$, the function $G_{1, n}(\xi)$ is a positive type function. As a pointwise limit of $G_{1, n}$, the function $G_{1}$ is also a positive type function.

For the continuity, the equality

$$
G_{1}(\xi)-G_{1}\left(\xi^{\prime}\right)=\int_{\mathcal{Z}_{1}}\left(e^{-i \pi \Re\left\langle\xi-\xi^{\prime}, z\right\rangle \mathcal{Z}_{1}}-e^{i \pi \Re\left\langle\xi-\xi^{\prime}, z\right\rangle \mathcal{Z}_{1}}\right) e^{-i \pi \Re\left\langle\xi+\xi^{\prime}, z\right\rangle \mathcal{Z}_{1}} d \mu(z)
$$


implies

$$
\begin{aligned}
\left|G_{1}(\xi)-G_{1}\left(\xi^{\prime}\right)\right| & \leq 2 \pi\left|\xi-\xi^{\prime}\right| \mathcal{Z}_{1} \int_{\mathcal{Z}_{1}}|z| \mathcal{Z}_{1} d \mu(z) \\
& \leq \pi\left(\int_{\mathcal{Z}_{1}} 1+|z|_{\mathcal{Z}_{1}}^{2} d \mu(z)\right)\left|\xi-\xi^{\prime}\right| \mathcal{Z}_{1},
\end{aligned}
$$

and the function $G_{1}$ is a Lipschitz function on $\mathcal{Z}_{1}$. This finishes the proof that $\mu$ is a Borel probability measure on $\mathcal{Z}_{1}$. For the inequality (3.15), it suffices to notice the inequality of (commuting) operators

$$
\left(1+S_{\varepsilon}\right)^{2}(1+\mathbf{N}) \geq\left(\mathrm{d} \Gamma(1-\Delta)^{2}\right) \mathbf{N} .
$$

Applying Lemma 3.13 yields

$$
\int_{\mathcal{Z}_{1}}|z|_{\mathcal{Z}_{1}}^{4}|z|_{\mathcal{Z}_{0}}^{2} d \mu(z) \leq C
$$

\subsection{Uniqueness of the mean field dynamics via a measure transportation technique}

Now we are in position to prove Theorem 1.1. This will be done in three steps:

1) Writing a transport equation, in a weak sense in $\mathcal{Z}_{1}$ for $\mu_{t}$;

2) Solving this equation as $\mu_{t}=\Phi(t, 0)_{*} \mu_{0}$ when the initial state $\varrho_{\varepsilon}$ fulfills strong decay estimates;

3) Relaxing the strong decay estimates.

\subsection{The transport equation on $\mathcal{Z}_{1}$}

We shall need similar notions about cylindrical functions, as those used in $\mathcal{Z}_{0}$ and recalled in Appendix B.1. Let $\mathbb{P}_{1}$ denote the set of all finite-rank orthogonal projections on $\mathcal{Z}_{1}$ and for a given $\wp \in \mathbb{P}_{1}$ let $L_{\wp, 1}(d z)$ denote the Lebesgue measure on the finite-dimensional subspace $\wp \mathcal{Z}_{1}$, with volume 1 for a $\mathcal{Z}_{1}$-orthonormal hypercube. A function $f: \mathcal{Z}_{1} \rightarrow \mathbb{C}$ is said cylindrical if there exists $\wp \in \mathbb{P}_{1}$ and a function $g$ on $\wp \mathcal{Z}_{1}$ such that $f(z)=g(\wp z)$, for all $z \in \mathcal{Z}_{1}$. In this case we say that $f$ is based on the subspace $\wp \mathcal{Z}_{1}$. The set of $\mathcal{C}_{0}^{\infty}$ (respectively $\mathcal{S}$ ) cylindrical functions on $\mathcal{Z}_{1}$, is denoted by $\mathcal{C}_{0, \text { cyl }}^{\infty}\left(\mathcal{Z}_{1}\right)$ (respectively $\mathcal{S}_{\text {cyl }}\left(\mathcal{Z}_{1}\right)$ ). We shall also need $\mathcal{C}_{0, \text { cyl }}^{\infty}\left(\mathcal{Z}_{1} \times \mathbb{R}\right)$, in which the algebraic tensor product $\mathcal{C}_{0 \text {,cyl }}^{\infty}\left(\mathcal{Z}_{1}\right) \stackrel{\text { alg }}{\otimes} \mathcal{C}_{0}^{\infty}(\mathbb{R})$ is dense. Finally the Fourier transform of elements of $\mathcal{S}_{\text {cyl }}\left(\mathcal{Z}_{1}\right)$ is given by

$$
\begin{gathered}
\mathcal{F}_{1}[f](\xi)=\int_{\wp \mathcal{Z}_{1}} f(\xi) e^{-2 \pi i \Re\langle z, \xi\rangle \mathcal{Z}_{1}} L_{\wp, 1}(d z), \\
f(z)=\int_{\wp} \mathcal{F}_{1}[f](\xi) e^{2 \pi i \Re\langle z, \xi\rangle \mathcal{Z}_{1}} L_{\wp, 1}(d \xi) .
\end{gathered}
$$


Proposition 3.14. Let $S_{\varepsilon}$ and $\tilde{\varrho}_{\varepsilon}(t)$ be the operators given by Definition 3.5 and (3.3). Assume that the family of normal states $\left(\varrho_{\varepsilon}\right)_{\varepsilon \in(0, \bar{\varepsilon})}$ satisfies

$$
\forall \alpha \in \mathbb{N}, \exists C_{\alpha}>0, \forall \varepsilon \in(0, \bar{\varepsilon}), \quad \operatorname{Tr}\left[\left(1+S_{\varepsilon}\right) \varrho_{\varepsilon}\left(1+S_{\varepsilon}\right)(1+\mathbf{N})^{\alpha}\right] \leq C_{\alpha},
$$

and consider a subsequence $\left(\varepsilon_{k}\right)_{k \in \mathbb{N}}, \varepsilon_{k} \stackrel{k \rightarrow \infty}{\rightarrow} 0$ such that

$$
\mathcal{M}\left(\tilde{\varrho}_{\varepsilon_{k}}(t), k \in \mathbb{N}\right)=\left\{\tilde{\mu}_{t}\right\}
$$

according to Proposition 3.6. Then the measure $\tilde{\mu}_{t}$ is a Borel probability measure on $\mathcal{Z}_{1}$ which satisfies the following properties:

- $\int_{\mathcal{Z}_{1}}|z|_{\mathcal{Z}_{1}}^{2} d \tilde{\mu}_{t}(z)+\int_{\mathcal{Z}_{1}}|z|_{\mathcal{Z}_{1}}^{4}|z|_{\mathcal{Z}_{0}}^{2} d \tilde{\mu}_{t}(z) \leq C^{\prime}$ for some $C^{\prime}$ independent of $t \in$ $\mathbb{R}$;

- When $\left(e_{n}\right)_{n \in \mathbb{N}^{*}}$ is a Hilbert basis of $\mathcal{Z}_{1}$ and $\mathcal{Z}_{1}$ is endowed with the distance $d_{\omega}\left(z_{1}, z_{2}\right)=\sqrt{\sum_{n \in \mathbb{N}^{*}} \frac{\left|\left\langle z_{1}-z_{2}, e_{n}\right\rangle\right|^{2}}{(1+n)^{2}}}, \tilde{\mu}_{t}$ is narrowly continuous with respect to $t \in \mathbb{R}$;

- The measure $\mu_{t}$ is a solution to the Liouville equation

$$
\partial_{t} \tilde{\mu}_{t}+i\left\{V_{t}, \tilde{\mu}_{t}\right\}=0
$$

in a weak sense, namely

$$
\forall f \in \mathcal{C}_{\mathrm{cyl}, 0}^{\infty}\left(\mathcal{Z}_{1} \times \mathbb{R}\right), \quad \int_{\mathbb{R}} \int_{\mathcal{Z}_{1}}\left(\partial_{t} f+i\left\{V_{t}, f\right\}\right) d \tilde{\mu}_{t}(x) d t=0 .
$$

Proof. Proposition 2.3 and the commutations $\left[e^{i t H_{\varepsilon}}, \mathbf{N}\right]=\left[e^{i t H_{\varepsilon}^{0}}, \mathbf{N}\right]=0$ ensure $\forall \alpha \in \mathbb{N}, \exists C_{\alpha}^{\prime}>0, \forall t \in \mathbb{R}, \forall \varepsilon \in(0, \bar{\varepsilon}), \operatorname{Tr}\left[\left(1+S_{\varepsilon}\right) \tilde{\varrho}_{\varepsilon}(t)\left(1+S_{\varepsilon}\right)(1+\mathbf{N})^{\alpha}\right] \leq C_{\alpha}^{\prime}$. Proposition 3.11 and (3.16) applied for any $t \in \mathbb{R}$, provides the first results. It remains to check the narrow continuity and the Liouville equation.

a) Take the $\mathcal{Z}_{1}$-characteristic function

$$
G_{1}(\eta, t)=\tilde{\mu}_{t}\left(e^{-2 i \pi \Re\langle\eta, z\rangle \mathcal{z}_{1}}\right) .
$$

Inequality (3.17) and the uniform estimate $\int_{\mathcal{Z}_{1}}\left(1+|z|_{\mathcal{Z}_{1}}^{2}\right) d \tilde{\mu}_{t}(z) \leq 1+C^{\prime}$ ensure that the inequality

$$
\left|G_{1}(\eta, t)-G_{1}\left(\eta^{\prime}, t\right)\right| \leq \pi\left(1+C^{\prime}\right)\left|\eta-\eta^{\prime}\right| \mathcal{Z}_{1}
$$

holds uniformly for all $\eta, \eta^{\prime} \in \mathcal{Z}_{1}$ and all $t \in \mathbb{R}$. From the identity (3.4), we deduce $\tilde{\mu}_{t^{\prime}}\left(e^{i \sqrt{2} \Re\langle\xi, .\rangle}\right)-\tilde{\mu}_{t}\left(e^{i \sqrt{2} \Re\langle\xi, .\rangle}\right)=-2 \sqrt{2} i \int_{t}^{t^{\prime}} \tilde{\mu}_{s}\left(e^{i \sqrt{2} \Re\langle\xi, z\rangle} \operatorname{Im}\left\langle z^{\otimes 2}, \tilde{V}_{s} \xi \otimes z\right\rangle\right) d s$. 
Estimate (2.13) implies

$$
\left|\operatorname{Im}\left\langle z^{\otimes 2}, \tilde{V}_{s}(\xi \otimes z)\right\rangle\right| \leq C|z|_{\mathcal{Z}_{1}}^{2}|z| \mathcal{Z}_{0}|\xi|_{H^{-1}\left(\mathbb{R}^{d}\right)}
$$

Taking $\xi=\sqrt{2} \pi(1-\Delta) \eta$ with $\eta \in H^{1}(\mathbb{R})=\mathcal{Z}_{1}$ leads to

$$
\left|G_{1}(\eta, t)-G_{1}\left(\eta, t^{\prime}\right)\right| \leq 4 \pi C|\eta| \mathcal{Z}_{1}\left|t-t^{\prime}\right| \sup _{s \in\left[t, t^{\prime}\right]} \int_{\mathcal{Z}_{1}}|z|_{\mathcal{Z}_{1}}^{2}|z| \mathcal{Z}_{0} d \tilde{\mu}_{s}(z)
$$

and, with the uniform estimate $\int_{\mathcal{Z}_{1}}|z|_{\mathcal{Z}_{1}}^{4}|z|_{\mathcal{Z}_{0}}^{2} d \tilde{\mu}_{t}(z) \leq C^{\prime}$, to

$$
\forall \eta \in \mathcal{Z}_{1}, \forall t, t^{\prime} \in \mathbb{R}, \quad\left|G_{1}(\eta, t)-G_{1}\left(\eta, t^{\prime}\right)\right| \leq 4 \pi C\left(1+C^{\prime}\right)|\eta|_{\mathcal{Z}_{1}}\left|t-t^{\prime}\right| .
$$

When $g \in \mathcal{S}_{\text {cyl }}\left(\mathcal{Z}_{1}\right)$, based on $\wp \mathcal{Z}_{1}$, the relation

$$
\int_{\mathcal{Z}_{1}} g(z) d \tilde{\mu}_{t}(z)=\int_{\wp \mathcal{Z}_{1}} \mathcal{F}_{1}[g](\eta) G_{1}(\eta, t) d L_{\wp, 1}(z),
$$

combined with the continuity properties (3.19) and (3.20), implies that $t \rightarrow$ $\int_{\mathcal{Z}_{1}} g(z) d \tilde{\mu}_{t}(z)$ is continuous. This continuity holds for all $g \in \mathcal{S}_{\text {cyl }}\left(\mathcal{Z}_{1}\right)$. The uniform weak tightness property $\int_{\mathcal{Z}_{1}}|z|_{\mathcal{Z}_{1}}^{2} d \tilde{\mu}_{t}(z) \leq C^{\prime}$ and Lemma 5.12-f) in [3] ensure that $t \mapsto \tilde{\mu}_{t}$ is narrowly continous when $\mathcal{Z}_{1}$ is endowed with the distance $d_{\omega}$.

b) Integrating (3.4) with $\mathcal{F}_{1}[g](\eta) L_{\wp, 1}(d z)$ also provides

$$
\int_{\mathcal{Z}_{1}} g(z) d \tilde{\mu}_{t}(z)=\int_{\mathcal{Z}_{1}} g(z) d \tilde{\mu}_{0}(z)+i \int_{0}^{t} \int_{\mathcal{Z}_{1}}\left\{V_{s}, g\right\}(z) d \tilde{\mu}_{s}(z) d s .
$$

Hence for any $g \in \mathcal{S}_{\text {cyl }}\left(\mathcal{Z}_{1}\right)$, the function $I_{g}: t \mapsto \int_{\mathcal{Z}_{1}} g(z) d \tilde{\mu}_{t}(z)$ belongs to $\mathcal{C}^{1}(\mathbb{R})$ with

$$
\partial_{t} I_{g}(t)=i \int_{\mathcal{Z}_{1}}\left\{V_{t}, g\right\}(z) d \tilde{\mu}_{t}(z) .
$$

By multiplying the above relation by $\varphi(t)$, with $\varphi \in \mathcal{C}_{0}^{\infty}(\mathbb{R})$, and integrating by part proves (3.18) when $f(t, z)=\varphi(t) g(z)$. We conclude by the density of $\mathcal{C}_{0, \text { cyl }}^{\infty}\left(\mathcal{Z}_{1}\right) \stackrel{\text { alg }}{\otimes} \mathcal{C}_{0}^{\infty}(\mathbb{R})$ in $\mathcal{C}_{0, \text { cyl }}^{\infty}\left(\mathcal{Z}_{1} \times \mathbb{R}\right)$

\subsection{Uniqueness of the measure for regular initial data}

According to the notation of [3] and Appendix $\mathrm{C}$, we consider the space $\operatorname{Prob}_{2}\left(\mathcal{Z}_{1}\right)$ of Borel probability measures $\mu$ such that

$$
\int_{\mathcal{Z}_{1}}|z|_{\mathcal{Z}_{1}}^{2} d \mu(z)<+\infty
$$


On this space, we introduce the Wasserstein distance

$$
W_{2}\left(\mu_{1}, \mu_{2}\right)=\left[\min _{\mu \in \Gamma\left(\mu_{1}, \mu_{2}\right)} \int_{\mathcal{Z}_{1}^{2}}\left|z_{1}-z_{2}\right|_{\mathcal{Z}_{1}}^{2} d \mu\left(z_{1}, z_{2}\right)\right]^{1 / 2}
$$

where $\Gamma\left(\mu_{1}, \mu_{2}\right)$ is the set of Borel probability measures $\mu$ on $\mathcal{Z}_{1} \times \mathcal{Z}_{1}$ with the marginals $\left(\Pi_{1}\right)_{*} \mu=\mu_{1}$ and $\left(\Pi_{2}\right)_{*} \mu=\mu_{2}$.

Proposition 3.15. Let $S_{\varepsilon}$ and $\tilde{\varrho}_{\varepsilon}(t)$ be the operators given by Definition 3.5 and (3.3). Assume that the family of normal states $\left(\varrho_{\varepsilon}\right)_{\varepsilon \in(0, \bar{\varepsilon})}$ satisfies

$$
\forall \alpha \in \mathbb{N}, \exists C_{\alpha}>0, \forall \varepsilon \in(0, \bar{\varepsilon}), \quad \operatorname{Tr}\left[\left(1+S_{\varepsilon}\right) \varrho_{\varepsilon}\left(1+S_{\varepsilon}\right)(1+\mathbf{N})^{\alpha}\right] \leq C_{\alpha}
$$

and $\mathcal{M}\left(\varrho_{\varepsilon}, \varepsilon \in(0, \bar{\varepsilon})\right)=\left\{\mu_{0}\right\}$.

Then for any time $t \in \mathbb{R}$, the family $\left(\varrho_{\varepsilon}(t)=e^{-i \frac{t}{\varepsilon} H_{\varepsilon}} \varrho_{\varepsilon} e^{i \frac{t}{\varepsilon} H_{\varepsilon}}\right)_{\varepsilon \in(0, \bar{\varepsilon})}$ admits a unique Wigner measure $\mu_{t}=\Phi(t, 0)_{*} \mu_{0}$, where $\Phi$ is the Hartree flow defined by (1.3) on $\mathcal{Z}_{1}$. It is a Borel probability measure on $\mathcal{Z}_{1}$ with $t \mapsto \mu_{t}$ being an absolutely continuous curve in $\operatorname{Prob}_{2}\left(\mathcal{Z}_{1}\right)$ with respect to the Wasserstein distance $W_{2}$ and which satisfies

$$
\forall t \in \mathbb{R}, \quad \int_{\mathcal{Z}_{1}}|z|_{\mathcal{Z}_{1}}^{4}|z|_{\mathcal{Z}_{0}}^{2} d \mu_{t}(z) \leq C .
$$

Proof. We still start with the state $\tilde{\varrho}_{\varepsilon}(t)$ defined in (3.3). Proposition 2.6 says that the group $\Phi(t, s)$ associated with $(1.3)$ and the dynamical system $\tilde{\Phi}(t, s)$ associated with

$$
i \partial_{t} z=v(t, z) \quad, \quad v(t, z)=e^{-i t \Delta}\left(\left[V *\left|e^{i t \Delta} z\right|^{2}\right] e^{i t \Delta} z\right)
$$

are well-defined on $\mathcal{Z}_{1}$. Further it gives the estimate for the velocity field

$$
|v(t, z)| \mathcal{Z}_{1} \leq\left\|V(1-\Delta)^{-1 / 2}\right\||z|_{\mathcal{Z}_{1}}^{2}|z| \mathcal{Z}_{0} .
$$

When $\tilde{\mu}_{t}$ is the Wigner measure defined for all times and associated with a subsequence $\left(\varepsilon_{n_{k}}\right)_{k \in \mathbb{N}}$, we obtain

$$
\forall t \in \mathbb{R}, \quad \int_{\mathcal{Z}_{1}}|v(t, z)|_{\mathcal{Z}_{1}}^{2} d \tilde{\mu}_{t}(z) \leq C \int_{\mathcal{Z}_{1}}|z|_{\mathcal{Z}_{1}}^{4}|z|_{\mathcal{Z}_{0}}^{2} d \tilde{\mu}_{t}(z) \leq C^{\prime} .
$$

With Proposition 3.14, $t \mapsto \tilde{\mu}_{t}$ is narrowly continuous (on $\left(\mathcal{Z}_{1}, d_{\omega}\right)$ ) with respect to time $t \in \mathbb{R}$. According to Lemma C.7, the Liouville equation (3.18) is nothing but the weak form of

$$
\partial_{t} \mu+\nabla^{T}(v(t, z) \mu)=0 .
$$

According to Proposition C.1, the curve $\mathbb{R} \ni t \mapsto \tilde{\mu}_{t} \in \operatorname{Prob}_{2}\left(\mathcal{Z}_{1}\right)$ is absolutely continuous for the Wasserstein distance $W_{2}$.

Therefore all conditions of Proposition C.8 are fulfilled and hence we deduce that $\tilde{\mu}_{t}=\tilde{\Phi}(t, 0)_{*} \mu_{0}$. Moreover this uniqueness implies $\mathcal{M}\left(\tilde{\varrho}_{\varepsilon}(t), \varepsilon \in(0, \bar{\varepsilon})\right)=$ $\left\{\tilde{\mu}_{t}\right\}$ for the whole family $\left(\tilde{\varrho}_{\varepsilon}(t)\right)_{\varepsilon \in(0, \bar{\varepsilon})}$ and all times $t \in \mathbb{R}$.

Going back to $\varrho_{\varepsilon}(t)=e^{-i \frac{t}{\varepsilon} H_{\varepsilon}^{0}} \tilde{\varrho}_{\varepsilon}(t) e^{i \frac{t}{\varepsilon} H_{\varepsilon}^{0}}$, it gives $\mu_{t}=\Phi(t, 0)_{*} \mu_{0}$.

The last uniform estimate is given by Proposition 3.14. 


\subsection{Evolution of the Wigner measure for general data}

We follow the truncation scheme used in [6]. When the initial data satisfies only

$$
\left\|\left(\mathbf{N}+H_{\varepsilon}^{0}\right)^{\delta / 2} \varrho_{\varepsilon}\left(\mathbf{N}+H_{\varepsilon}^{0}\right)^{\delta / 2}\right\| \leq C_{\delta}
$$

for some $\delta>0$, we approximate $\varrho_{\varepsilon}$ by

$$
\varrho_{\varepsilon, R}=\frac{1}{\operatorname{Tr}\left[\chi_{R}\left(\mathbf{N}, H_{\varepsilon}^{0}\right) \varrho_{\varepsilon} \chi_{R}\left(\mathbf{N}, H_{\varepsilon}^{0}\right)\right]} \chi_{R}\left(\mathbf{N}, H_{\varepsilon}^{0}\right) \varrho_{\varepsilon} \chi_{R}\left(\mathbf{N}, H_{\varepsilon}^{0}\right)
$$

as $R \rightarrow+\infty$ where $\chi_{R}(n, h)=\chi\left(\frac{n}{R}, \frac{h}{R}\right)$, with $0 \leq \chi \leq 1, \chi \in \mathcal{C}_{0}^{\infty}\left(\mathbb{R}^{2}\right)$ and $\chi \equiv 1$ in a neighborhood of 0 . The time evolved state is defined by

$$
\varrho_{\varepsilon, R}(t)=e^{-i \frac{t}{\varepsilon} H_{\varepsilon}} \varrho_{\varepsilon, R} e^{i \frac{t}{\varepsilon} H_{\varepsilon}} .
$$

The assumptions ensure that for all times

$$
\left\|\varrho_{\varepsilon}(t)-\varrho_{\varepsilon, R}(t)\right\|_{\mathcal{L}^{1}(\mathcal{H})} \leq v(R)
$$

with $v$ independent of $(t, \varepsilon)$ and $\lim _{R \rightarrow \infty} v(R)=0$. We recall the Proposition 2.10 of [6].

Proposition 3.16. Let $\left(\varrho_{\varepsilon}^{j}\right)_{\varepsilon \in(0, \bar{\varepsilon})}, j=1,2$, be two families (or sequences) of normal states on $\mathcal{H}$ such that $\operatorname{Tr}\left[\varrho_{\varepsilon}^{j} \mathbf{N}^{\delta}\right] \leq C_{\delta}$ uniformly with respect to $\varepsilon \in(0, \bar{\varepsilon})$ for some $\delta>0$ and $C_{\delta} \in(0,+\infty)$. Assume further $\mathcal{M}\left(\varrho_{\varepsilon}^{j}, \varepsilon \in(0, \bar{\varepsilon})\right)=\left\{\mu_{j}\right\}$ for $j=1,2$. Then

$$
\int\left|\mu_{1}-\mu_{2}\right| \leq \liminf _{\varepsilon \rightarrow 0}\left\|\varrho_{\varepsilon}^{1}-\varrho_{\varepsilon}^{2}\right\|_{\mathcal{L}^{1}(\mathcal{H})} .
$$

End of the proof of Theorem 1.1. For $R \in(0,+\infty)$, the state $\varrho_{\varepsilon, R}$ fulfills the conditions of Proposition 3.15 except the uniqueness of the Wigner measure at time $t=0$. Out of any sequence $\left(\varepsilon_{n}\right)_{n \in \mathbb{N}}$, a subsequence $\left(\varepsilon_{n_{k}}\right)_{k \in \mathbb{N}}$ can be extracted in order to ensure

$$
\mathcal{M}\left(\varrho_{\varepsilon_{n_{k}}, R}, k \in \mathbb{N}\right)=\left\{\mu_{0, R}\right\} .
$$

Thus after this extraction we obtain

$$
\forall t \in \mathbb{R}, \quad \mathcal{M}\left(\varrho_{\varepsilon_{n_{k}}, R}(t), k \in \mathbb{N}\right)=\left\{\Phi(t, 0)_{*} \mu_{0, R}\right\} .
$$

Take $t \in \mathbb{R}$ and let $\mu$ belong to $\mathcal{M}\left(\varrho_{\varepsilon}(t), \varepsilon \in(0, \bar{\varepsilon})\right)$. There exists a sequence $\left(\varepsilon_{n}\right)_{n \in \mathbb{N}}$ such that

$$
\mathcal{M}\left(\varrho_{\varepsilon_{n}}(t), n \in \mathbb{N}\right)=\{\mu\} .
$$


After extracting a subsequence like above and by using Proposition 3.16, we obtain

$$
\int\left|\mu-\Phi(t, 0)_{*} \mu_{0}\right| \leq \int\left|\mu-\Phi(t, 0)_{*} \mu_{0, R}\right|+\int\left|\mu_{0, R}-\mu_{0}\right| \leq 2 v(R),
$$

since the total variation of $\Phi(t, 0)_{*} \mu_{0, R}-\Phi(t, 0)_{*} \mu_{0}$ and $\mu_{0, R}-\mu_{0}$ are equal. Taking the limit as $R \rightarrow \infty$ implies $\mu=\Phi(t, 0)_{*} \mu_{0}$ and therefore

$$
\mathcal{M}\left(\varrho_{\varepsilon}, \varepsilon \in(0, \bar{\varepsilon})\right)=\left\{\Phi(t, 0)_{*} \mu_{0}\right\} .
$$

This also proves that $\lim _{R \rightarrow \infty} \int_{\mathcal{Z}_{0}}\left|\mu_{t}-\Phi(t, 0)_{*} \mu_{0, R}\right|=0$, while all the measures $\Phi(t, 0)_{*} \mu_{0, R}$ are Borel probability measures carried by, and on, $\mathcal{Z}_{1}$. This implies that $\mu_{t}$ is carried by $\mathcal{Z}_{1}$ and is also a Borel measure on $\mathcal{Z}_{1}$. This ends the proof of Theorem 1.1.

\section{Complements}

Additional results are given in the three first paragraphs, concerned with the BBGKY hierarchy or the propagation of energy. The fourth one shows some examples and the last one is an informal discussion about the classical mean field problem.

\subsection{BBGKY hierarchy}

Although the analysis here is different from our previous work [6] it is possible to combine them, in order to strengthen the result of Theorem 1.1. It is also interesting to reformulate our result in terms of reduced density matrices since, in the literature, several mathematical results on mean field limit use the BBGKY hierarchy method (see for example $[8,9,42]$ ). For a family of normal states $\left(\varrho_{\varepsilon}\right)_{\varepsilon \in(0, \bar{\varepsilon})}$ on $\mathcal{H}$ and $p \in \mathbb{N}$, the reduced density matrices $\gamma_{\varepsilon}^{(p)} \in \mathcal{L}^{1}\left(L_{S}^{2}\left(\mathbb{R}^{d p}\right)\right)$ is defined according to

$$
\operatorname{Tr}\left[\gamma_{\varepsilon}^{(p)} \tilde{b}\right]=\frac{\operatorname{Tr}\left[\varrho_{\varepsilon}\right]}{\operatorname{Tr}\left[\varrho_{\varepsilon}\left(|z|^{2 p}\right)^{\text {Wick }}\right]} \operatorname{Tr}\left[\varrho_{\varepsilon} b^{\text {Wick}}\right], \quad \forall \tilde{b} \in \mathcal{L}\left(L_{s}^{2}\left(\mathbb{R}^{d p}\right)\right),
$$

with the convention that the right-hand side is 0 when $\operatorname{Tr}\left[\varrho_{\varepsilon}\left(|z|^{2 p}\right)^{\text {Wick }}\right]=0$ and $p>0$.

Theorem 4.1. Let $\left(\varrho_{\varepsilon}\right)_{\varepsilon \in(0, \bar{\varepsilon})}$ be a family of normal states on $\mathcal{H}$, satisfying the hypothesis of Theorem 1.1, with a single Wigner measure $\mu_{0}$ such that

$$
\forall \alpha \in \mathbb{N}, \quad \lim _{\varepsilon \rightarrow 0} \operatorname{Tr}\left[\varrho_{\varepsilon} \mathbf{N}^{\alpha}\right]=\int_{\mathcal{Z}_{0}}|z|^{2 \alpha} d \mu_{0}(z)<+\infty .
$$

Then for all $t \in \mathbb{R}$, the convergence

$$
\lim _{\varepsilon \rightarrow 0} \operatorname{Tr}\left[\varrho_{\varepsilon}(t) b^{\text {Wick }}\right]=\int_{\mathcal{Z}_{0}} b(\Phi(t, 0) z) d \mu_{0}(z)=\int_{\mathcal{Z}_{0}} b(z) d \mu_{t}(z)
$$


holds for any $b \in \mathcal{P}_{\mathrm{alg}}\left(\mathcal{Z}_{0}\right)=\oplus_{p, q \in \mathbb{N}}^{\mathrm{alg}} \mathcal{P}_{p, q}\left(\mathcal{Z}_{0}\right)$, with $\mu_{t}=\Phi(t, 0)_{*} \mu_{0}$. Finally, the convergence of the reduced density matrices

$$
\lim _{\varepsilon \rightarrow 0} \gamma_{\varepsilon}^{(p)}(t)=\frac{1}{\int_{\mathcal{Z}_{0}}|z|^{2 p} d \mu_{t}(z)} \int_{\mathcal{Z}_{0}}\left|z^{\otimes p}\right\rangle\left\langle z^{\otimes p}\right| d \mu_{t}(z)
$$

holds in the $\mathcal{L}^{1}\left(L_{s}^{2}\left(\mathbb{R}^{d p}\right)\right)$-norm for all $p \in \mathbb{N}$.

Proof. By Theorem 1.1 the the family of normal states $\left(\varrho_{\varepsilon}(t)\right)_{\varepsilon \in(0, \bar{\varepsilon})}$ admits a single Wigner measure $\mu_{t}$ equal to $\Phi(t, 0)_{*} \mu_{0}$. Since the quantum and classical flows preserve the total number, the state $\varrho_{\varepsilon}(t)$ satisfies as well condition (4.2) for any time $t \in \mathbb{R}$. Then [6, Proposition 2.11,2.13] provide the claimed results.

\subsection{Moment upper bounds}

In [4], it was proved that the sole a priori estimate $\operatorname{Tr}\left[\varrho_{\varepsilon} \mathbf{N}^{\delta}\right] \leq C_{\delta}$ for a given $\delta>0$ (possibly small), with $\mathcal{M}\left(\varrho_{\varepsilon}, \varepsilon \in(0, \bar{\varepsilon})\right)=\{\mu\}$ leads to

$$
\int_{\mathcal{Z}_{0}}|z|^{2 \delta} d \mu(z)<+\infty .
$$

The a priori estimate, assumed in Theorem 1.1 at time $t=0$, leads to

$$
\int_{\mathcal{Z}_{1}}|z|_{\mathcal{Z}_{1}}^{2 \delta} d \mu(z)<+\infty
$$

according to the following result which is a variation of Lemma 3.12:

Proposition 4.2. Let $(A, \mathcal{D}(A))$ be a self-adjoint operator on $\mathcal{Z}_{0}$ such that $A \geq 1$.

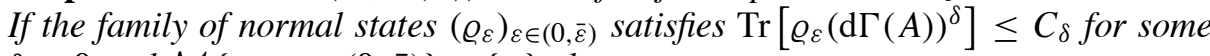
$\delta>0$ and $\mathcal{M}\left\{\varrho_{\varepsilon}, \varepsilon \in(0, \bar{\varepsilon})\right\}=\{\mu\}$, then

$$
\int_{\mathcal{Z}_{0}}\langle z, A z\rangle^{\delta} d \mu(z)<+\infty
$$

Proof. By Wick calculus (see Proposition B.2 when A is bounded), one gets

$$
\mathrm{d} \Gamma(A)^{k} \geq\left(\langle z, A z\rangle^{k}\right)^{\text {Wick }}, \forall k \in \mathbb{N} .
$$

Let $\left(e_{j}\right)_{j \in \mathbb{N}}$ be an orthonormal basis of $\mathcal{Z}_{0}$ such that $e_{j} \in \mathcal{D}(A)$ for all $j \in \mathbb{N}$, and set

$$
A_{J}=\sum_{j=0}^{J} A^{1 / 2}\left|e_{j}\right\rangle\left\langle e_{j}\left|A^{1 / 2}=\sum_{j=0}^{J}\right| A^{1 / 2} e_{j}\right\rangle\left\langle A^{1 / 2} e_{j}\right| .
$$


The inequality $A^{\otimes k} \geq A_{J}^{\otimes k}$ holds for all $J \in \mathbb{N}$, while $\tilde{b} \rightarrow b^{\text {Wick }}$ is operator monotone when restricted to operators $\tilde{b}$ acting in $\bigvee^{2 k} \mathcal{Z}_{0}$. Therefore, we obtain

$$
\begin{aligned}
(1+\mathrm{d} \Gamma(A))^{n} & =\sum_{k=0}^{n} C_{n}^{k} \mathrm{~d} \Gamma(A)^{k} \geq\left(\sum_{k=0}^{n} C_{n}^{k}\langle z, A z\rangle^{k}\right)^{\text {Wick }} \\
& \geq\left(\sum_{k=0}^{n} C_{n}^{k}\left\langle z, A_{J} z\right\rangle^{k}\right)^{\text {Wick }}=\left[\left(1+\left\langle z, A_{J} z\right\rangle\right)^{n}\right]^{\text {Wick }} .
\end{aligned}
$$

We shall use the same argument as the one in [4, Theorem 6.2] when $A=\mathrm{Id}$, relying on the semiclassical calculus in finite dimension (see $[12,40,50,53,57])$.

Let $\wp_{J}$ be the orthogonal projection from $\mathcal{Z}_{0}$ onto $\oplus_{j=0}^{J} A^{1 / 2} e_{j}$. The symbol $1+\left\langle z, A_{J} z\right\rangle$ is a cylindrical symbol based on $\wp_{J} \mathcal{Z}_{0}$. Since $\operatorname{ker} A^{1 / 2}=\{0\}$ and $e_{0}, \ldots, e_{J}$ are linearly independent, the symbol

$$
\left(1+\left\langle z, A_{J} z\right\rangle\right)=1+\sum_{j=0}^{J}\left|\left\langle A^{1 / 2} e_{j}, z\right\rangle\right|^{2}
$$

is an elliptic symbol on $\wp_{J} \mathcal{Z}_{0} \sim \mathbb{C}^{J+1}$ in the Hörmander class $\mathcal{S}\left(1+|z|_{\mathbb{C}^{J+1}}^{2}\right.$, $\left.\frac{|d z|_{\mathbb{C}^{J+1}}^{2}}{1+|z|_{\mathbb{C}^{J+1}}^{2}}\right)$. The functional calculus of Weyl $\varepsilon$-quantized elliptic operators in finite dimensions gives

$$
\forall s \in \mathbb{R},\left[\left(1+\left\langle z, A_{J} z\right\rangle\right)^{\mathrm{Wey} 1}\right]^{s} \geq\left(1-C_{J, s} \varepsilon\right)\left[\left(1+\left\langle z, A_{J} z\right\rangle\right)^{s}\right]^{\mathrm{Weyl}} .
$$

The finite-dimensional comparison of Wick and Weyl quantization, also gives

$$
\forall n \in \mathbb{N},\left[\left(1+\left\langle z, A_{J} z\right\rangle\right)^{n}\right]^{\text {Wick }} \geq\left(1-C_{J, n} \varepsilon\right)\left[\left(1+\left\langle z, A_{J} z\right\rangle\right)^{\text {Weyl }}\right]^{n} .
$$

From (4.3)(4.5) and the operator monotonicity of $B \rightarrow B^{t}$ for $t \in(0,1]$, we deduce

$$
\forall s \in \mathbb{R}, \quad(1+\mathrm{d} \Gamma(A))^{s} \geq\left(1-C_{J, s}^{\prime} \varepsilon\right)\left[\left(1+\left\langle z, A_{J} z\right\rangle\right)^{\mathrm{Weyl}}\right]^{s},
$$

and (4.4) gives

$$
\forall s \in \mathbb{R}, \quad(1+\mathrm{d} \Gamma(A))^{s} \geq\left(1-C_{J, s}^{\prime \prime} \varepsilon\right)\left[\left(1+\left\langle z, A_{J} z\right\rangle\right)^{s}\right]^{\text {Weyl }} .
$$

The definition of Wigner measures, recalled in Theorem 3.1, says

$$
\lim _{\varepsilon \rightarrow 0} \operatorname{Tr}\left[\varrho_{\varepsilon} b^{\mathrm{Weyl}}\right]=\int_{\mathcal{Z}_{0}} b(z) d \mu(z),
$$


for all $b \in \mathcal{S}_{\text {cyl }}\left(\mathcal{Z}_{0}\right)$, in particular the $b$ 's based on $\wp_{J} \mathcal{Z}_{0}$. Take now $s=\delta$ in (4.6). The a priori estimate

$\operatorname{Tr}\left[\varrho_{\varepsilon}\left[\left(1+\left\langle z, A_{J} z\right\rangle\right)^{\delta}\right]^{\mathrm{Weyl}}\right] \leq\left(1+C_{J, \delta} \varepsilon\right) \operatorname{Tr}\left[\varrho_{\varepsilon}(1+\mathrm{d} \Gamma(A))^{\delta}\right] \leq C_{\delta}\left(1+C_{J, \delta} \varepsilon\right)$, and the ellipticity of $\left(1+\left\langle z, A_{J} z\right\rangle\right)^{\delta}$ allows to extend the above convergence to any cylindrical $b=f \circ \wp_{J}$ with $f \in \mathcal{S}\left(\left(1+|z|_{\mathbb{C}^{J+1}}^{2}\right)^{\delta}, \frac{|d z|_{\mathbb{C}^{J+1}}^{2}}{1+|z|^{J}}\right)$. In particular, this leads to

$$
\begin{aligned}
C_{\delta}^{\prime} & \geq \limsup _{\varepsilon \rightarrow 0} \operatorname{Tr}\left[\varrho_{\varepsilon}(1+\mathrm{d} \Gamma(A))^{\delta}\right] \geq \lim _{\varepsilon \rightarrow 0} \operatorname{Tr}\left[\varrho_{\varepsilon}\left[\left(1+\left\langle z, A_{J} z\right\rangle\right)^{\delta}\right]^{\text {Weyl }}\right] \\
& =\int_{\mathcal{Z}_{0}}\left(1+\left\langle z, A_{J} z\right\rangle\right)^{\delta} d \mu(z) .
\end{aligned}
$$

Since $A=\sup _{J} A_{J}$ with $A_{J^{\prime}} \geq A_{J}$ for $J^{\prime} \geq J$, the monotone convergence implies

$$
C_{\delta}^{\prime} \geq \int_{\mathcal{Z}_{0}}(1+\langle z, A z\rangle)^{\delta} d \mu(z) \geq \int_{\mathcal{Z}_{0}}\langle z, A z\rangle^{\delta} d \mu(z) .
$$

Proposition 4.3. Within the framework of Theorem 1.1 with the assumption $\operatorname{Tr}[(\mathbf{N}+$ $\left.\left.H_{\varepsilon}^{0}\right)^{\delta} \varrho_{\varepsilon}\right] \leq C_{\delta}$ for $\delta \leq 6$, the measure $\mu_{t}$ satisfies the additional estimate

$$
\int_{\mathcal{Z}_{1}}|z|_{\mathcal{Z}_{1}}^{\frac{2 \delta}{3}} d \mu_{t}(z) \leq C_{\delta}
$$

for all times $t \in \mathbb{R}$.

Proof. The functional calculus of commuting operators implies

$$
c_{\delta}\left(1+S_{\varepsilon}\right)^{\delta / 3} \leq\left(1+\mathbf{N}+H_{\varepsilon}^{0}\right)^{\delta} \leq\left(1+S_{\varepsilon}\right)^{\delta} .
$$

Thus the initial state $\varrho_{\varepsilon}$, satisfies

$$
\operatorname{Tr}\left[\varrho_{\varepsilon}\left(1+S_{\varepsilon}\right)^{\delta / 3}\right] \leq C_{\delta}^{\prime} .
$$

From Proposition 2.3, we deduce

$$
e^{i \frac{t}{\varepsilon} H_{\varepsilon}}\left(1+S_{\varepsilon}\right)^{2} e^{-i \frac{t}{\varepsilon} H_{\varepsilon}} \leq C\left(1+S_{\varepsilon}\right)^{2} .
$$

Since $B \rightarrow B^{s}$ is operator monotone for $s \in(0,1]$, this implies

$$
e^{i \frac{t}{\varepsilon} H_{\varepsilon}}\left(1+S_{\varepsilon}\right)^{\delta / 3} e^{-i \frac{t}{\varepsilon} H_{\varepsilon}} \leq C^{\delta / 6}\left(1+S_{\varepsilon}\right)^{\delta / 3}
$$

as soon as $\frac{\delta}{3} \leq 2$. The inequality

$$
\operatorname{Tr}\left[\varrho_{\varepsilon}(t)(\mathrm{d} \Gamma(1-\Delta))^{\delta / 3}\right] \leq \operatorname{Tr}\left[\varrho_{\varepsilon}(t)\left(1+S_{\varepsilon}\right)^{\delta / 3}\right] \leq C_{\delta}^{\prime}
$$

and the previous Proposition 4.2 applied with $A=(1-\Delta)$, yields the result.

A more accurate version of this last result is given below by making use of the conservation of energy. 


\subsection{Convergence of moments and energy conservation}

For a family $\left(\varrho_{\varepsilon}\right)_{\varepsilon \in(0, \bar{\varepsilon})}$ of normal states with a single Wigner measure $\mu_{0}$ condition (4.2) is an important and non-trivial assumption. Indeed, we proved in [6] the following equivalence

$$
\begin{aligned}
(\forall \alpha & \left.\in \mathbb{N}, \lim _{\varepsilon \rightarrow 0} \operatorname{Tr}\left[\varrho_{\varepsilon} \mathbf{N}^{\alpha}\right]=\int_{\mathcal{Z}}|z|^{2 \alpha} d \mu_{0}(z)\right) \\
& \Leftrightarrow\left(\forall b \in \mathcal{P}_{\text {alg }}\left(\mathcal{Z}_{0}\right), \lim _{\varepsilon \rightarrow 0} \operatorname{Tr}\left[\varrho_{\varepsilon} b^{\text {Wick }}\right]=\int_{\mathcal{Z}} b(z) d \mu_{0}\right) .
\end{aligned}
$$

Hence condition (4.2), although it involves only the number operator, is exactly the one which leads to a good asymptotic behaviour of the reduced density matrices.

Proposition 4.4. Let $\left(\varrho_{\varepsilon}\right)_{\varepsilon \in(0, \bar{\varepsilon})}$ be a family of normal states on $\mathcal{H}$, satisfying the hypothesis of Theorem 1.1, with a single Wigner measure $\mu_{0}$. Assume $\operatorname{Tr}\left[\varrho_{\varepsilon} \mathbf{N}^{\alpha}\right] \leq$ $C_{\alpha}$ uniformly with respect to $\varepsilon \in(0, \bar{\varepsilon})$, for all $\alpha \in \mathbb{N}$. Then for every $\alpha \in \mathbb{N}$, the quantity

$$
\liminf _{\varepsilon \rightarrow 0} \operatorname{Tr}\left[\varrho_{\varepsilon}(t) \mathbf{N}^{\alpha}\right]-\int_{\mathcal{Z}_{0}}|z|_{\mathcal{Z}_{0}}^{2 \alpha} d \mu_{t}(z),
$$

does not depend on time when $\varrho_{\varepsilon}(t)=e^{-i \frac{t}{\varepsilon} H_{\varepsilon}} \varrho_{\varepsilon} e^{i \frac{t}{\varepsilon} H_{\varepsilon}}$ and $\mathcal{M}\left(\varrho_{\varepsilon}(t), \varepsilon \in(0, \bar{\varepsilon})\right)=$ $\left\{\mu_{t}\right\}$. Condition (4.2) is satisfied by $\left(\varrho_{\varepsilon}(t)\right)_{\varepsilon \in(0, \bar{\varepsilon})}$ and $\mu_{t}$, for all times $t \in \mathbb{R}$, as soon as it is true for one $t_{0} \in \mathbb{R}$.

Proof. According to Theorem 1.1, we know that $\mathcal{M}\left(\varrho_{\varepsilon}(t), \varepsilon \in(0, \bar{\varepsilon})\right)=\left\{\mu_{t}\right\}$ with $\mu_{t}=\Phi(t, 0)_{*} \mu_{0}$. Conservation of the $|\cdot|_{\mathcal{Z}_{0}}$-norm by the nonlinear flow $\Phi(t, 0)$ yields

$$
\int_{\mathcal{Z}_{0}}|z|_{\mathcal{Z}_{0}}^{2 \alpha} d \mu_{t}(z)=\int_{\mathcal{Z}_{0}}|z|_{\mathcal{Z}_{0}}^{2 \alpha} d \mu_{0}(z)
$$

for any $t \in \mathbb{R}$. On the other hand, $H_{\varepsilon}$ and $\mathbf{N}$ are strongly commuting self-adjoint operators therefore $\operatorname{Tr}\left[\varrho_{\varepsilon}(t) \mathbf{N}^{\alpha}\right]=\operatorname{Tr}\left[\varrho_{\varepsilon} \mathbf{N}^{\alpha}\right]$ for every $\alpha \in \mathbb{N}$.

Proposition 4.5. Let $\left(\varrho_{\varepsilon}\right)_{\varepsilon \in(0, \bar{\varepsilon})}$ be a family of normal states on $\mathcal{H}$ with a single Wigner measure $\mu_{0}$ satisfying the hypothesis of Theorem 1.1 with $\delta=2$ and condition (4.2). Then for any $t \in \mathbb{R}$

$$
\lim _{\varepsilon \rightarrow 0} \operatorname{Tr}\left[\varrho_{\varepsilon}(t) H_{\varepsilon}\right]=\int_{\mathcal{Z}_{1}} h(z, \bar{z}) d \mu_{t}(z) \quad \in(-\infty, \infty)
$$

where $\varrho_{\varepsilon}(t)=e^{-i \frac{t}{\varepsilon} H_{\varepsilon}} \varrho_{\varepsilon} e^{i \frac{t}{\varepsilon} H_{\varepsilon}}, \mu_{t}=\Phi(t, 0)_{*} \mu_{0}$ and $h(z, \bar{z})$ is the classical energy given in (1.2), and both sides of the identity do not depend on time.

Proof. With the energy conservation, it suffices to prove (4.8) for $t=0$. Let $\chi \in$ $C_{0}^{\infty}(\mathbb{R})$ such that $0 \leq \chi \leq 1, \chi(s)=1$ if $|s| \leq 1$ and $\chi(s)=0$ if $|s| \geq 2$. For 
$m \in \mathbb{N}^{*}$, set $\chi_{m}(x)=\chi\left(\frac{x}{m}\right)$. Let $B_{1}(z)$ and $B_{2}(z)$ be respectively the polynomial $\langle z,-\Delta z\rangle$ and $B_{2}(z)=V(z)=\frac{1}{2}\left\langle z^{\otimes 2}, V(x-y) z^{\otimes 2}\right\rangle$ well-defined for $z \in \mathcal{Z}_{1}$. Remember that although the kernels of $B_{1}$ and $B_{2}$ are unbounded operators their Wick quantization still have a meaning as densely defined operators on $\mathcal{H}$ (see Appendix B). Write for $j=1,2$

$$
\begin{aligned}
\left|\operatorname{Tr}\left[\varrho_{\varepsilon} B_{j}^{\text {Wick }}\right]-\int_{\mathcal{Z}_{1}} B_{j}(z) d \mu_{0}(z)\right| \leq & \left|\operatorname{Tr}\left[\varrho_{\varepsilon}\left(B_{j}^{\text {Wick }}-B_{j, m}^{\text {Wick }}\right)\right]\right| \\
& +\left|\operatorname{Tr}\left[\varrho_{\varepsilon} B_{j, m}^{\text {Wick }}\right]-\int_{\mathcal{Z}_{0}} B_{j, m}(z) d \mu_{0}(z)\right| \\
& +\left|\int_{\mathcal{Z}_{1}} B_{j, m}(z) d \mu_{0}(z)-\int_{\mathcal{Z}_{1}} B_{j}(z) d \mu_{0}(z)\right|,
\end{aligned}
$$

where $B_{1, m}(z)=\left\langle z,-\Delta\left[\chi_{m}(-\Delta)\right] z\right\rangle$ and $B_{2, m}(z)=\frac{1}{2}\left\langle z^{\otimes 2}, V(x-y)\left[\chi_{m}\left(-\Delta_{x}\right)\right] z^{\otimes 2}\right\rangle$. Observe that Lemma B.6 leads to

$$
\begin{array}{r}
\left\|(\mathrm{d} \Gamma(-\Delta)+\mathbf{N}+1)^{-1} \mathrm{~d} \Gamma\left(\Delta\left[\left(1-\chi_{m}\right)(-\Delta)\right]\right)(\mathrm{d} \Gamma(-\Delta)+\mathbf{N}+1)^{-1}\right\| \\
\leq\left\|\frac{-\Delta}{(1-\Delta)^{2}}\left[\left(1-\chi_{m}\right)(-\Delta)\right]\right\| \stackrel{m \rightarrow \infty}{\rightarrow} 0
\end{array}
$$

and

$$
\begin{aligned}
\|(\mathrm{d} \Gamma(-\Delta)+\mathbf{N}+1)^{-1}\left(B_{2}^{\text {Wick }}\right. & \left.-B_{2, m}^{\text {Wick }}\right)(\mathrm{d} \Gamma(-\Delta)+\mathbf{N}+1)^{-1} \| \\
& \leq C_{V}\left\|(1-\Delta)^{-1 / 2}\left(1-\chi_{m}\right)(-\Delta)\right\| \stackrel{m \rightarrow \infty}{\rightarrow} 0 .
\end{aligned}
$$

Therefore the right-hand side (4.9) tends to 0 when $m \rightarrow \infty$ thanks to the regularity of $\varrho_{\varepsilon}$. Now, since $B_{j, m}, j=1,2$ belong to $\mathcal{P}_{\text {alg }}\left(\mathcal{Z}_{0}\right)$ then by the statement (4.7), proved in [6, Proposition 2.12], the right-hand side (4.10) converges to 0 when $\varepsilon \rightarrow$ 0 . Further, by the dominated convergence theorem and with the help of Lemma 3.13 , the right-hand side (4.11) vanishes as $m \rightarrow \infty$. Hence a $\delta / 3$-argument gives

$$
\lim _{\varepsilon \rightarrow 0} \operatorname{Tr}\left[\varrho_{\varepsilon} B_{j}^{\text {Wick }}\right]=\int_{\mathcal{Z}_{1}} B_{j}(z) d \mu_{0}(z) \quad \text { for } \quad j=1,2 .
$$

Thus (4.8) is proved.

\subsection{Examples}

We give here two examples, other can be found in our previous articles [4-6]. The first one recalls that the transport of the Wigner measure takes into account some correlations. The second one is about the mean field dynamics of states, which do not satisfy (4.2) and makes a connection with Bose-Einstein condensation. 


\subsection{Deformed tori}

For two elements $\psi_{1}, \psi_{2} \in \mathcal{Z}_{1} \subset \mathcal{Z}_{0}$ such that $\left\|\psi_{1}\right\|=\left\|\psi_{2}\right\|=1$ and $\left\langle\psi_{1}, \psi_{2}\right\rangle=$ 0 , the space $\mathcal{Z}_{0}$ can be decomposed into

$$
\mathcal{Z}_{0}=\mathbb{C} \psi_{1} \stackrel{\perp}{\oplus} \mathbb{C} \psi_{1} \stackrel{\perp}{\oplus} \psi^{\perp} .
$$

This decomposition is second-quantized into the Hilbert tensor product

$$
\mathcal{H}=\Gamma_{s}\left(\mathcal{Z}_{0}\right)=\Gamma_{s}\left(\mathbb{C} \psi_{1}\right) \otimes \Gamma_{s}\left(\mathbb{C} \psi_{2}\right) \otimes \Gamma_{s}\left(\psi^{\perp}\right),
$$

which allows an analysis by separating the variables. The number observable is now

$$
\mathbf{N}=\left(\mathbf{N}_{1} \otimes \mathbb{1} \otimes \mathbb{1}\right) \oplus\left(\mathbb{1} \otimes \mathbf{N}_{2} \otimes \mathbb{l}\right) \oplus\left(\mathbb{1} \otimes \mathbb{1} \otimes \mathbf{N}^{\prime}\right),
$$

simply written as $\mathbf{N}=\mathbf{N}_{1}+\mathbf{N}_{2}+\mathbf{N}^{\prime}$, where $\mathbf{N}_{1}, \mathbf{N}_{2}$ and $\mathbf{N}^{\prime}$ are respectively the number operators on $\Gamma_{s}\left(\mathbb{C} \psi_{1}\right), \Gamma_{s}\left(\mathbb{C} \psi_{2}\right)$ and $\Gamma_{s}\left(\psi^{\perp}\right)$. Consider in this decomposition, the state

$$
\varrho_{\varepsilon}=\varrho_{\varepsilon}^{1} \otimes \varrho_{\varepsilon}^{2} \otimes\left(\left|\Omega^{\prime}\right\rangle\left\langle\Omega^{\prime}\right|\right)
$$

where $\left|\Omega^{\prime}\right\rangle$ is the vacuum state of $\Gamma_{S}\left(\psi^{\perp}\right)$ and

$$
\begin{aligned}
& \varrho_{\varepsilon}^{1}=\left|\psi_{1}^{\otimes n_{1}}\right\rangle\left\langle\psi_{1}^{\otimes n_{1}}\left|\quad, \quad \varrho_{\varepsilon}^{2}=\right| \psi_{2}^{\otimes n_{2}}\right\rangle\left\langle\psi_{2}^{\otimes n_{2}}\right|, \\
& \text { with } \lim _{\varepsilon \rightarrow 0} \varepsilon n_{1}=\lim _{\varepsilon \rightarrow 0} \varepsilon n_{2}=\frac{1}{2} .
\end{aligned}
$$

In $\mathcal{H}=\Gamma_{s}\left(\mathcal{Z}_{0}\right)$, this state is explicitly written (see [6]) as

$$
\varrho_{\varepsilon}=\left|\psi^{\vee\left(n_{1}, n_{2}\right)}\right\rangle\left\langle\psi^{\vee\left(n_{1}, n_{2}\right)}\right|
$$

with $\psi^{\vee\left(n_{1}, n_{2}\right)}=\frac{1}{\sqrt{\varepsilon^{n_{1}+n_{2} n_{1} ! n_{2} !}}} \overbrace{a^{*}\left(\psi_{1}\right) \ldots a^{*}\left(\psi_{1}\right)}^{n_{1} \text { times }} \overbrace{a^{*}\left(\psi_{2}\right) \ldots a^{*}\left(\psi_{2}\right)}^{n_{2} \text { times }}|\Omega\rangle$.

The state satisfies

$$
\lim _{\varepsilon \rightarrow 0} \operatorname{Tr}\left[\mathbf{N}^{k} \varrho_{\varepsilon}\right]=\left(\frac{1}{2}+\frac{1}{2}\right)^{k}=1,
$$

owing to $\mathbf{N}=\mathbf{N}_{1}+\mathbf{N}_{2}+\mathbf{N}^{\prime}$. Moreover, with (4.12) and (4.13), $\mathbf{N}+H_{\varepsilon}^{0}=\mathrm{d} \Gamma(1-\Delta)$ and the help of Wick calculus, it also fulfills

$$
\lim _{\varepsilon \rightarrow 0} \operatorname{Tr}\left[\left(\mathbf{N}+H_{\varepsilon}^{0}\right) \varrho_{\varepsilon}\right]=\frac{\left|\psi_{1}\right|_{\mathcal{Z}_{1}}^{2}+\left|\psi_{2}\right|_{\mathcal{Z}_{1}}^{2}}{2} .
$$

Meanwhile the separation of variables allows to compute explicitly the (it is unique) Wigner measure of $\left(\varrho_{\varepsilon}\right)_{\varepsilon \in(0, \bar{\varepsilon})}$

$$
\mu_{0}=\delta_{\frac{\sqrt{2}}{2} \psi_{1}}^{S^{1}} \otimes \delta_{\frac{\sqrt{2}}{2} \psi_{1}}^{S^{1}} \otimes \delta_{0} \quad \text { on } \quad \mathcal{Z}_{1}=\left(\mathbb{C} \psi_{1}\right) \times\left(\mathbb{C} \psi_{2}\right) \times \psi^{\perp},
$$

with $\delta_{u}^{S^{1}}=\frac{1}{2 \pi} \int_{0}^{2 \pi} \delta_{e^{i \theta} u} d \theta$. 
We get

$$
\int_{\mathcal{Z}_{1}}|z|^{2 k} d \mu_{0}(z)=\int_{\mathcal{Z}_{1}}\left(\left|z_{1}\right|^{2}+\left|z_{2}\right|^{2}+\left|z^{\prime}\right|^{2}\right)^{k} d \mu_{0}(z)=1=\lim _{\varepsilon \rightarrow \infty} \operatorname{Tr}\left[\mathbf{N}^{k} \varrho_{\varepsilon}\right] .
$$

Hence all the assumptions of Theorem 1.1 and Theorem 4.1 are fulfilled. This measure is carried by a torus in $\mathcal{Z}_{1}$ better described by using an other orthonormal basis of $\mathbb{C} \psi_{1} \oplus \mathbb{C} \psi_{2}$ :

$$
\begin{aligned}
& \psi_{0}=\frac{\sqrt{2}}{2}\left(\psi_{1}+\psi_{2}\right), \quad \psi_{\frac{\pi}{2}}=i \frac{\sqrt{2}}{2}\left(\psi_{1}-\psi_{2}\right), \\
& \psi_{\varphi}=\cos (\varphi) \psi_{0}+\sin (\varphi) \psi \frac{\pi}{2}, \\
& \frac{\sqrt{2}}{2}\left(e^{i \theta} \psi_{1}+e^{i \theta^{\prime}} \psi_{2}\right)=e^{i \frac{\theta+\theta^{\prime}}{2}} \psi_{\frac{\theta-\theta^{\prime}}{2}} \\
& \mu_{0}=\frac{1}{2 \pi} \int_{0}^{2 \pi} \delta_{\psi_{\varphi}}^{S^{1}} d \varphi .
\end{aligned}
$$

Two elements $e^{i \theta} \psi_{\varphi}$ and $e^{i \theta^{\prime}} \psi_{\varphi^{\prime}}$ in the support of $\mu_{0}$ are equal when

$$
\left(\theta^{\prime}=\theta \text { and } \varphi^{\prime}=\varphi\right) \quad \text { or } \quad\left(\theta^{\prime}=\theta+\pi \text { and } \varphi^{\prime}=\varphi+\pi\right) \text {. }
$$

Hence a one-to-one parametrization of the torus can be done by $\varphi \in[0,2 \pi)$ and $\theta \in[\varphi, \varphi+\pi)$.

Let $\psi_{\varphi}(t)=\Phi(t, 0) \psi_{\varphi}$, be the solution to the Hartree equation

$$
\left\{\begin{array}{l}
i \partial_{t} \psi_{\varphi}(t)=-\Delta \psi_{\varphi}(t)+\left(V *\left|\psi_{\varphi}(t)\right|^{2}\right) \psi_{\varphi}(t) \\
\psi_{\varphi}(t=0)=\psi_{\varphi}=e^{i \frac{\pi}{4}} \cos (\varphi) \psi_{1}+e^{-i \frac{\pi}{4}} \sin (\varphi) \psi_{2}
\end{array}\right.
$$

The gauge invariance of the equation says that for any $\theta \in[0,2 \pi], e^{i \theta} \psi_{\varphi}(t)=$ $\Phi(t, 0)\left[e^{i \theta} \psi_{\varphi}\right]$. By applying the result of Theorem 1.1 and Theorem 4.1 we get

$$
\begin{aligned}
& \mu_{t}=\frac{1}{2 \pi} \int_{0}^{2 \pi} \delta_{\psi_{\varphi}(t)}^{S^{1}} d \varphi=\frac{1}{4 \pi^{2}} \int_{0}^{2 \pi} \int_{0}^{2 \pi} \delta_{e^{i \theta} \psi_{\varphi}(t)} d \varphi d \theta \\
& \forall p \in \mathbb{N}, \quad \lim _{\varepsilon \rightarrow 0} \gamma_{\varepsilon}^{(p)}(t)=\frac{1}{2 \pi} \int_{0}^{2 \pi}\left|\left[\psi_{\varphi}(t)\right]^{\otimes p}\right\rangle\left\langle\left[\psi_{\varphi}(t)\right]^{\otimes p}\right| d \varphi .
\end{aligned}
$$

Since the Hartree flow is nonlinear, the complete hierarchy of reduced density matrices have to be taken into account if one wants to write evolution equation for them. More simply, they can be computed after solving an autonomous equation for the Wigner measure. Due to the nonlinear term the dynamics of correlations is by far non-trivial. This can also be thought geometrically: The initial measure is initially supported by a torus which lies in a 2-dimensional complex vector space 

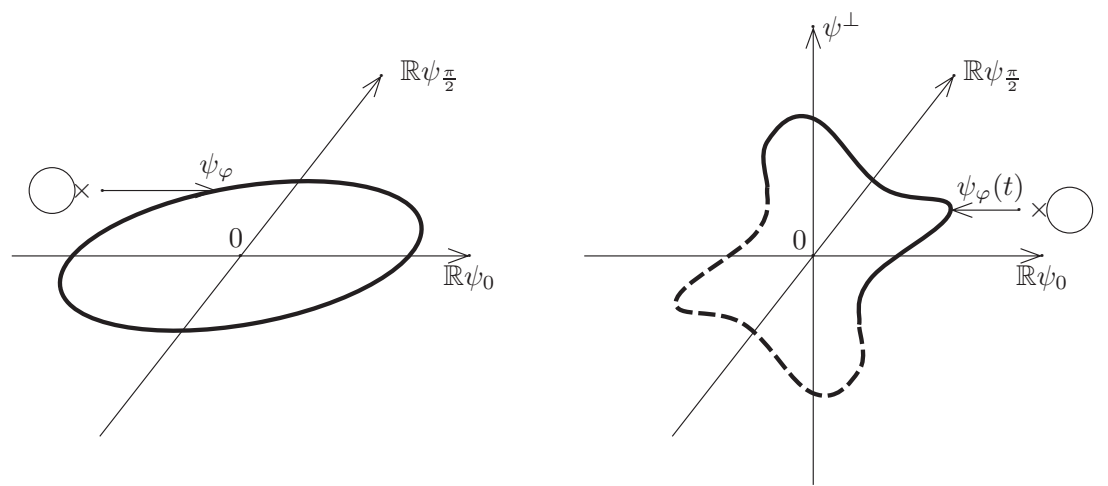

Figure 4.1. Evolution of the measure initially carried by a torus in $\mathbb{C} \psi_{0} \oplus \mathbb{C} \psi_{\frac{\pi}{2}}$. The complex gauge parameter $e^{i \theta}$ is represented by the small circle.

(think of the circle in the plane $\mathbb{R} \psi_{0} \oplus \mathbb{R} \psi_{\frac{\pi}{2}}$ ); along the time evolution, the measure $\mu_{t}$ is still carried by a torus in $\mathcal{Z}_{1}$, which nevertheless, is a priori not embedded in any finite-dimensional subspace. In Figure 4.1, the deformed torus for time $t \neq 0$, has to be imagined in the infinite-dimensional phase-space $\mathcal{Z}_{1} \subset \mathcal{Z}_{0}$. Contrary to the picture, there might be no intersection with the real plane $\mathbb{R} \psi_{0} \oplus \mathbb{R} \psi_{\frac{\pi}{2}}$.

This discussion can also be extended to higher dimensional tori after taking a finite (or countable) orthornormal family $\left(\psi_{n}\right)_{1 \leq n \leq N}$ for building the initial states $\varrho_{\varepsilon}$ with a measure $\prod_{j=1}^{N} \delta_{\lambda_{j} \psi_{j}}^{S^{1}}($ see [6]).

\subsection{Propagation without the convergence of moments}

In [4] we considered the thermodynamic limit of a free Bose gas on a torus with the one particle energy given by $-\Delta$. We showed that in the regime which may exhibit a Bose condensation, condition (4.2) fails and illustrates what we called a dimensional defect of compactness, in opposition to the phase space or microlocal defect of compactness (see [28,61]). Others examples were given. In [6] the propagation result for bounded interactions but without any compactness condition, cannot be applied for such initial states. With Theorem 1.1 the propagation holds for this kind of initial states. Since our analysis is valid on $\mathbb{R}^{d}$ the analysis for the torus does not apply directly and we adapt the presentation of the Bose-Einstein condensation.

Moreover the dimensional defect of compactness which plays with all the directions of the phase-space $\mathcal{Z}_{0}=L^{2}\left(\mathbb{R}^{d}\right)$, can be geometrically thought in the one particle phase-space $T^{*} \mathbb{R}^{d}$. Condition (1.4), which leads to estimates of $\int_{\mathcal{Z}_{0}}|z|_{H^{1}}^{2 \delta} d \mu$, suggests that the dimensional defect of compactness is due to mass going to $\infty$ in the position variable rather than in the momentum variable, in $T^{*} \mathbb{R}^{d}$. The mean field limit that we consider here, can be tested by using the harmonic oscillator Hamiltonian $A=-\partial_{x}^{2}+\frac{x^{2}}{4}-\frac{d}{4}$. The motivated reader will then see that the dimensional defect of compactness $\varrho_{\varepsilon}$ is incompatible with condition (1.4). 
We work in dimension $d \geq 2$. Let $e_{0}$ be an $L^{2}$-normalized $\mathcal{C}^{\infty}$ function supported in the hypercube $\left(-\frac{1}{2}, \frac{1}{2}\right)^{d}$ and set

$$
\forall k \in \mathbb{N}^{d}, \quad e_{k}(x)=e_{0}(x-k) .
$$

The family $\left(e_{k}\right)_{k \in \mathbb{N}}$ is orthonormal in $\mathcal{Z}_{0}=L^{2}\left(\mathbb{R}^{d}\right)$. The spanned Hilbert subspace and the corresponding orthogonal projection are respectively denoted by $\mathcal{Z}_{e}$ and $\Pi_{e}, \operatorname{Ran} \Pi_{e}=\mathcal{Z}_{e}$. Note that

$$
\Pi_{e}(-\Delta) \Pi_{e}=\left(\int_{\mathbb{R}}\left|\nabla e_{0}\right|^{2}\right) \Pi_{e} .
$$

Consider now the self-adjoint operator defined on $\mathcal{Z}_{0}=L^{2}\left(\mathbb{R}^{d}\right)$ by

$$
A=\sum_{k \in \mathbb{N}^{d}}|k|\left|e_{k}\right\rangle\left\langle e_{k}|, \quad| k\right|=\sum_{j=1}^{d} k_{j},
$$

which restricted to $\mathcal{Z}_{e}$ is unitarily equivalent to the harmonic oscillator Hamiltonian $A=-\partial_{x}^{2}+\frac{x^{2}}{4}-\frac{d}{4}$ on $\mathbb{R}^{d}$. We use the tensor decomposition

$$
\begin{aligned}
& \mathcal{Z}_{0}=\mathcal{Z}_{e} \stackrel{\perp}{\oplus} \mathcal{Z}_{e}^{\perp} \quad, \quad \mathcal{H}=\Gamma_{s}\left(\mathcal{Z}_{0}\right)=\Gamma_{s}\left(\mathcal{Z}_{e}\right) \otimes \Gamma_{s}\left(\mathcal{Z}_{e}^{\perp}\right), \\
& \mathbf{N}=\mathbf{N}_{e} \otimes \mathbb{1}+\mathbb{1} \otimes \mathbf{N}_{e}^{\perp}=\mathbf{N}_{e}+\mathbf{N}_{e}^{\perp} \quad, \quad|\Omega\rangle=\left|\Omega_{e}\right\rangle \otimes\left|\Omega_{e}^{\perp}\right\rangle, \\
& \forall B_{e} \in \mathcal{L}\left(\mathcal{Z}_{e}\right),\left\|B_{e}\right\| \leq 1, \quad \Gamma\left(B_{e}\right) \otimes\left(\left|\Omega_{e}^{\perp}\right\rangle\left\langle\Omega_{e}^{\perp}\right|\right)=\Gamma\left(\Pi_{e} B_{e} \Pi_{e}\right), \\
& \forall B \in \mathcal{L}\left(\mathcal{Z}_{0}\right),\|B\| \leq 1, \quad \Gamma\left(\Pi_{e} B \Pi_{e}\right)=\Gamma\left(\Pi_{e} \circ B \mid \mathcal{Z}_{e}\right) \otimes\left(\left|\Omega_{e}^{\perp}\right\rangle\left\langle\Omega_{e}^{\perp}\right|\right) .
\end{aligned}
$$

In particular the last relation with $B=e^{i \varepsilon t \Delta}$ differentiated at time $t=0$ gives

$$
\Gamma\left(\Pi_{e}\right) \mathrm{d} \Gamma(-\Delta) \Gamma\left(\Pi_{e}\right)=\left(\int_{\mathbb{R}^{d}}\left|\nabla e_{0}\right|^{2}\right) \mathbf{N}_{e}
$$

Consider on $\mathcal{H}$, the $\varepsilon$-dependent gauge invariant (tensorized) quasi-free state

$$
\begin{aligned}
& \varrho_{\varepsilon}=\frac{1}{\operatorname{Tr}\left[\Gamma\left(\Pi_{e} e^{-\beta_{\varepsilon}\left(A-\mu_{\varepsilon}\right)} \Pi_{e}\right)\right]} \Gamma\left(\Pi_{e} e^{-\beta_{\varepsilon}\left(A-\mu_{\varepsilon}\right)} \Pi_{e}\right) \\
& =\frac{1}{\operatorname{Tr}\left[\Gamma\left(\Pi_{e} Z_{\varepsilon} e^{-\beta_{\varepsilon} A} \Pi_{e}\right)\right]} \Gamma\left(\Pi_{e} Z_{\varepsilon} e^{-\beta_{\varepsilon} A} \Pi_{e}\right)
\end{aligned}
$$

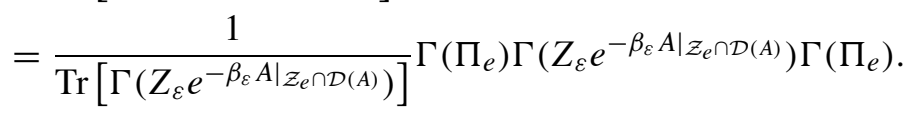

The chemical potential $\mu_{\varepsilon}$ is negative of order $\varepsilon^{1-1 / d}$ and the temperature is large according to

$$
Z_{\varepsilon}=e^{\beta_{\varepsilon} \mu_{\varepsilon}}=1-\frac{\varepsilon}{v_{C}} \quad, \quad \beta_{\varepsilon}=\varepsilon^{1 / d} .
$$


With the $\varepsilon$-dependent definition of $a(f), a^{*}(f),\left[a(g), a^{*}(f)\right]=\varepsilon\langle g, f\rangle$, and $W(f)$, this quasi-free state is characterized by the two-point function

$$
\begin{aligned}
& \left.\operatorname{Tr}\left[\varrho_{\varepsilon} a^{*}(f) a(g)\right)\right]=\varepsilon\left\langle\Pi_{e} g, Z_{\varepsilon} e^{-\beta_{\varepsilon} A_{\varepsilon}}\left(1-Z_{\varepsilon} e^{-\beta_{\varepsilon} A_{\varepsilon}}\right)^{-1} \Pi_{e} f\right\rangle \text { or } \\
& \operatorname{Tr}\left[\varrho_{\varepsilon} W(f)\right]=\exp \left[-\varepsilon\left\langle\Pi_{e} f,\left(1+Z_{\varepsilon} e^{-\beta_{\varepsilon} A_{\varepsilon}}\right)\left(1-Z_{\varepsilon} e^{-\beta_{\varepsilon} A_{\varepsilon}}\right)^{-1} \Pi_{e} f\right\rangle / 4\right] .
\end{aligned}
$$

In particular the total number (multiplied by $\varepsilon$ ) is given by

$$
\begin{aligned}
\operatorname{Tr}\left[\varrho_{\varepsilon} \mathbf{N}\right] & =\operatorname{Tr}\left[\varrho_{\varepsilon} \mathbf{N}_{e}\right] \sum_{k \in \mathbb{N}^{d}} \operatorname{Tr}\left[\varrho_{\varepsilon} a^{*}\left(e_{k}\right) a\left(e_{k}\right)\right] \\
& =v_{C}+v+r(\varepsilon) \quad \text { with } \lim _{\varepsilon \rightarrow 0} r(\varepsilon)=0, \text { and } \\
v & =\int_{\mathbb{R}^{d}} \frac{e^{-|u|}}{1-e^{-|u|}} d u=\left|S^{d-1}\right| \int_{0}^{+\infty} \frac{e^{-t}}{1-e^{-t}} t^{d-1} d t(d \geq 2) .
\end{aligned}
$$

We deduce

$$
\begin{aligned}
& \lim _{\varepsilon \rightarrow 0} \operatorname{Tr}\left[\varrho_{\varepsilon} \mathbf{N}\right]=v_{C}+v \\
& \lim _{\varepsilon \rightarrow 0} \operatorname{Tr}\left[\varrho_{\varepsilon} H_{\varepsilon}^{0}\right]=\lim _{\varepsilon \rightarrow 0} \operatorname{Tr}\left[\varrho_{\varepsilon} \Gamma\left(\Pi_{e}\right) \mathrm{d} \Gamma(-\Delta) \Gamma\left(\Pi_{e}\right)\right]=\left(\int_{\mathbb{R}^{d}}\left|\nabla e_{0}\right|^{2}\right)\left(v_{C}+v\right),
\end{aligned}
$$

and condition (1.4) of Theorem 1.1 is satisfied.

Actually $v_{C}>0$ corresponds, in the analysis of the free Bose gas (see [4]), to the density associated with the condensate phase. In the scaling that we consider, it is the other part which produces the dimensional defect of compactness. Let us compute the Wigner measure, by considering the limit of $\operatorname{Tr}\left[\varrho_{\varepsilon} W(\sqrt{2} \pi f)\right]$ as $\varepsilon \rightarrow 0$. With

$$
f=\sum_{k \in \mathbb{N}^{d}} f_{k} e_{k}+f^{\perp}, \quad|f|^{2}=\sum_{k \in \mathbb{N}^{d}}\left|f_{k}\right|^{2}+\left|f^{\perp}\right|^{2}=\left|\Pi_{e} f\right|^{2}+\left|f^{\perp}\right|^{2},
$$

expression (4.16) gives

$$
\begin{aligned}
\operatorname{Tr}\left[\varrho^{\varepsilon} W(\sqrt{2} \pi f)\right] & =e^{-\varepsilon \pi^{2}\left|\Pi_{e} f\right|^{2} / 2} \times \exp \left[-\varepsilon \pi^{2} \sum_{k \in \mathbb{N}^{d}}\left|f_{k}\right|^{2} \frac{Z_{\varepsilon} e^{-\varepsilon^{1 / d}|k|}}{\left(1-Z_{\varepsilon} e^{-\varepsilon^{1 / d}|k|}\right)}\right] \\
& \stackrel{\varepsilon \rightarrow 0}{\rightarrow} e^{-\pi^{2} \nu_{C}\left|f_{0}\right|^{2}}
\end{aligned}
$$

The family $\left(\varrho_{\varepsilon}\right)_{\varepsilon \in(0, \bar{\varepsilon})}$ admits the unique Wigner measure

$$
\mu_{0}=\frac{e^{-\frac{\left|z_{0}\right|^{2}}{v_{C}}}}{\pi v_{C}} \otimes \delta_{0} \quad \text { on } \mathcal{Z}_{0}=\left(\mathbb{C} e_{0}\right) \times e_{0}^{\perp},
$$


which is carried by $\mathbb{C} e_{0} \subset \mathcal{Z}_{1}$ and which can also be written as

$$
\mu_{0}=\int_{\mathbb{C} e_{0}} \frac{e^{-\frac{|z|^{2}}{\nu_{C}}}}{\pi v_{C}} \delta_{z e_{0}} L_{\mathbb{C} e_{0}}(d z)=\int_{0}^{+\infty} \frac{e^{-\frac{u}{\nu_{C}}}}{v_{C}} \delta_{\sqrt{u} e_{0}}^{S^{1}} d u .
$$

In particular, we get

$$
\begin{aligned}
& \int_{\mathcal{Z}_{1}}|z|_{\mathcal{Z}_{0}}^{2} d \mu_{0}(z)=v_{C}<v_{C}+v=\lim _{\varepsilon \rightarrow 0} \operatorname{Tr}\left[\varrho_{\varepsilon} \mathbf{N}\right] \\
& \text { and } \int_{\mathcal{Z}_{1}}|z|_{\mathcal{Z}_{1}}^{2} d \mu_{0}(z)=v_{C}\left(\int_{\mathbb{R}^{d}}\left|\nabla e_{0}\right|^{2}\right)<\left(v_{C}+v\right)\left(\int_{\mathbb{R}^{d}}\left|\nabla e_{0}\right|^{2}\right) \\
& =\lim _{\varepsilon \rightarrow 0} \operatorname{Tr}\left[\varrho_{\varepsilon} \mathrm{d} \Gamma(1-\Delta)\right] \text {, }
\end{aligned}
$$

and condition (4.2) does not hold. Even at time $t=0$, no formula is available for the reduced density matrices in terms of the Wigner measure. Nevertheless the timedependent Wigner measure of $\varrho_{\varepsilon}(t)=e^{-i \frac{t}{\varepsilon} H_{\varepsilon}} \varrho_{\varepsilon} e^{i \frac{t}{\varepsilon} H_{\varepsilon}}$ is given by Theorem 1.1, since condition (1.4) is verified. Consider the solutions to the Hartree initial value problems

$$
\left\{\begin{array}{l}
i \partial_{t} \psi_{u}=-\Delta \psi_{u}+\left(V *\left|\psi_{u}\right|^{2}\right) \psi_{u} \\
\psi_{u}(t=0)=\sqrt{u} e_{0}, \quad u \in(0,+\infty)
\end{array}\right.
$$

Then the Wigner measure of $\varrho_{\varepsilon}(t)=e^{-i \frac{t}{\varepsilon} H_{\varepsilon}} \varrho_{\varepsilon} e^{i \frac{t}{\varepsilon} H_{\varepsilon}}$ is given by

$$
\mu_{t}=\int_{0}^{+\infty} \frac{e^{-\frac{u}{v_{C}}}}{v_{C}} \delta_{\psi_{u}(t)}^{S^{1}} d u
$$

Again like in the example of the previous section, the measure $\mu_{t}$ is carried by surface containing 0 and topologically equivalent to $\mathbb{C}$, but this 2 -dimensional surface does not remain a priori in any finite-dimensional subspace of $\mathcal{Z}_{1}$ for $t \neq 0$.

\subsection{About the classical mean field problem}

The classical analogue of our analysis is the derivation of the Vlasov equation

$$
\left\{\begin{array}{l}
\partial_{t} f+v \cdot \partial_{x} f-\frac{1}{m}\left(\partial_{x} V_{f}(x, t)\right) . \partial_{v} f=0 \\
f(t, x, v)=f_{0}(x, v) \\
V_{f}(x, t)=V * \varrho_{f}(x, t) \quad, \quad \varrho_{f}(x, t)=\int_{\mathbb{R}^{d}} f(x, v, t) d v
\end{array}\right.
$$

where $f(x, v, t)$ represents the particle density in the 1 -particle phase space $\mathbb{R}_{x, v}^{2 d}$, from the classical Hamilton many body system

$$
\left\{\begin{array}{l}
\dot{x}_{i}=v_{i}, \\
\dot{v}_{i}=-\frac{1}{m N}\left(\sum_{j=1}^{N} \partial_{x_{i}} V\left(x_{i}-x_{j}\right)\right)
\end{array}, \quad i=1, \ldots, N,\right.
$$


in the limit $N \rightarrow \infty$. This problems is still open for singular potential and C. Villani, in a recent survey article about the Landau damping [52] quotes the work of Hauray-Jabin [36] as the most advanced one in this direction. It works for a potential such that $|\nabla V|=\mathcal{O}\left(|x|^{-s}\right), s \in(0,1)$, and does not include the Coulomb interaction.

Indirectly, our result justifies the mean field model up to Coulomb interaction in dimension $d=3$. In [49] and more recently [2], the Vlasov equation is proved to be the semiclassical limit of the semiclassical Hartree equation. This means that there are two "semiclassical" limits, one in the phase-space $L^{2}\left(\mathbb{R}^{d} ; \mathbb{C}\right)$ with the small parameter $1 / N$, another one on the phase-space $T^{*} \mathbb{R}^{d} \sim \mathbb{R}^{2 d}$ for the one particle nonlinear problem. This double asymptotic regime is well presented in $[25,27,35]$.

A possible strategy for directly deriving the classical mean field limit from the classical many body problem consists in adapting our approach by, as usual, replacing traces by integrals. For information, we refer the reader to the presentation [18] by J. Derezinski of the classical analogue of second quantization. Of course classical mechanics, although living in the commutative world, is often more singular than quantum mechanics, from the analysis point of view. With the Coulomb interaction, the Kustaanheimo-Stiefel desingularization of the Hamiltonian flow may be useful (see a.e. [15,38,41,43,46]).

\section{Appendices}

\section{A. Commuting self-adjoint operators on a graded Hilbert space}

We briefly study the general structure of self-adjoint operators on a graded Hilbert space. Properties collected in this section are useful for the analysis of the quantum Hamiltonian (1.1). In this appendix, the small parameter is not required and we work with $\varepsilon=1$.

Remember that a graded Hilbert space $\mathcal{H}$ is a direct sum of Hilbert spaces $\mathcal{H}_{n}, n \in \mathbb{N}$, of the form

$$
\mathcal{H}=\bigoplus_{n=0}^{\infty} \mathcal{H}_{n} .
$$

Let $\left(A_{n}\right)_{n \in \mathbb{N}}$ be a sequence of self-adjoint operators where each $A_{n}$ acts on $\mathcal{H}_{n}$. We define the operator

$$
\begin{aligned}
\mathcal{D}(A) & =\left\{\Psi \in \mathcal{H}: \sum_{n=0}^{\infty}\left\|A_{n} \Psi^{(n)}\right\|_{\mathcal{H}_{n}}^{2}<\infty\right\}, \\
A \Psi & =\sum_{n=0}^{\infty} A_{n} \Psi^{(n)}, \text { for all } \Psi \in \mathcal{D}(A) .
\end{aligned}
$$


Taking in particular $A_{n}=n \mathbb{1}_{\mathcal{H}_{n}}$ for $n \in \mathbb{N}$, we obtain the number operator

$$
N=\sum_{n=0}^{\infty} n \mathbb{1}_{\mathcal{H}_{n}} .
$$

We say that two self-adjoint operators $B$ and $C$ on a Hilbert space strongly commute if their spectral projections mutually commute. This is equivalent to the commutation of their resolvents for some $z \in \mathbb{C} \backslash \mathbb{R}$ and also to the commutation of their associated unitary groups. More precisely, $B$ and $C$ strongly commute if and only if for all $t, s \in \mathbb{R}$

$$
e^{i t C} e^{i s B}=e^{i s B} e^{i t C} .
$$

Proposition A.1. Let $A$ and $N$ be the operators given by (A.1) and (A.2). The following assertions hold:

(i) A and $N$ are self-adjoint;

(ii) For any bounded Borel function on $\mathbb{R}$

$$
f(A)=\sum_{n=0}^{\infty} f\left(A_{n}\right)
$$

(iii) The operators $A$ and $N$ strongly commute;

(iv) If $\mathcal{D}_{n}$ is a core for $A_{n}$ for each $n \in \mathbb{N}$ then $\oplus_{n \in \mathbb{N}}^{\text {alg }} \mathcal{D}_{n}$ is a core for $A$;

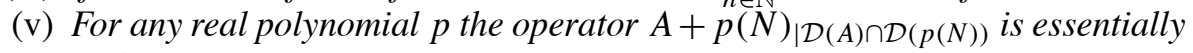
self-adjoint and

$$
\overline{A+p(N)_{\mid \mathcal{D}(A) \cap \mathcal{D}(p(N))}}=\sum_{n=0}^{\infty} A_{n}+p(n) \mathbb{1}_{\mathcal{H}_{n}} .
$$

Proof. (i) Clearly, $A$ is a densely defined operator. It is also symmetric, since for any $\Psi, \Phi \in \mathcal{D}(A)$

$$
\langle\Phi, A \Psi\rangle_{\mathcal{H}}=\sum_{n=0}^{\infty}\left\langle\Phi^{(n)}, A_{n} \Psi^{(n)}\right\rangle_{\mathcal{H}_{n}}=\sum_{n=0}^{\infty}\left\langle A_{n} \Phi^{(n)}, \Psi^{(n)}\right\rangle_{\mathcal{H}_{n}}=\langle A \Phi, \Psi\rangle_{\mathcal{H}}
$$

For any $\Psi \in \mathcal{D}(A)$ and $\Phi \in \mathcal{D}\left(A^{*}\right)$,

$$
\left\langle A^{*} \Phi, \Psi\right\rangle=\sum_{n=0}^{\infty}\left\langle\Phi^{(n)}, A_{n} \Psi^{(n)}\right\rangle_{\mathcal{H}_{n}} .
$$

Hence the following inequality holds:

$$
\left|\sum_{n=0}^{\infty}\left\langle\Phi^{(n)}, A_{n} \Psi^{(n)}\right\rangle_{\mathcal{H}_{n}}\right| \leq\left\|A^{*} \Phi\right\|_{\mathcal{H}}\|\Psi\|_{\mathcal{H}}
$$


Taking any $\Psi^{(n)} \in \mathcal{D}\left(A_{n}\right)$, this means that $\Phi^{(n)} \in \mathcal{D}\left(A_{n}^{*}\right)=\mathcal{D}\left(A_{n}^{*}\right)=\mathcal{D}\left(A_{n}\right)$. Extension to any $\Psi \in \mathcal{H}$ gives $\Phi \in \mathcal{D}(A)$. This proves that $A$ and $N$ are selfadjoint.

(ii) For each $n \in \mathbb{N}$, the map $t \mapsto e^{i t A} e^{-i t A_{n}} \Psi^{(n)}$ is of class $C^{1}$ for any $\Psi^{(n)} \in$ $\mathcal{D}\left(A_{n}\right)$ by Stone's theorem with derivative

$$
\frac{d}{d t} e^{i t A} e^{-i t A_{n}} \Psi^{(n)}=i e^{i t A}\left(A-A_{n}\right) e^{-i t A_{n}} \Psi^{(n)}=0 .
$$

Hence, for any $\Psi \in \oplus_{n \in \mathbb{N}}^{\text {alg }} \mathcal{D}\left(A_{n}\right)$ (and then for any $\Psi \in \mathcal{H}$, since $\oplus_{n \in \mathbb{N}}^{\text {alg }} \mathcal{D}\left(A_{n}\right)$ is dense in $\mathcal{H}$ ) we see that for all $t \in \mathbb{R}$

$$
e^{i t A} \Psi=\sum_{n=0}^{\infty} e^{i t A_{n}} \Psi^{(n)}
$$

By functional calculus we extend identity (A.3) to any bounded Borel function $f$ on $\mathbb{R}$.

(iii) By using (ii), we get for all $s, t \in \mathbb{R}$ and $\Psi \in \mathcal{H}$

$$
e^{i t N} e^{i s A} \Psi=e^{i t N} \sum_{n=0}^{\infty} e^{i s A_{n}} \Psi^{(n)}=\sum_{n=0}^{\infty} e^{i t n} e^{i S A_{n}} \Psi^{(n)}=e^{i s A} e^{i t N} \Psi .
$$

(iv) The algebraic direct sum $\mathcal{D}_{\text {fin }}=\oplus_{n \in \mathbb{N}}^{\text {alg }} \mathcal{D}\left(A_{n}\right) \subset \mathcal{D}(A)$ is dense in $\mathcal{H}$ and invariant with respect to the group $\left(e^{i t A}\right)_{t \in \mathbb{R}}$. Therefore, $\mathcal{D}_{\text {fin }}$ is a core for $A$. On the other hand, the subspace $\mathcal{D}_{\text {fin }}^{0}=\oplus_{n \in \mathbb{N}}^{\text {alg }} \mathcal{D}_{n}$ satisfies

$$
A_{\mid \mathcal{D}_{\text {fin }}} \subset \overline{A_{\mid \mathcal{D}_{\text {fin }}^{0}}} \subset A_{\mid \mathcal{D}(A)} .
$$

Hence $\mathcal{D}_{\text {fin }}^{0}$ is also a core for $A$ since $\overline{A_{\mid \mathcal{D}_{\text {fin }}}}=A_{\mid \mathcal{D}(A)}$.

(v) The operator $B=\sum_{n=0}^{\infty} A_{n}+p(n) \mathbb{1}_{\mathcal{H}_{n}}$ (with its natural domain) is self-adjoint by assertion (i). It is clear that

$$
\mathcal{D}_{\text {fin }}=\oplus_{n \in \mathbb{N}}^{\text {alg }} \mathcal{D}\left(A_{n}\right) \subset \mathcal{D}(A) \cap \mathcal{D}(p(N)) \subset \mathcal{D}(B),
$$

and furthermore

$$
B_{\mid \mathcal{D}_{\text {fin }}}=A+p(N)_{\mid \mathcal{D}_{\text {fin }}} \subset A+p(N)_{\mid \mathcal{D}(A) \cap \mathcal{D}(p(N))} \subset B_{\mid \mathcal{D}(B)} .
$$

Therefore, the operator $A+p(N)_{\mid \mathcal{D}(A) \cap \mathcal{D}(p(N))}$ is essentially self-adjoint since $\overline{B_{\mid \mathcal{D}_{\text {fin }}}}=B_{\mid \mathcal{D}(B)}$. 


\section{B. Second quantization}

For the reader's convenience, the general framework of second quantization and some related notations are recalled. The phase-space, a complex separable Hilbert space, is denoted by $\mathcal{Z}$ with the scalar product $\langle.,$.$\rangle . The symmetric Fock space$ over $\mathcal{Z}$ is defined as the following direct Hilbert sum

$$
\Gamma_{s}(\mathcal{Z})=\bigoplus_{n=0}^{\infty} \bigvee^{n} \mathcal{Z}
$$

where $\bigvee^{n} \mathcal{Z}$ is the $n$-fold symmetric tensor product. The orthogonal projection from $\mathcal{Z}^{\otimes n}$ onto the closed subspace $\bigvee^{n} \mathcal{Z}$ is given by

$$
\mathcal{S}_{n}\left(\xi_{1} \otimes \xi_{2} \cdots \otimes \xi_{n}\right)=\frac{1}{n !} \sum_{\sigma \in \mathfrak{S}_{n}} \xi_{\sigma(1)} \otimes \xi_{\sigma(2)} \cdots \otimes \xi_{\sigma(n)} .
$$

Algebraic direct sums or tensor products are denoted with an alg superscript. Hence

$$
\mathcal{H}_{\text {fin }}=\bigoplus_{n \in \mathbb{N}}^{\text {alg }} \bigvee^{n} \mathcal{Z}
$$

denotes the subspace of vectors with a finite number of particles. The creation and annihilation operators $a^{*}(z)$ and $a(z)$, parameterized by $\varepsilon>0$, are then defined by:

$$
\begin{aligned}
a(z) \varphi^{\otimes n} & =\sqrt{\varepsilon n}\langle z, \varphi\rangle \varphi^{\otimes(n-1)} \\
a^{*}(z) \varphi^{\otimes n} & =\sqrt{\varepsilon(n+1)} \mathcal{S}_{n+1}\left(z \otimes \varphi^{\otimes n}\right), \quad \forall \varphi, z \in \mathcal{Z} .
\end{aligned}
$$

They extend to closed operators and they are adjoint of one another. They also satisfy the $\varepsilon$-canonical commutation relations (CCR):

$$
\left[a\left(z_{1}\right), a^{*}\left(z_{2}\right)\right]=\varepsilon\left\langle z_{1}, z_{2}\right\rangle \mathbb{1}, \quad\left[a^{*}\left(z_{1}\right), a^{*}\left(z_{2}\right)\right]=0=\left[a\left(z_{1}\right), a\left(z_{2}\right)\right] .
$$

The Weyl operators are given for $z \in \mathcal{Z}$ by

$$
W(z)=e^{\frac{i}{\sqrt{2}}\left[a^{*}(z)+a(z)\right]},
$$

and they satisfy Weyl commutation relations in the Fock space

$$
W\left(z_{1}\right) W\left(z_{2}\right)=e^{-\frac{i \varepsilon}{2} \operatorname{Im}\left\langle z_{1}, z_{2}\right\rangle} W\left(z_{1}+z_{2}\right), z_{1}, z_{2} \in \mathcal{Z} .
$$

The number operator is also parametrized by $\varepsilon>0$,

$$
\mathbf{N}_{\mid \bigvee^{n} \mathcal{Z}}=\varepsilon n \mathbb{1}_{\mid \bigvee^{n} \mathcal{Z}}
$$

For any self-adjoint operator $A: \mathcal{Z} \supset \mathcal{D}(A) \rightarrow \mathcal{Z}$, the operator $\mathrm{d} \Gamma(A)$ is the self-adjoint operator given by

$$
\mathrm{d} \Gamma(A)_{\mid \bigvee^{n, \text { alg }} \mathcal{D}(A)}=\varepsilon[\sum_{k=1}^{n} \mathbb{1} \otimes \cdots \otimes \underbrace{A}_{k} \otimes \cdots \otimes \mathbb{1}] .
$$




\section{B.1. Weyl, Anti-Wick quantized operators}

Let $\mathbb{P}$ denote the set of all finite-rank orthogonal projections on $\mathcal{Z}$ and for a given $\wp \in \mathbb{P}$ let $L_{\wp}(d z)$ denote the Lebesgue measure on the finite-dimensional subspace $\wp \mathcal{Z}$, with volume 1 for an orthonormal hypercube in $\wp \mathcal{Z}$. A function $f: \mathcal{Z} \rightarrow \mathbb{C}$ is said cylindrical if there exists $\wp \in \mathbb{P}$ and a function $g$ on $\wp \mathcal{Z}$ such that $f(z)=$ $g(\wp z)$, for all $z \in \mathcal{Z}$. In this case we say that $f$ is based on the subspace $\wp \mathcal{Z}$. We set $\mathcal{S}_{\text {cyl }}(\mathcal{Z})$ to be the cylindrical Schwartz space:

$$
\left(f \in \mathcal{S}_{\mathrm{cyl}}(\mathcal{Z})\right) \Leftrightarrow(\exists \wp \in \mathbb{P}, \exists g \in \mathcal{S}(\wp \mathcal{Z}), \quad f(z)=g(\wp z)) .
$$

The Fourier transform of a function $f \in \mathcal{S}_{\text {cyl }}(\mathcal{Z})$ based on the subspace $\wp \mathcal{Z}$ is defined as

$$
\mathcal{F}[f](\xi)=\int_{\wp \mathcal{Z}} f(z) e^{-2 \pi i \Re\langle z, \xi\rangle} L_{\wp}(d z)
$$

and its inverse Fourier transform as

$$
f(z)=\int_{\wp \mathcal{Z}} \mathcal{F}[f](\xi) e^{2 \pi i \Re\langle z, \xi\rangle} L_{\wp}(d \xi) .
$$

With any symbol $b \in \mathcal{S}_{\text {cyl }}(\mathcal{Z})$ based on $\wp \mathcal{Z}$, a Weyl observable can be associated according to

$$
b^{\text {Weyl }}=\int_{\wp \mathcal{Z}} \mathcal{F}[b](z) \quad W(\sqrt{2} \pi z) L_{\wp}(d z) .
$$

Notice that $b^{\text {Weyl }}$ is a well-defined bounded operator on $\mathcal{H}$ for all $b \in \mathcal{S}_{\text {cyl }}(\mathcal{Z})$ and that this quantization of cylindrical symbols depends on the parameter $\varepsilon$.

We also recall the Anti-Wick quantization through its usual finite-dimensional relation to Weyl operators:

$$
\begin{aligned}
b^{A-\text { Wick }} & =\left(b * \frac{e^{-\frac{|z|_{\wp \mathcal{Z}}^{2}}{\varepsilon / 2}}}{\wp \mathcal{Z}(\pi \varepsilon / 2)^{\operatorname{dim} \wp \mathcal{Z}}}\right)^{\text {Weyl }} \\
& =\int_{\wp \mathcal{Z}} \mathcal{F}[b](\xi) W(\sqrt{2} \pi \xi) e^{-\frac{\varepsilon \pi^{2}}{2}|\xi|_{\wp \mathcal{Z}}^{2}} L_{\wp}(d \xi),
\end{aligned}
$$

for any $b \in \mathcal{S}(\wp \mathcal{Z})$ by setting $b *_{\wp} \mathcal{Z} \gamma(z)=\int_{\wp} b(z) \gamma\left(z-z^{\prime}\right) L_{\wp}\left(d z^{\prime}\right)$.

\section{B.2. Wick quantized operators}

For any $p, q \in \mathbb{N}$, the space $\mathcal{P}_{p, q}(\mathcal{Z})$ of complex-valued polynomials on $\mathcal{Z}$ is defined with the following continuity condition: $b \in \mathcal{P}_{p, q}(\mathcal{Z})$ if and only if there exists $\tilde{b} \in \mathcal{L}\left(\bigvee^{p} \mathcal{Z}, \bigvee^{q} \mathcal{Z}\right)$ such that $b(z)=\left\langle z^{\otimes q}, \tilde{b} z^{\otimes p}\right\rangle$ 


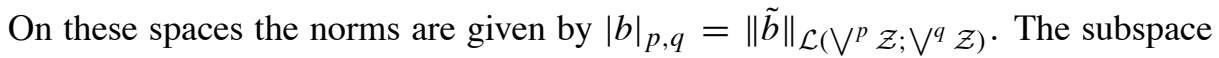
of $\mathcal{P}_{p, q}(\mathcal{Z})$ made of polynomials $b$ such that $\tilde{b}$ is a compact operator is denoted by $\mathcal{P}_{p, q}^{\infty}(\mathcal{Z})$. The Wick monomial of a 'symbol' $b \in \mathcal{P}_{p, q}(\mathcal{Z})$ is the linear operator

$$
b^{\text {Wick }}: \mathcal{H}_{\text {fin }} \rightarrow \mathcal{H}_{\text {fin }}
$$

defined as

$$
b_{\mid \bigvee^{\text {Wick }} \mathcal{Z}}=1_{[p,+\infty)}(n) \frac{\sqrt{n !(n+q-p) !}}{(n-p) !} \varepsilon^{\frac{p+q}{2}} \mathcal{S}_{n-p+q}\left(\tilde{b} \otimes \mathbb{1}^{(n-p)}\right),
$$

where $\tilde{b} \otimes \mathbb{1}^{(n-p)}$ is the operator with the action $\left(\tilde{b} \otimes \mathbb{1}^{(n-p)} \varphi^{\otimes n}=\left(\tilde{b} \varphi^{\otimes p}\right) \otimes\right.$ $\varphi^{\otimes(n-p)}$. Notice that $b^{\text {Wick }}$ depends on the scaling parameter $\varepsilon$. When $\tilde{b}$ is an unbounded operator with domain $\mathcal{D}(\tilde{b})$ containing $\bigvee^{p \text {,alg }} \mathcal{D}$, formula (B.6) makes sense when applied to $\Psi \in \bigvee^{n \text {,alg }} \mathcal{D}$.

Proposition B.1. For $b \in \mathcal{P}_{p, q}(\mathcal{Z})$ the following number estimate holds:

$$
\left|\langle\mathbf{N}\rangle^{-\frac{q}{2}} b^{\text {Wick }}\langle\mathbf{N}\rangle^{-\frac{p}{2}}\right|_{\mathcal{L}(\mathcal{H})} \leq|b|_{\mathcal{P}_{p, q}} .
$$

An important property of our class of Wick polynomials is that a composition of $b_{1}^{\text {Wick }} \circ b_{2}^{\text {Wick }}$ with $b_{1}, b_{2} \in \oplus_{p, q}^{\text {alg }} \mathcal{P}_{p, q}(\mathcal{Z})$ is a Wick polynomial with symbol in $\oplus_{p, q}^{\text {alg }} \mathcal{P}_{p, q}(\mathcal{Z})$. This was checked with a convenient writing in [4] and widely used also in $[5,6]$. We need some notation: For $b \in \mathcal{P}_{p, q}(\mathcal{Z})$, the $k$-th differential is well-defined according to

$$
\partial_{z}^{k} b(z) \in\left(\bigvee^{k} \mathcal{Z}\right)^{*} \quad \text { and } \quad \partial_{\bar{z}}^{k} b(z) \in \bigvee^{k} \mathcal{Z}
$$

for any fixed $z \in \mathcal{Z}$. Actually $\left(\bigvee^{k} \mathcal{Z}\right)^{*}$ is the dual of $\left(\bigvee^{k} \mathcal{Z}\right)$ with a $\mathbb{C}$-bilinear duality bracket. For two symbols $b_{i} \in \mathcal{P}_{p_{i}, q_{i}}(\mathcal{Z}), i=1,2$, and any $k \in \mathbb{N}$, the new symbol $\partial_{z}^{k} b_{1} \cdot \partial_{\bar{z}}^{k} b_{2}$ is now defined by

$$
\partial_{z}^{k} b_{1} \cdot \partial_{\bar{z}}^{k} b_{2}(z)=\left\langle\partial_{z}^{k} b_{1}(z), \partial_{\bar{z}}^{k} b_{2}(z)\right\rangle_{\left(\bigvee^{k} \mathcal{Z}\right)^{*}, \bigvee^{k} \mathcal{Z}}
$$

We also use the following notation for multiple Poisson brackets:

$$
\begin{aligned}
\left\{b_{1}, b_{2}\right\}^{(k)} & =\partial_{z}^{k} b_{1} \cdot \partial_{\bar{z}}^{k} b_{2}-\partial_{z}^{k} b_{2} \cdot \partial_{\bar{z}}^{k} b_{1}, \\
\left\{b_{1}, b_{2}\right\} & =\left\{b_{1}, b_{2}\right\}^{(1)} .
\end{aligned}
$$


With this notation the composition formula of Wick symbols has a very familiar form:

Proposition B.2. Let $b_{1} \in \mathcal{P}_{p_{1}, q_{1}}(\mathcal{Z})$ and $b_{2} \in \mathcal{P}_{p_{2}, q_{2}}(\mathcal{Z})$. For any $k \in$ $\left\{0, \ldots, \min \left\{p_{1}, q_{2}\right\}\right\}, \partial_{z}^{k} b_{1} . \partial_{\bar{z}}^{k} b_{2}$ belongs to $\mathcal{P}_{p_{1}+p_{2}-k, q_{1}+q_{2}-k}(\mathcal{Z})$ with the estimate

$$
\left|\partial_{z}^{k} b_{1} . \partial_{z}^{k} b_{2}\right|_{\mathcal{P}_{p_{1}+p_{2}-k, q_{1}+q_{2}-k}} \leq \frac{p_{1} !}{\left(p_{1}-k\right) !} \frac{q_{2} !}{\left(q_{2}-k\right) !}\left|b_{1}\right|_{\mathcal{P}_{p_{1}, q_{1}}}\left|b_{2}\right|_{\mathcal{P}_{p_{2}, q_{2}}} .
$$

The formulas

(i) $\begin{aligned} b_{1}^{\text {Wick }} \circ b_{2}^{\text {Wick }} & =\left(\sum_{k=0}^{\min \left\{p_{1}, q_{2}\right\}} \frac{\varepsilon^{k}}{k !} \partial_{z}^{k} b_{1} \cdot \partial_{\bar{z}}^{k} b_{2}\right)^{\text {Wick }} \\ & =\left(\left.e^{\varepsilon\left\langle\partial_{z}, \partial_{\bar{\omega}}\right\rangle} b_{1}(z) b_{2}(\omega)\right|_{z=\omega}\right)^{\text {Wick }},\end{aligned}$

(ii) $\left[b_{1}^{\text {Wick }}, b_{2}^{\text {Wick }}\right]=\left(\sum_{k=1}^{\max \left\{\min \left\{p_{1}, q_{2}\right\}, \min \left\{p_{2}, q_{1}\right\}\right\}} \frac{\varepsilon^{k}}{k !}\left\{b_{1}, b_{2}\right\}^{(k)}\right)^{\text {Wick }}$,

hold as identities on $\mathcal{H}_{\text {fin }}$.

Combined with Proposition B.1 and $\left(b^{\text {Wick }}\right)^{*}=(\overline{b(z)})^{\text {Wick }}$ this also gives:

Proposition B.3. For $b \in \mathcal{P}_{p, q}(\mathcal{Z}),\langle\mathbf{N}\rangle^{-\frac{(p+q)}{2}} b^{\text {Wick }}$ and $b^{\text {Wick }}\langle\mathbf{N}\rangle^{-\frac{(p+q)}{2}}$ extend as bounded operators on $\mathcal{H}$ with norm smaller that $C_{p, q}\|\tilde{b}\|_{\mathcal{L}\left(\bigvee^{p} \mathcal{Z} ; \bigvee^{q} \mathcal{Z}\right)}$ for all $\varepsilon \in(0, \bar{\varepsilon})$.

We will also need some more particular estimates stated in the following two results:

Lemma B.4. Let $A$ be a self-adjoint operator on $\mathcal{Z}$ with $A \geq \mathbb{1}$. For any polynomials $b_{1} \in \mathcal{P}_{1,2}(\mathcal{Z})$ and $b_{2} \in \mathcal{P}_{2,1}(\mathcal{Z})$ the following estimates hold true:

$$
\begin{aligned}
& \text { (i) }\left\|(\mathrm{d} \Gamma(A)+\sqrt{\mathbf{N}}+1)^{-1} b_{1}^{\text {Wick }}(\mathrm{d} \Gamma(A)+\sqrt{\mathbf{N}}+1)^{-1}\right\| \\
& \leq\left\|A^{-1 / 2} \tilde{b}_{1}\left(\mathbb{1} \otimes A^{-1 / 2}\right)\right\|_{\mathcal{L}\left(\bigvee^{2} \mathcal{Z}, \mathcal{Z}\right)} ; \\
& \text { (ii) }\left\|(\mathrm{d} \Gamma(A)+\sqrt{\mathbf{N}}+1)^{-1} b_{2}^{\text {Wick }}(\mathrm{d} \Gamma(A)+\sqrt{\mathbf{N}}+1)^{-1}\right\| \\
& \quad \leq\left\|\left(\mathbb{1} \otimes A^{-1 / 2}\right) \tilde{b}_{2} A^{-1 / 2}\right\|_{\mathcal{L}\left(\mathcal{Z}, \bigvee^{2} \mathcal{Z}\right)} .
\end{aligned}
$$

Remark B.5. The term $\sqrt{\mathbf{N}}$ can be absorbed in $\mathrm{d} \Gamma(A)+1$ if one accepts constants larger than 1 as factors of the right-hand sides of (i) and (ii). 
Proof. Estimate (ii) follows from (i) by taking the adjoint. Let us prove (i). For $\Phi, \Psi \in \oplus_{n \in \mathbb{N}}^{\text {alg }} \bigvee^{n, \text { alg }} \mathcal{D}(A)$, we write

$$
\begin{aligned}
& \left\langle\Psi, b_{1}^{\text {Wick }} \Phi\right\rangle \\
& =\sum_{n=2}^{\infty} \varepsilon^{3 / 2} \sqrt{n(n-1)^{2}}\left\langle\Psi^{(n-1)},\left(\tilde{b}_{1} \otimes \mathbb{1}^{(n-2)}\right) \Phi^{(n)}\right\rangle \\
& =\sum_{n=2}^{\infty} \varepsilon^{3 / 2} \sqrt{n(n-1)^{2}}\left\langle\left(A^{1 / 2} \otimes \mathbb{1}^{(n-2)}\right) \Psi^{(n-1)}, B_{A}\left(\mathbb{1} \otimes A^{1 / 2} \otimes \mathbb{1}^{(n-2)}\right) \Phi^{(n)}\right\rangle,
\end{aligned}
$$

with

$$
B_{A}=\left[\left(A^{-1 / 2} \tilde{b}_{1}\right)\left(\mathbb{1} \otimes A^{-1 / 2}\right)\right] \otimes \mathbb{1}^{(n-2)} .
$$

Hence, by the Cauchy-Schwarz inequality, we get

$$
\begin{aligned}
\left|\left\langle\Psi, b_{1}^{\text {Wick }} \Phi\right\rangle\right| \leq \| & A^{-1 / 2} \tilde{b}_{1}\left(\mathbb{1} \otimes A^{-1 / 2}\right) \|_{\mathcal{L}\left(\bigvee^{2} \mathcal{Z}, \mathcal{Z}\right)} \\
& \times\left(\sum_{n=2}^{\infty} \varepsilon^{3 / 2} \sqrt{n(n-1)^{2}}\left\|\left(A^{1 / 2} \otimes \mathbb{1}^{(n-2)}\right) \Psi^{(n-1)}\right\|^{2}\right)^{1 / 2} \\
& \times\left(\sum_{n=2}^{\infty} \varepsilon^{3 / 2} \sqrt{n(n-1)^{2}}\left\|\left(\mathbb{1} \otimes A^{1 / 2} \otimes \mathbb{1}^{(n-2)}\right) \Phi^{(n)}\right\|^{2}\right)^{1 / 2}
\end{aligned}
$$

Now, observe that

$$
\begin{aligned}
\varepsilon^{3 / 2} & \sqrt{n(n-1)^{2}}\left\|\left(A^{1 / 2} \otimes \mathbb{1}^{(n-2)}\right) \Psi^{(n-1)}\right\|^{2} \\
& \leq 2 \sqrt{\varepsilon(n-1)}\left\langle\Psi^{(n-1)},(\varepsilon(n-1))\left(A \otimes \mathbb{1}^{(n-2)}\right) \Psi^{(n-1)}\right\rangle \\
& \leq 2\left\langle\Psi^{(n-1)}, \sqrt{\mathbf{N}} \mathrm{d} \Gamma(A) \Psi^{(n-1)}\right\rangle
\end{aligned}
$$

and

$$
\begin{aligned}
\varepsilon^{3 / 2} \sqrt{n(n-1)^{2}}\left\|\left(\mathbb{1} \otimes A^{1 / 2} \otimes \mathbb{1}^{(n-2)}\right) \Phi^{(n)}\right\|^{2} & \leq \sqrt{n \varepsilon}\left\langle\Phi^{(n)}, n \varepsilon\left(\mathbb{1} \otimes A \otimes \mathbb{1}^{(n-2)}\right) \Phi^{(n)}\right\rangle \\
& \leq \sqrt{n \varepsilon}\left\langle\Phi^{(n)}, n \varepsilon\left(A \otimes \mathbb{1}^{(n-1)}\right) \Phi^{(n)}\right\rangle \\
& \leq\left\langle\Phi^{(n)}, \sqrt{\mathbf{N}} \mathrm{d} \Gamma(A) \Phi^{(n)}\right\rangle .
\end{aligned}
$$

On the other hand, with the inequality $2 a b \leq a^{2}+b^{2}$, we see that

$$
\begin{aligned}
& 2\left\langle\Psi^{(n-1)}, \sqrt{\mathbf{N}} \mathrm{d} \Gamma(A) \Psi^{(n-1)}\right\rangle \leq\left\langle\Psi^{(n-1)},\left(\mathbf{N}+\mathrm{d} \Gamma(A)^{2}\right) \Psi^{(n-1)}\right\rangle \\
& \leq\left\|(\sqrt{\mathbf{N}}+\mathrm{d} \Gamma(A)) \Psi^{(n-1)}\right\|^{2} \\
& \text { and } \quad 2\left\langle\Phi^{(n)}, \sqrt{\mathbf{N}} \mathrm{d} \Gamma(A) \Phi^{(n)}\right\rangle \leq\left\langle\Phi^{(n)},\left(\mathbf{N}+\mathrm{d} \Gamma(A)^{2}\right) \Psi^{(n)}\right\rangle \\
& \leq\left\|(\sqrt{\mathbf{N}}+\mathrm{d} \Gamma(A)) \Phi^{(n)}\right\|^{2},
\end{aligned}
$$


where the last inequalities come from $2 \sqrt{\mathbf{N}} \mathrm{d} \Gamma(A) \geq 0$. Therefore, we obtain $\left|\left\langle\Psi, b_{1}^{\text {Wick }} \Phi\right\rangle\right| \leq\left\|A^{-1 / 2} \tilde{b}_{1}\left(\mathbb{1} \otimes A^{-1 / 2}\right)\right\|\|(\sqrt{\mathbf{N}}+\mathrm{d} \Gamma(A)) \Psi\|\|(\sqrt{\mathbf{N}}+\mathrm{d} \Gamma(A)) \Phi\|$ and hence the estimate extends to $\Phi, \Psi \in \mathcal{D}(\sqrt{\mathbf{N}}+\mathrm{d} \Gamma(A)) \cap \mathcal{H}_{\text {fin }}$. This means that the operator $(\mathrm{d} \Gamma(A)+\sqrt{\mathbf{N}}+1)^{-1} b_{1}^{\text {Wick }}(\mathrm{d} \Gamma(A)+\sqrt{\mathbf{N}}+1)_{\mid \mathcal{D}(\sqrt{\mathbf{N}}+\mathrm{d} \Gamma(A)) \cap \mathcal{H}_{\text {fin }}}^{-1}$ extends to a bounded operator satisfying (i).

Lemma B.6. Let $A, B$ two self-adjoint operators on $\mathcal{Z}$ with $\mathcal{D}(A) \subset \mathcal{D}(B)$ and $B \geq 0$. Let $C$ be a self-adjoint operator on $\bigvee^{2} \mathcal{Z}$ such that $\mathcal{D}(C) \subset \mathcal{D}\left(B_{2}\right)$ where $B_{2}=B \otimes \mathbb{1}+\mathbb{1} \otimes B$. Then the following estimates hold true:

$$
\begin{gathered}
\left\|(\mathrm{d} \Gamma(B)+\mathbf{N}+1)^{-1} \mathrm{~d} \Gamma(A)(\mathrm{d} \Gamma(B)+\mathbf{N}+1)^{-1}\right\| \\
\quad \leq\left\|(1+B)^{-1} A(1+B)^{-1}\right\|_{\mathcal{L}(\mathcal{Z})} ; \\
\left\|(\mathrm{d} \Gamma(B)+\mathbf{N}+1)^{-1} C^{\mathrm{Wick}}(\mathrm{d} \Gamma(B)+\mathbf{N}+1)^{-1}\right\| \\
\quad \leq\left\|\left(1+B_{2}\right)^{-1 / 2} C\left(1+B_{2}\right)^{-1 / 2}\right\|_{\mathcal{L}\left(\bigvee^{2} \mathcal{Z}\right)} .
\end{gathered}
$$

Proof. We follow a similar argument as in the proof of Lemma B.4. Indeed, the Cauchy-Schwarz inequality gives for every $\Psi, \Phi \in \oplus_{n \in \mathbb{N}}^{\text {alg }} \bigvee^{n \text {,alg }} \mathcal{D}(B)$

$$
\begin{aligned}
|\langle\Psi, \mathrm{d} \Gamma(A) \Phi\rangle| \leq\left\|(1+B)^{-1} A(1+B)^{-1}\right\| \mathcal{L}(\mathcal{Z})\left(\sum_{n=1}^{\infty} \varepsilon n\left\|\left((1+B) \otimes \mathbb{1}^{(n-1)}\right) \Psi^{(n)}\right\|^{2}\right)^{1 / 2} \\
\times\left(\sum_{n=1}^{\infty} \varepsilon n\left\|\left((1+B) \otimes \mathbb{1}^{(n-1)}\right) \Phi^{(n)}\right\|^{2}\right)^{1 / 2} .
\end{aligned}
$$

Now, observe that

$$
\begin{aligned}
\varepsilon n\left\|\left((1+B) \otimes \mathbb{1}^{(n-1)}\right) \Psi^{(n)}\right\|^{2} & =\left\langle\Psi^{(n)}, \mathrm{d} \Gamma\left((1+B)^{2}\right) \Psi^{(n)}\right\rangle \\
& \leq\left\|\mathrm{d} \Gamma(1+B) \Psi^{(n)}\right\|_{\bigvee^{n} \mathcal{Z}}^{2},
\end{aligned}
$$

since in the sense of quadratic forms $\mathrm{d} \Gamma\left((1+B)^{2}\right) \leq \mathrm{d} \Gamma(1+B)^{2}$. Hence we obtain $|\langle\Psi, \mathrm{d} \Gamma(A) \Phi\rangle| \leq\left\|(1+B)^{-1} A(1+B)^{-1}\right\|_{\mathcal{L}(\mathcal{Z})}\|\mathrm{d} \Gamma(1+B) \Psi\|\|\mathrm{d} \Gamma(1+B) \Phi\|$.

This proves (i). Expressing $C^{\text {Wick }}$ as a quadratic form for $\Psi, \Phi \in \oplus_{n \in \mathbb{N}}^{\text {alg }} \bigvee^{n \text {,alg }} \mathcal{D}(B)$ and then applying the Cauchy-Schwarz inequality yields

$$
\begin{aligned}
\left|\left\langle\Psi, C^{\text {Wick }} \Phi\right\rangle\right| \leq & \left\|(\mathbb{1}+B \otimes \mathbb{1}+\mathbb{1} \otimes B)^{-1 / 2} C(\mathbb{1}+B \otimes \mathbb{1}+\mathbb{1} \otimes B)^{-1 / 2}\right\|_{\mathcal{L}\left(\bigvee^{2} \mathcal{Z}\right)} \\
& \times\left(\sum_{n=2}^{\infty} \varepsilon^{2} n(n-1)\left\|\left[(\mathbb{1}+B \otimes \mathbb{1}+\mathbb{1} \otimes B)^{1 / 2} \otimes \mathbb{1}^{(n-2)}\right] \Psi^{(n)}\right\|^{2}\right)^{1 / 2} \\
& \times\left(\sum_{n=2}^{\infty} \varepsilon^{2} n(n-1)\left\|\left[(\mathbb{1}+B \otimes \mathbb{1}+\mathbb{1} \otimes B)^{1 / 2} \otimes \mathbb{1}^{(n-2)}\right] \Phi^{(n)}\right\|^{2}\right)^{1 / 2} .
\end{aligned}
$$


Due to the symmetry of the vector $\Phi^{(n)}$ we remark that

$$
\begin{aligned}
\varepsilon^{2} n^{2} & \left\|\left[(\mathbb{1}+B \otimes \mathbb{1}+\mathbb{1} \otimes B)^{1 / 2} \otimes \mathbb{1}^{(n-2)}\right] \Phi^{(n)}\right\|^{2} \\
& =\varepsilon^{2} n^{2}\left\langle\Phi^{(n)},\left[(\mathbb{1}+B \otimes \mathbb{1}+\mathbb{1} \otimes B) \otimes \mathbb{1}^{(n-2)}\right] \Phi^{(n)}\right\rangle \\
& =\left\langle\Phi^{(n)},\left(\mathbf{N}^{2}+2 \mathrm{~d} \Gamma(B) \mathbf{N}\right) \Phi^{(n)}\right\rangle \\
& \leq\left\|(1+\mathrm{d} \Gamma(B)+\mathbf{N}) \Phi^{(n)}\right\|^{2} .
\end{aligned}
$$

So we obtain

$$
\begin{gathered}
\left|\left\langle\Psi, C^{\text {Wick }} \Phi\right\rangle\right| \leq\left\|\left(1+B_{2}\right)^{-1 / 2} C\left(1+B_{2}\right)^{-1 / 2}\right\|_{\mathcal{L}\left(\bigvee^{2} \mathcal{Z}\right)}\|(1+\mathrm{d} \Gamma(B)+\mathbf{N}) \Psi\| \\
\times\|(1+\mathrm{d} \Gamma(B)+\mathbf{N}) \Phi\| .
\end{gathered}
$$

This proves (ii).

\section{Absolutely continuous curves in $\operatorname{Prob}_{2}\left(\mathcal{Z}_{1, \mathbb{R}}\right)$}

This section firstly gathers results presented in [3] about Borel probability measures on a separable real Hilbert space which are weak solutions to continuity equations. In a second step, we shall adapt it to a complex Hilbert space $\mathcal{Z}_{1}$ endowed with its real euclidean structure.

\section{C.1. Absolutely continuous curves in $\operatorname{Prob}_{2}(E)$}

Let $E$ be a real Hilbert space, with scalar product $\langle$,$\rangle and norm ||$. The symbol $\operatorname{Prob}_{p}(E)$ (respectively $\left.\operatorname{Prob}(E)\right)$ refers to the set of Borel probability measures $\mu$ on $E$ such that $\int_{E}|x|^{p} d \mu(x)<+\infty$ (respectively with no momentum condition), and we simply work with $p=2$. On $\operatorname{Prob}_{2}(E)$, the 2-Wasserstein distance, $W_{2}$, is defined by

$$
W_{2}^{2}\left(\mu^{1}, \mu^{2}\right):=\min \left\{\int_{E^{2}}\left|x_{1}-x_{2}\right|_{E}^{2} d \mu\left(x_{1}, x_{2}\right) ; \Pi_{j, *} \mu=\mu_{j}\right\},
$$

where $\Pi_{j}: E^{2} \rightarrow E$ is the natural projection, $j=1,2$. The narrow convergence of a sequence $\left(\mu_{n}\right)_{n \in \mathbb{N}}$ of $\operatorname{Prob}_{2}(E)$, with a uniform control of $\int_{E}|x|^{2} d \mu_{n}$ is equivalent to the $W_{2}$ convergence on $\operatorname{Prob}_{2}(E)$ (see [3, Proposition 7.1.5]). Remember also that the tightness property of subsets of $\operatorname{Prob}_{2}(E)$ can be checked in the infinite-dimensional case with the weak topology, or after introducing a Hilbert basis $\left(e_{n}\right)_{n \in \mathbb{N}^{*}}$, with the distance $d_{\omega}\left(x_{1}, x_{2}\right)=\sqrt{\sum_{n \in \mathbb{N}^{*}} \frac{\left|\left\langle x_{1}-x_{2}, e_{n}\right\rangle\right|^{2}}{(1+n)^{2}}}$. This use of weak or $d_{\omega}$ topology is done also when considering probability measures on the set of absolutely continuous curves in $E$.

This tightness property is called the weak tightness property in [3] since it refers to the weak topology on $E$. Especially when one considers the narrow convergence in $\operatorname{Prob}_{2}(E)$, there is a weak narrow convergence and a strong narrow 
convergence (see the discussions about this in [3, Chapter 5 and 7]). The terms "narrow convergence" or "narrow continuity" refer to the strong ones and we shall specify "weak narrow convergence" and "weak narrow continuity" when necessary.

We recall two results of [3] and give a complete proof in the infinite-dimensional case of the second one, for the sake of completeness (it is left as an exercise to the reader in [3]).

The following result is the second part in [3, Theorem 8.3.1] with $p=2$. Although it is not clearly stated in [3, Theorem 8.3.1], the proof contains a "weak $\Rightarrow$ strong" result about the narrow continuity with respect to time.

Proposition C.1. Let I be an open interval in $\mathbb{R}$. If a weakly narrowly continuous curve $\mu_{t}: I \rightarrow \operatorname{Prob}_{2}(E)$ satisfies the continuity equation

$$
\partial_{t} \mu_{t}+\nabla^{T}\left(v_{t} \mu_{t}\right)=0
$$

in the weak sense

$$
\int_{I} \int_{E}\left(\partial_{t} \varphi(x, t)+\left\langle v_{t}(x), \nabla_{x} \varphi(x, t)\right\rangle_{E}\right) d \mu_{t}(x) d t=0, \forall \varphi \in \mathcal{C}_{0, \mathrm{cyl}}^{\infty}(E \times I),
$$

for some Borel velocity field $v_{t}$, with $\left|v_{t}\right|_{L^{2}\left(E, \mu_{t}\right)} \in L^{1}(I)$, then $\mu_{t}: I \rightarrow \operatorname{Prob}_{2}(E)$ is absolutely continuous with $W_{2}\left(\mu_{t^{\prime}}, \mu_{t}\right) \leq \int_{t}^{t^{\prime}}\left|v_{s}\right|_{L^{2}\left(E, \mu_{s}\right)} d s$. Moreover for Lebesgue-almost every $t \in I, v_{t}$ belongs to the closure in $L^{2}\left(E, \mu_{t}\right)$ of the subspace spanned by $\left\{\nabla \varphi, \varphi \in \mathcal{C}_{0, \mathrm{cyl}}^{\infty}(E)\right\}$.

Proof. The proof is given in [3]. We simply insist here on the "weak $\Rightarrow$ strong" narrow continuity argument. The proof of Theorem 8.3.1 ends with the following statements. For any time $t \in I, \mu_{t}$ is the weak narrow limit of a sequence $\left(\hat{\mu}_{t}^{d}\right)_{d \in \mathbb{N}}$ (of which the definition is recalled below) which satisfies

$$
W_{2}\left(\hat{\mu}_{t_{2}}^{d}, \hat{\mu}_{t_{1}}^{d}\right) \leq \int_{t_{1}}^{t_{2}}\left|v_{t}\right|_{L^{2}\left(E, \mu_{t}\right)} d t .
$$

Then the authors refer to the weak narrow lower semicontinuity of $W_{2}$,

$$
W_{2}\left(\mu_{t_{2}}, \mu_{t_{1}}\right) \leq \liminf _{d \rightarrow \infty} W_{2}\left(\hat{\mu}_{t_{2}}^{d}, \hat{\mu}_{t_{1}}^{d}\right)
$$

stated in their Lemma 7.1.4 and relation (7.1.11).

This implies absolute continuity in terms of $W_{2}$ and the narrow continuity with respect to time:

$$
W_{2}\left(\mu_{t_{1}}, \mu_{t_{2}}\right) \leq \int_{t_{1}}^{t_{2}}\left|v_{t}\right|_{L^{2}\left(E, \mu_{t}\right)} d t .
$$

Finally notice that the weak narrow continuity of $\mu_{t}$ suffices for the strong narrow continuity of $\hat{\mu}_{t}^{d}$ with respect to $t \in I$ because $\hat{\mu}_{t}^{d}$ is constructed after taking the image of $\mu_{t}$ via a finite-rank projection. 
The previous result concerns non-regular (non-Lipschitz) vector fields for which there is no uniqueness result for the Cauchy problem. Remember that the infinite-dimensional case, which relies on the cylindrical integration of $v_{t}$ and cylindrical disintegration of the measure $\mu_{t}$, requires the introduction of such singular vector fields (see the proof in [3, Theorem 8.3.1]). Nevertheless an interpretation of the continuity equation (C.1)(C.2) in terms of characteristic curves can be done via a probabilistic representation. For the sake of completeness, we adapt the proof stated in [3, Theorem 8.2.1] for the finite-dimensional case, to our infinite-dimensional case. For $T \in(0,+\infty)$, consider the set $\Gamma_{T}=\mathcal{C}^{0}([-T, T] ; E)$ endowed with the norm $|\gamma|_{\infty, T}=\max _{t \in[-T, T]}|\gamma(t)|_{E}$ or for weak topology argument with the distance $\max _{t \in[-T, T]} d_{\omega}\left(\gamma_{1}(t), \gamma_{2}(t)\right)$. For a Borel probability measure $\eta$ defined on $E \times \Gamma_{T}$, consider the time dependent Borel probability measure $\mu_{t}^{\eta}$ defined by

$$
\int_{E} \varphi d \mu_{t}^{\eta}=\int_{E \times \Gamma_{T}} \varphi(\gamma(t)) d \boldsymbol{\eta}(x, \gamma), \quad \forall \varphi \in \mathcal{C}_{b, \mathrm{cyl}}^{0}(E), t \in[-T, T] .
$$

The measure $\mu_{t}^{\eta}$ is the push-forward of $\boldsymbol{\eta}$ by the evaluation map

$$
e_{t}:(x, \gamma) \in E \times \Gamma_{T} \rightarrow \gamma(t) \in E, \quad \text { for } t \in[-T, T] .
$$

Proposition C.2. Let $\mu_{t}:[-T, T] \rightarrow \operatorname{Prob}_{2}(E)$ be a $W_{2}$-continuous solution to the continuity equation (C.1) and (C.2), with $I=(-T, T)$, for a suitable Borel vector field $v(t, x)=v_{t}(x)$ such that $\left|v_{t}\right|_{L^{2}\left(E, \mu_{t}\right)} \in L^{1}([-T, T])$. Then there exists a Borel probability measure $\eta$ in $E \times \Gamma_{T}$ such that:

(i) $\eta$ is concentrated on the set of pairs $(x, \gamma)$ such that $\gamma \in A C^{2}([-T, T] ; E)$ is a solution to the ODE $\dot{\gamma}(t)=v_{t}(\gamma(t))$ for Lebesgue almost every $t \in(-T, T)$ with $\gamma(0)=x$;

(ii) $\mu_{t}=\mu_{t}^{\eta}$ for any $t \in[-T, T]$, with $\mu_{t}^{\eta}$ defined as in (C.3).

Conversely, any $\boldsymbol{\eta}$ satisfying (i) and

$$
\int_{0}^{T} \int_{E \times \Gamma_{T}}\left|v_{t}(\gamma(t))\right|_{E} d \boldsymbol{\eta}(x, \gamma) d t<+\infty
$$

induces via (C.3) a solution to the continuity equation, with $\mu_{0}=\gamma(0)_{*} \eta$.

Remark C.3. The notation $A C^{2}([-T, T] ; E)$ refers to the set of absolutely continuous curves in $E$ with $L^{2}([-T, T] ; E)$ derivative. We keep the notation $\Phi_{*} \mu$ of differential geometry, for the push-forward or direct image of a measure $\mu$, by the Borel map $\Phi$.

Proof. The result is proved in [3] when $E$ is finite-dimensional. The proof of the second (converse) part of the statement is exactly the same as in finite dimension, after replacing regular (Lipschitz) test functions by cylindrical ones. We now show, for the first part, how the infinite-dimensional case is deduced from 
the finite-dimensional result, following an approximation scheme like in the proof of [3, Theorem 8.3.1]. After introducing an Hilbert basis $\left(e_{n}\right)_{n \in \mathbb{N}^{*}}$ of $E$, the maps $\pi^{d}: E \rightarrow \mathbb{R}^{d}, \pi^{d, T}: \mathbb{R}^{d} \rightarrow E$ and $\hat{\pi}^{d}: E \rightarrow E$ are defined according to

$$
\begin{aligned}
\pi^{d}(x) & =\left(\left\langle e_{1}, x\right\rangle, \ldots,\left\langle e_{d}, x\right\rangle\right), \\
\pi^{d, T}\left(y_{1}, \ldots, y_{d}\right) & =\sum_{j=1}^{d} y_{j} e_{j}, \\
\hat{\pi}^{d} & =\pi^{d, T} \circ \pi^{d} .
\end{aligned}
$$

With the measure $\mu_{t} \in \operatorname{Prob}_{2}(E)$, the measure $\mu_{t}^{d} \in \operatorname{Prob}_{2}\left(\mathbb{R}^{d}\right)$ is defined by $\mu_{t}^{d}=\pi_{*}^{d} \mu_{t}$ and $\left\{\mu_{t, y}, y \in \mathbb{R}^{d}\right\}$ denotes the disintegration of $\mu_{t}$ with respect to $\mu_{t}^{d}$. Within the space $E$ endowed with the basis $\left(e_{n}\right)_{n \in \mathbb{N}^{*}}, \hat{\mu}_{t}^{d}$ is nothing but $\mu_{t}^{d} \otimes \delta_{0}$ in the decomposition $Z=F_{d} \times F_{d}^{\perp}$ with $F_{d}=\operatorname{span}\left(e_{1}, \ldots, e_{d}\right)$. The vector field $v_{t}^{d}$ (respectively $\hat{v}_{t}^{d}$ ) is defined on $\mathbb{R}^{d}$ (respectively on $E$ ) by

$$
\begin{aligned}
v_{t}^{d}(y) & =\int_{\left(\pi^{d}\right)^{-1}(y)} \pi^{d} v_{t}(x) d \mu_{t, y}(x), \quad y \in \mathbb{R}^{d} \\
\text { respectively } \hat{v}_{t}^{d}(y) & =\int_{\left(\hat{\pi}^{d}\right)^{-1}\left(\hat{\pi}^{d} y\right)} \hat{\pi}^{d} v_{t}(x) d \mu_{t, \pi^{d} y}(x), \quad y \in E .
\end{aligned}
$$

Within the proof of Theorem 8.3.1 in [3], it was checked that $\mu_{t}^{d}$ (respectively $\hat{\mu}_{t}^{d}$ ) is a weak solution to the continuity equation

$$
\begin{array}{ll} 
& \partial_{t} \mu_{t}^{d}+\nabla^{T}\left(v_{t}^{d} \mu_{t}^{d}\right)=0, \\
\text { respectively } & \partial_{t} \hat{\mu}_{t}^{d}+\nabla^{T}\left(\hat{v}_{t}^{d} \hat{\mu}_{t}^{d}\right)=0
\end{array}
$$

with the following properties:

1) $\left|\hat{v}_{t}^{d}\right|_{L^{2}\left(E, \hat{\mu}_{t}^{d}\right)}=\left|v_{t}^{d}\right|_{L^{2}\left(\mathbb{R}^{d}, \mu_{t}^{d}\right)} \leq\left|v_{t}\right|_{L^{2}\left(E, d \mu_{t}\right)}$;

2) $W_{2}\left(\mu_{t_{1}}^{d}, \mu_{t_{2}}^{d}\right) \leq \int_{t_{1}}^{t_{2}}\left|v_{t}^{d}\right|_{L^{2}\left(\mathbb{R}^{d}, \mu_{t}^{d}\right)} d t \leq \int_{t_{1}}^{t_{2}}\left|v_{t}\right|_{L^{2}\left(E, \mu_{t}\right)} d t$, for $-T<t_{1} \leq t_{2}<$ $T$

3) the sequence $\left(\hat{\mu}_{t}^{d}\right)_{d \in \mathbb{N}^{*}}$ converges weakly narrowly to $\mu_{t}$ with the estimate

$$
W_{2}\left(\mu_{t_{1}}, \mu_{t_{2}}\right) \leq \liminf _{d \rightarrow \infty} W_{2}\left(\mu_{t_{1}}^{d}, \mu_{t_{2}}^{d}\right) \leq \int_{t_{1}}^{t_{2}}\left|v_{t}\right|_{L^{2}\left(E, \mu_{t}\right)} d t,-T<t_{1} \leq t_{2}<T .
$$

Additionally a time rescaling argument (see in [3, Lemma 1.1.4 and Theorem 8.1.3]) allows to assume without restriction

$$
\left|v_{t}\right|_{L^{2}\left(E, \mu_{t}\right)} \in L^{\infty}((-T, T)) .
$$

The set of continuous maps from $[-T, T]$ to $\mathbb{R}^{d}$ is denoted by $\Gamma_{T}^{d}$. The mapping from $\Gamma_{T}$ to $\Gamma_{T}^{d}$, still denoted by $\pi^{d}$ is defined by $\left[\pi^{d} \gamma\right](t)=\pi^{d}(\gamma(t))$. By using 
the finite-dimensional result, stated in Theorem 8.2.1 of [3], there exists for any $d \in \mathbb{N}$ a probability measure, $\boldsymbol{\eta}^{d}$, on $\mathbb{R}^{d} \times \Gamma_{T}^{d}$ such that the properties (i) and (ii) hold when $\left(\mu_{t}, v_{t}, E\right)$ is replaced by $\left(\mu_{t}^{d}, v_{t}^{d}, \mathbb{R}^{d}\right)$. Equivalently the result can be formulated in $E$ after using $\left(\hat{\mu}_{t}^{d}, \hat{v}_{t}^{d}, E\right)$ instead of $\left(\mu_{t}^{d}, v_{t}^{d}, \mathbb{R}^{d}\right)$ and using $\hat{\eta}^{d}=$ $\left(\pi^{d, T} \times \pi^{d, T}\right)_{*} \eta^{d}$. Hence we have a sequence $\left(\hat{\eta}^{d}\right)_{d \in \mathbb{N}}$ of probability measures on $E \times \Gamma_{T}$ which satisfy

$$
\begin{aligned}
\int_{E} \varphi \circ \pi^{d} d \hat{\mu}_{t}^{d} & =\int_{\mathbb{R}^{d}} \varphi d \mu_{t}^{d}=\int_{\mathbb{R}^{d} \times \Gamma_{T}^{d}} \varphi(\gamma(t)) d \boldsymbol{\eta}^{d}(x, \gamma) \\
& =\int_{E \times \Gamma_{T}} \varphi \circ \pi^{d}(\gamma(t)) d \hat{\boldsymbol{\eta}}^{d}(x, \gamma), \forall \varphi \in \mathcal{C}_{b}^{0}\left(\mathbb{R}^{d}\right), t \in[-T, T],
\end{aligned}
$$

where $\varphi \circ \pi^{d}$ can be replaced by $\varphi \circ \hat{\pi}^{d}$ with $\varphi \in \mathcal{C}_{b}^{0}\left(F_{d}\right)$.

After some regularization done in [3, pages 179-180], it is proved that any measure $\hat{\boldsymbol{\eta}}^{d}$ satisfies

$$
\int_{\Gamma_{T}} \int_{-T}^{T}|\dot{\gamma}(t)|^{2} d t d \hat{\boldsymbol{\eta}}^{d} \leq \int_{-T}^{T} \int_{E}\left|v_{t}^{d}(x)\right|^{2} d \hat{\mu}_{t}^{d} d t \leq \int_{-T}^{T} \int_{E}\left|v_{t}(x)\right|^{2} d \hat{\mu}_{t} d t .
$$

Since the functional $g \rightarrow \int_{-T}^{T}|\dot{g}(t)|^{2} d t$, defined on $\left\{g \in \Gamma_{T}: g(0)=0\right\}$ and set to $+\infty$ if $g \notin A C^{2}([-T, T] ; E)$, has compact sublevel sets in $\Gamma_{T}$, the two mappings

$$
r^{1}:(x, \gamma) \in E \times \Gamma_{T} \rightarrow x \in E, r^{2}:(x, \gamma) \in E \times \Gamma_{T} \rightarrow g_{\gamma, x}=\gamma-x \in \Gamma_{T}
$$

give rise to (weakly) tight families of marginals $\left(r_{*}^{1} \hat{\boldsymbol{\eta}}^{d}\right)_{d \in \mathbb{N}}=\left(\hat{\mu}_{0}^{d}\right)_{d \in \mathbb{N}}$ and $\left(r_{*}^{2} \hat{\boldsymbol{\eta}}^{d}\right)_{d \in \mathbb{N}}$. Remember that the compactness of subsets of $E$ or $\Gamma_{T}$ is considered with the weak topology on $E$ or the distance $d_{\omega}$. Hence the family $\left(\hat{\eta}^{d}\right)_{d \in \mathbb{N}}$ is (weakly) tight in $\operatorname{Prob}\left(E \times \Gamma_{T}\right)$ and we take for $\boldsymbol{\eta}$ a weak narrow limit point of $\hat{\boldsymbol{\eta}}^{d}$. By assuming the test function $\varphi$ in (C.5) to depend only on $d^{\prime}$ coordinates with $d^{\prime} \leq d$, and by taking the limit $d \rightarrow+\infty$ while $d^{\prime}$ and $\varphi$ are fixed, we get

$$
\int_{E}\left(\varphi \circ \pi^{d^{\prime}}\right) d \mu_{t}=\int_{E \times \Gamma}\left(\varphi \circ \pi^{d^{\prime}}\right)(\gamma(t)) d \boldsymbol{\eta}(x, \gamma)
$$

for all $\varphi \in \mathcal{C}_{b}^{0}\left(\mathbb{R}^{d^{\prime}}\right)$ and $t \in[-T, T]$, where $\varphi \circ \pi^{d^{\prime}}$ can then be replaced by any cylindrical function or Borel bounded function on $E$. It remains to prove condition (i) for $\boldsymbol{\eta}$, namely that this measure is concentrated on curves verifying $\dot{\gamma}=v(\gamma(t))$ for Lebesgue almost every $t \in(-T, T)(\gamma(0)=x$ is already known).

The estimate (8.2.6) used in [3] for the finite-dimensional case, provides the inequality

$$
\int_{\mathbb{R}^{d} \times \Gamma_{T}^{d}}\left|\gamma(t)-x-\int_{0}^{t} w_{s}(\gamma(s)) d s\right|^{2} d \eta^{d}(x, \gamma) \leq(2 T) \int_{-T}^{T} \int_{\mathbb{R}^{d}}\left|v_{t}^{d}-w_{t}\right|^{2} d \mu_{t}^{d} d t,
$$


for any family $w_{s}(x)=w(s, x)$ of uniformly bounded continuous functions from $[-T, T] \times \mathbb{R}^{d}$ to $\mathbb{R}^{d}$. After assuming that $w$ actually belongs to $\mathcal{C}_{b}^{0}([-T, T] \times$ $\left.\mathbb{R}^{d^{\prime}} ; \mathbb{R}^{d^{\prime}}\right)$ with fixed $d^{\prime} \leq d$, using $\hat{w}_{t}=\pi^{d^{\prime}, T} \circ w_{t} \circ \pi_{d^{\prime}} \in \mathcal{C}_{b}^{0}([-T, T] \times E ; E)$, and taking the limit as $d \rightarrow \infty$ gives

$$
\int_{E \times \Gamma_{T}}\left|\gamma(t)-x-\int_{0}^{t} \hat{w}_{s}(\gamma(s)) d s\right|^{2} d \boldsymbol{\eta}(x, \gamma) \leq(2 T) \limsup _{d \rightarrow \infty} \int_{-T}^{T} \int_{\mathbb{R}^{d}}\left|\hat{v}_{t}^{d}-\hat{w}_{t}\right|^{2} d \hat{\mu}_{t}^{d} d t .
$$

But condition 1) for $\left|\hat{v}_{t}^{d}\right|_{L^{2}\left(E, \hat{\mu}_{t}^{d}\right)}$ is easily extended to

$$
\left|\hat{v}_{t}^{d}-\hat{w}_{t}\right|_{L^{2}\left(E, \hat{\mu}_{t}^{d}\right)} \leq\left|v_{t}-\hat{w}_{t}\right|_{L^{2}\left(E, \mu_{t}\right)}
$$

by the same argument, relying on

$$
\begin{aligned}
\left|\int_{E}\left\langle\hat{v}_{t}^{d}-\hat{w}_{t}, \chi\right\rangle d \hat{\mu}_{t}^{d}\right| & \leq\left|\int_{E}\left\langle\hat{\pi}_{d}\left(v_{t}(x)-\hat{w}_{t}(x)\right), \chi\left(\hat{\pi}^{d}(x)\right)\right\rangle d \mu_{t}(x)\right| \\
& \leq\left|v_{t}-\hat{w}_{t}\right|_{L^{2}\left(E ; \mu_{t}\right)}|\chi|_{L^{2}\left(E, \hat{\mu}_{t}^{d}\right)}, \quad \forall \chi \in L^{2}\left(E, \hat{\mu}_{t}^{d}\right) .
\end{aligned}
$$

This uniform upper bound leads to

$$
\int_{E \times \Gamma_{T}}\left|\gamma(t)-x-\int_{0}^{t} \hat{w}_{s}(\gamma(s)) d s\right|^{2} d \boldsymbol{\eta}(x, \gamma) \leq(2 T) \int_{0}^{T} \int_{E}\left|v_{t}(x)-\hat{w}_{t}(x)\right|^{2} d \mu_{t} d t .
$$

According to the last statement of Proposition C.1, $v_{t}$ can be approximated in $L^{2}\left(E, \mu_{t}\right)$ by a sequence of bounded regular cylindrical functions, $\left(\hat{w}_{t, n}\right)_{n \in \mathbb{N}}$. By possibly truncating with respect to times $t \rightarrow \hat{w}_{t, n}$ so that $\left|v_{t}-\hat{w}_{t, n}\right|_{L^{2}\left(E, \mu_{t}\right)} \leq 1$ for a.e. $t \in(-T, T)$ and all $n \in \mathbb{N}^{*}$, Lebesgue's Theorem implies

$$
\int_{E \times \Gamma_{T}}\left|\gamma(t)-x-\int_{0}^{t} v_{s}(\gamma(s)) d s\right|^{2} d \hat{\eta}(x, \gamma)=0
$$

which ends the proof.

Below is a consequence of the above probabilistic interpretation when the Cauchy problem $\dot{\gamma}(t)=v_{t}(\gamma(t)), \gamma(0)=x$ admits a unique solution for all $x \in E$. The fact that we have to pass by the probabilistic representation is a real question. Contrary to the finite-dimensional case, the well-posedness of the Cauchy problem, even with the standard Picard's contraction argument, defining a flow on the whole space $E$, does not give a representation formula for observables. The point is that the natural observables, or test functions, are cylindrical functions, a property which is not generally preserved by the nonlinear flow.

Proposition C.4. Let $\mu_{t}: \mathbb{R} \rightarrow \operatorname{Prob}_{2}(E)$ be a $W_{2}$-continuous solution to the continuity equation (C.1) and (C.2) for a suitable Borel velocity field $v(t, x)=$ 
$v_{t}(x)$ such that $\left|v_{t}\right|_{L^{2}\left(E, \mu_{t}\right)} \in L^{1}([-T, T])$ for all $T>0$. Assume additionally that the Cauchy problem

$$
\dot{\gamma}(t)=v_{t}(\gamma(t)), \quad \gamma(s)=x \quad \text { or } \quad \gamma(t)=x+\int_{s}^{t} v_{s}(\gamma(s)) d s,
$$

admits a unique global continuous solution on $\mathbb{R}$ for all $s \in \mathbb{R}$ and all $x \in E$, such that $\gamma(t)=\Phi(t, s) \gamma(s)$ defines a Borel flow on $E($ i.e., $\Phi(t, s): E \rightarrow E$ is a Borel function for all $t, s \in \mathbb{R})$. Then the measure $\mu_{t}$ satisfies

$$
\forall t, s \in \mathbb{R}, \quad \mu_{t}=\Phi(t, s)_{*} \mu_{s} .
$$

Proof. It suffices to work with $t \in[-T, T]$ as in Proposition C.2. Since the evaluation map $e_{t}:(x, \gamma) \in E \times \Gamma_{T} \rightarrow \gamma(t) \in E$ is a continuous, thus Borel, map. The relation $\mu_{t}=\mu_{t}^{\eta}$ defined according to (C.3) extends to any bounded Borel function $\varphi$ on $E$ :

$$
\int_{E} \varphi d \mu_{t}=\int_{E \times \Gamma_{T}} \varphi(\gamma(t)) d \eta(x, \gamma)
$$

Using $\gamma(t)=\Phi(t, s) \gamma(s)$, with $\Phi(t, s)$ Borel, we deduce

$$
\int_{E} \varphi d \mu_{t}=\int_{E \times \Gamma_{T}}[\varphi \circ \Phi(t, s)](\gamma(s)) d \eta(x, \gamma)=\int_{E}[\varphi \circ \Phi(t, s)] d \mu_{s}
$$

which is nothing but $\mu_{t}=\Phi(t, s)_{*} \mu_{s}$.

\section{C.2. Application to Hamiltonian fields}

We finally specify how these results apply to our case, when the phase-space $\mathcal{Z}_{0}$ is a complex Hilbert space and the velocity field is associated with a (singular) Hamiltonian vector field, only defined on $\mathcal{Z}_{1} \subset \mathcal{Z}_{0}$.

Consider a complex Hilbert triple $\mathcal{Z}_{1} \subset \mathcal{Z}_{0} \subset \mathcal{Z}_{-1}$, with $\mathcal{Z}_{1}$ densely continuously embedded in $\mathcal{Z}_{0}$ and $\mathcal{Z}_{-1}$ being the dual of $\mathcal{Z}_{1}$ for the duality bracket extending $\left\langle z_{1}, z_{2}\right\rangle \mathcal{Z}_{0}$. The dual of a complex Hilbert space $\mathcal{Z}$ while keeping the $\mathbb{C}$-bilinear duality bracket, written $u . v$ in (B.8), is still denoted by $\mathcal{Z}^{*}$. In the case treated in the article $\mathcal{Z}_{0}=L^{2}\left(\mathbb{R}^{d}, d x\right), \mathcal{Z}_{1}=H^{1}\left(\mathbb{R}^{d}\right)$ and $\mathcal{Z}_{-1}=H^{-1}\left(\mathbb{R}^{d}\right)$. The space $\mathcal{Z}_{0}$ is endowed with its scalar product $\left\langle z_{1}, z_{2}\right\rangle \mathcal{Z}_{0}$, real euclidean structure with $\mathfrak{R}\left\langle z_{1}, z_{2}\right\rangle \mathcal{Z}_{0}$ and its symplectic structure $\sigma\left(z_{1}, z_{2}\right)=\operatorname{Im}\left\langle z_{1}, z_{2}\right\rangle \mathcal{Z}_{0}$. On $\mathcal{Z}_{1}$ we will use the hermitian $\left\langle z_{1}, z_{2}\right\rangle \mathcal{Z}_{1}$ and euclidean scalar product

$$
\left\langle z_{1}, z_{2}\right\rangle_{\mathcal{Z}_{1}, \mathbb{R}}=\Re\left\langle z_{1}, z_{2}\right\rangle_{\mathcal{Z}_{1}} .
$$

For a cylindrical function $f \in \mathcal{S}_{\text {cyl }}\left(\mathcal{Z}_{0}\right)$, based on $\wp \mathcal{Z}_{0}$, the differentials $\partial_{z} f(z)$ and $\partial_{\bar{z}} f(z)$ are defined by

$$
\begin{aligned}
& \partial_{z} f(z)=\int_{\wp \mathcal{Z}_{0}} i \pi\langle\xi| e^{2 i \pi \Re\langle z, \xi\rangle} \mathcal{F}[f](\xi) L_{\wp}(d \xi) \\
& \partial_{\bar{z}} f(z)=\int_{\wp \mathcal{Z}_{0}} i \pi|\xi\rangle e^{2 i \pi \Re\langle z, \xi\rangle} \mathcal{F}[f](\xi) L_{\wp}(d \xi) .
\end{aligned}
$$


Hence $\partial_{z} f(z)$ is a continuous $\mathbb{C}$-linear form on $\mathcal{Z}_{0}$, while $\partial_{\bar{z}} f(z) \in \mathcal{Z}_{0}$. This notation is coherent with the definition of $\partial_{z} b(z)$ and $\partial_{\bar{z}} b(z)$ when $b$ is a Wick symbol in $\oplus_{p, q}^{\text {alg }} \mathcal{P}_{p, q}(\mathcal{Z})$. A function $f \in \mathcal{S}_{\text {cyl }}\left(\mathcal{Z}_{1}\right)$ is given by

$$
f(z)=\varphi\left(\left\langle\xi_{1}, z\right\rangle_{\mathcal{Z}_{1}}, \ldots,\left\langle\xi_{N}, z\right\rangle \mathcal{Z}_{1}\right)=\varphi\left(\left\langle\eta_{1}, z\right\rangle, \ldots,\left\langle\eta_{N}, z\right\rangle\right)
$$

with $\varphi\left(w_{1}, \ldots w_{N}\right) \in \mathcal{S}\left(\mathbb{R}^{N}\right)$ and $\xi_{1}, \ldots, \xi_{N} \in \mathcal{Z}_{1}$ and $\eta_{1}, \ldots, \eta_{N} \in \mathcal{Z}_{-1}$, such that $\left\langle\xi_{j}, z\right\rangle_{\mathcal{Z}_{1}}=\left\langle\eta_{j}, z\right\rangle$ for all $z \in \mathcal{Z}_{1}$. The derivatives $\partial_{z} f$ and $\partial_{\bar{z}} f$ are thus given by

$$
\begin{aligned}
& \forall z \in \mathcal{Z}_{1}, \quad \partial_{z} f(z)=\sum_{j=1}^{N} \partial_{w_{j}} \varphi\left(\left\langle\eta_{1}, z\right\rangle, \ldots,\left\langle\eta_{N}, z\right\rangle\right)\left\langle\eta_{j}\right| \quad \in \mathcal{Z}_{1}^{*}, \\
& \forall z \in \mathcal{Z}_{1}, \quad \partial_{\bar{z}} f(z)=\sum_{j=1}^{N} \partial_{\overline{w_{j}}} \varphi\left(\left\langle\eta_{1}, z\right\rangle, \ldots,\left\langle\eta_{N}, z\right\rangle\right)\left|\eta_{j}\right\rangle \quad \in \mathcal{Z}_{-1} .
\end{aligned}
$$

When $h(z)$ is an unbounded polynomial on $\mathcal{Z}_{0}$ but which happens to be a realvalued, Fréchet $\mathcal{C}^{1}$-function on $\mathcal{Z}_{1}$, the derivatives $\partial_{z} h(z)$ and $\partial_{\bar{z}} h(z)$ are defined only for $z \in \mathcal{Z}_{1}$ and we have

$$
\forall z \in \mathcal{Z}_{1}, \quad \partial_{\bar{z}} h(z) \in \mathcal{Z}_{1} \quad, \quad \partial_{z} h(z) \in \mathcal{Z}_{-1}^{*} .
$$

When $f \in \mathcal{S}_{\text {cyl }}\left(\mathcal{Z}_{0}\right)$ (respectively $g \in \mathcal{S}_{\text {cyl }}\left(\mathcal{Z}_{1}\right)$ or $h$ ) is real-valued, differentiating $f(z+t e)$ at $t=0, t \in \mathbb{R}$, for any $e \in \mathcal{Z}_{0}$ (respectively any $e \in \mathcal{Z}_{1}$ ) leads to

$$
\begin{array}{ll}
\overline{\partial_{z} f(z) \cdot u}=\left\langle u, \partial_{\bar{z}} f(z)\right\rangle, & z \in \mathcal{Z}_{0}, u \in \mathcal{Z}_{0} \\
\overline{\partial_{z} g(z) \cdot u}=\left\langle u, \partial_{\bar{z}} g(z)\right\rangle, & z \in \mathcal{Z}_{1}, u \in \mathcal{Z}_{1} \\
\overline{\partial_{z} h(z) \cdot u}=\left\langle u, \partial_{\bar{z}} h(z)\right\rangle, & z \in \mathcal{Z}_{1}, u \in \mathcal{Z}_{-1} .
\end{array}
$$

Note that the Poisson bracket

$$
i\{h, b\}(z)=i\left(\partial_{z} h . \partial_{\bar{z}} b-\partial_{z} b . \partial_{\bar{z}} h\right)(z), \quad z \in \mathcal{Z}_{1}
$$

is well-defined for $b \in \mathcal{S}_{\text {cyl }}\left(\mathcal{Z}_{1} ; \mathbb{R}\right)$, and our aim is to write it as the real scalar product

$$
\langle v(z),(\nabla b)(z)\rangle_{\mathcal{Z}_{1}, \mathbb{R}}, \quad z \in \mathcal{Z}_{1} .
$$

Definition C.5. For a cylindrical function on $\mathcal{Z}_{1}, f \in \mathcal{S}_{\text {cyl }}\left(\mathcal{Z}_{1}\right)$, the gradients $\nabla_{\bar{z}}$ and $\nabla$ are defined by

$$
\begin{aligned}
& \forall z \in \mathcal{Z}_{1}, u \in \mathcal{Z}_{1}, \quad\left\langle u, \nabla_{\bar{z}} f(z)\right\rangle_{\mathcal{Z}_{1}}=\left\langle u, \partial_{\bar{z}} f(z)\right\rangle, \\
& \nabla=2 \nabla_{\bar{z}} .
\end{aligned}
$$




\section{Remark C.6.}

- Although it is not necessary, these definitions can be justified by introducing a complex conjugation $u \rightarrow \bar{u}$ on $\mathcal{Z}_{0}$, which remains a conjugation on $\mathcal{Z}_{1}$, that is an isometric $\mathbb{C}$-antilinear application such that $\overline{\langle u, v\rangle_{\mathcal{Z}_{0,1}}}=\langle\bar{u}, \bar{v}\rangle_{\mathcal{Z}_{0,1}}$. When $\mathcal{Z}_{0}=L^{2}\left(\mathbb{R}^{d} ; \mathbb{C}\right)$ and $\mathcal{Z}_{1}=H^{1}\left(\mathbb{R}^{d}\right)$ this is the usual pointwise complex conjugation. For real valued functions, set

$$
\nabla_{R} f=\nabla_{\bar{z}} f+\nabla_{z} f \quad \text { and } \quad \nabla_{I} f=\frac{1}{i}\left(\nabla_{\bar{z}} f-\nabla_{z} f\right) .
$$

so that

$$
\nabla_{\bar{z}} f=\frac{1}{2}\left(\nabla_{R} f+i \nabla_{I} f\right) .
$$

Similarly, an element $X$ of $\mathcal{Z}_{1}$ can be written $X=X_{R}+i X_{I}$ with $\overline{X_{R, I}}=X_{R, I}$ or $X=\left(\begin{array}{c}X_{R} \\ X_{I}\end{array}\right)$ and the real scalar product

$$
\langle X, Y\rangle_{\mathcal{Z}_{1}, \mathbb{R}}=\Re\langle X, Y\rangle_{\mathcal{Z}_{1}}=\left\langle X_{R}, Y_{R}\right\rangle_{\mathcal{Z}_{1}}+\left\langle X_{I}, Y_{I}\right\rangle_{\mathcal{Z}_{1}} .
$$

Then the definition of the gradient of a real cylindrical function $f$ becomes

$$
\nabla f=\left(\begin{array}{c}
\nabla_{R} f \\
\nabla_{I} f
\end{array}\right)
$$

- It is important to notice that we do not use the $\mathcal{Z}_{1}$-gradient for the real-valued function $h(z)$, but keep the derivative, $\partial_{\bar{z}} h(z)$ modeled on the duality bracket $\langle$,$\rangle . With a complex conjugation and since h$ is real valued, it can be decomposed into $\partial_{\bar{z}} h=\frac{1}{2}\left(\partial_{R} h+i \partial_{I} h\right)$ and

$$
-i \partial_{\bar{z}} h=\frac{1}{2} \partial_{I} h-\frac{i}{2} \partial_{R} h .
$$

Lemma C.7. With the above notation and assumptions the equality

$$
\forall z \in \mathcal{Z}_{1}, \quad i\{h, b\}(z)=2 \Re\left\langle-i \partial_{\bar{z}} h(z), \nabla_{\bar{z}} b(z)\right\rangle_{\mathcal{Z}_{1}}=\langle v(z), \nabla b(z)\rangle_{\mathcal{Z}_{1}, \mathbb{R}}
$$

holds for any $b \in \mathcal{S}_{\text {cyl }}\left(\mathcal{Z}_{1} ; \mathbb{R}\right)$ with $v(z)=-i \partial_{\bar{z}} h(z)$.

Proof. It suffices to compute

$$
\begin{aligned}
& i\{h, b\}=i\left[\partial_{z} h . \partial_{\bar{z}} b-\partial_{z} b . \partial_{\bar{z}} h\right] \\
& =i\left[\overline{\left\langle\partial_{\bar{z}} b, \partial_{\bar{z}} h\right\rangle_{\mathcal{Z}_{0}}}-\overline{\left\langle\partial_{\bar{z}} h, \partial_{\bar{z}} b\right\rangle_{\mathcal{Z}_{0}}}\right] \\
& =-2 \operatorname{Im}\left\langle\partial_{\bar{z}} h, \partial_{\bar{z}} b\right\rangle=2 \Re\left\langle-i \partial_{\bar{z}} h, \partial_{\bar{z}} b\right\rangle=2 \Re\left\langle-i \partial_{\bar{z}} h, \nabla_{\bar{z}} b\right\rangle_{\mathcal{Z}_{1}} \\
& =\left\langle-i \partial_{\bar{z}} h, \nabla b\right\rangle_{\mathcal{Z}_{1}, \mathbb{R}} \text {. }
\end{aligned}
$$


Proposition C.8. Let $\mathcal{Z}_{1} \subset \mathcal{Z}_{0} \subset \mathcal{Z}_{-1}$ be a Hilbert triple of separable complex Hilbert spaces. Consider a time dependent real sesquilinear form $z \rightarrow h(z, t)$ on $\mathcal{Z}_{1}$ which is Fréchet- $\mathcal{C}^{1}$ and such that $\mathcal{Z}_{1} \times \mathbb{R} \ni(z, t) \rightarrow\left(\partial_{\bar{z}} h(z, t), \partial_{z} h(z, t)\right) \in$ $\mathcal{Z}_{1} \times \mathcal{Z}_{-1}^{*}$ is strongly continuous. Assume also that the time-dependent Hamilton equation

$$
i \partial_{t} z_{t}=\partial_{\bar{z}} h\left(z_{t}, \bar{z}_{t}, t\right) \quad, \quad z_{t=s}=z
$$

admits a unique continuous solution $z_{t}=\Phi(t, s) z$ for all $t, s \in \mathbb{R}$ and all $z \in$ $\mathcal{Z}_{1}$, with $\Phi(t, s): \mathcal{Z}_{1} \rightarrow \mathcal{Z}_{1}$ Borel. Consider a time dependent measure $\mu(t) \in$ $\operatorname{Prob}_{2}\left(\mathcal{Z}_{1}\right)$ which satisfies

- $t \rightarrow \mu_{t} \in \operatorname{Prob}_{2}\left(\mathcal{Z}_{1}\right)$ is $W_{2}$-continuous,

- For all $T>0,\left|\partial_{\bar{z}} h(t)\right|_{L^{2}\left(\mathcal{Z}, \mu_{t}\right)} \in L^{1}([-T, T])$,

- The time-dependent probability measure $\mu_{t}$ is a weak solution to

$$
\partial_{t} \mu+i\{h(t), \mu\}=0,
$$

namely for all $\varphi \in \mathcal{C}_{0 \text {,cyl }}^{\infty}\left(\mathcal{Z}_{1} \times \mathbb{R} ; \mathbb{R}\right)$,

$$
\int_{\mathbb{R}} \int_{\mathcal{Z}_{1}}\left(\partial_{t} \varphi(z, t)+i\{h, \varphi\}(z, t)\right) d \mu_{t}(z) d t=0 .
$$

Then the measure $\mu_{t}$ satisfies

$$
\forall t, s \in \mathbb{R}, \quad \mu_{t}=\Phi(t, s)_{*} \mu_{s}
$$

and it is unique when $\mu_{0}$ is fixed.

Proof. We apply Proposition C.4 while $E=\mathcal{Z}_{1}$ is endowed with its Euclidean structure $\left\langle z_{1}, z_{2}\right\rangle \mathcal{Z}_{1}, \mathbb{R}=\Re\left\langle z_{1}, z_{2}\right\rangle \mathcal{Z}_{1}$. Lemma C.7 says that the weak Liouville equation is

$$
\forall \varphi \in \mathcal{C}_{0, \text { cyl }}^{\infty}\left(\mathcal{Z}_{1} \times \mathbb{R}\right), \quad \int_{\mathbb{R}} \int_{\mathcal{Z}_{1}}\left(\partial_{t} \varphi(z, t)+\langle v, \nabla \varphi\rangle(t, z)\right) d \mu_{t}(z) d t=0,
$$

with $v(z, t)=-i \partial_{\bar{z}} h(z, t)$. The measure $\mu_{t}$ is a weak solution to

$$
\partial_{t} \mu+\nabla^{T}(v \mu)=0
$$

where $\nabla$ and $\nabla^{T}$ are defined according to the real structure on $\mathcal{Z}_{1}$. Our hypotheses on $\mu$ and $h$ cover all the assumptions of Proposition C.4.

\section{Weak $L^{p}$ conditions for the potential $V$}

Let $L_{\mathbb{R}^{d}}$ be the Lebesgue measure on $\mathbb{R}^{d}$. For $0<p<\infty$, a Lebesgue measurable function $f: \mathbb{R}^{d} \rightarrow \mathbb{C}$ is said to belong to weak- $L^{p}\left(\mathbb{R}^{d}\right)$, or shortly to $L^{p, \infty}\left(\mathbb{R}^{d}\right)$, if there exists a constant $c>0$ such that for all $t>0$

$$
L_{\mathbb{R}^{d}}\{x:|f(x)|>t\} \leq c^{p} / t^{p} .
$$


Two functions in $L^{p, \infty}\left(\mathbb{R}^{d}\right)$ are equal if they are equal $L_{\mathbb{R}^{d}}$-almost everywhere. The quantity

$$
\begin{aligned}
\|f\|_{p, \infty} & =\inf \left\{c: L_{\mathbb{R}^{d}}\{x:|f(x)|>t\} \leq c^{p} / t^{p}, \forall t>0\right\} \\
& =\sup _{t>0}\left\{t L_{\mathbb{R}^{d}}\{x:|f(x)|>t\}^{1 / p}\right\}
\end{aligned}
$$

defines a complete quasi-norm on $L^{p, \infty}\left(\mathbb{R}^{d}\right)$ with $\|f\|_{p, \infty} \leq\|f\|_{p}$.

By combining the Hunt and Marcinkiewicz interpolation theorems according to $[11,34,56])$, the Young and Hölder inequalities can be extended to weak $L^{p}$ spaces:

Proposition D.1 (generalized Young inequality). Let $1<p, q, r<\infty$ such that $\frac{1}{p}+\frac{1}{q}=1+\frac{1}{r}$. There exists a constant $c_{p, q}>0$ such that for all $f \in L^{p}\left(\mathbb{R}^{d}\right)$ and $g \in L^{q, \infty}\left(\mathbb{R}^{d}\right)$

$$
\|f * g\|_{r} \leq c_{p, q}\|f\|_{p}\|g\|_{q, \infty} .
$$

Proposition D.2 (generalized Hölder inequality). Let $1<p, q, r<\infty$ satisfy $\frac{1}{p}+\frac{1}{q}=\frac{1}{r}$. There exists a constant $c_{p, q}$ such that for all $f \in L^{p, \infty}\left(\mathbb{R}^{d}\right)$ and $g \in L^{q}\left(\mathbb{R}^{d}\right)$

$$
\|f . g\|_{r} \leq c_{p, q}\|f\|_{p, \infty}\|g\|_{q} .
$$

Proposition D.3 (Hardy inequality). Suppose that $d \geq 3$ and $V \in L^{d, \infty}\left(\mathbb{R}^{d}\right)$. There exists a constant $c>0$ such that for all $u \in H^{1}\left(\mathbb{R}^{d}\right)$

$$
\|V u\|_{2} \leq c\|u\|_{H^{1}\left(\mathbb{R}^{d}\right)} .
$$

Proof. For $u \in L^{2}\left(\mathbb{R}^{d}\right)$, we can write $(1-\Delta)^{-1 / 2} u(x)=G * u(x)$ with $G$ the inverse Fourier transform of $\left(1+|x|^{2}\right)^{-1 / 2}$. It is not difficult to prove that $G \in$ $L^{\frac{d}{d-1}}, \infty$ (see [56, Exercice 50]). Hence, we conclude that

$$
\begin{aligned}
\left\|V(1-\Delta)^{-1 / 2} u\right\|_{2}=\|V G * u\|_{2} & \stackrel{\text { Hölder }}{\leq} C_{1}\|V\|_{d, \infty}\|G * u\|_{\frac{2 d}{d-2}} \\
& \stackrel{\text { Young }}{\leq} C_{2}\|V\|_{d, \infty}\|G\|_{\frac{d}{d-1}, \infty}\|u\|_{2} .
\end{aligned}
$$

The above proposition provides a class of potentials which are bounded multiplication operators from $H^{1}\left(\mathbb{R}^{d}\right)$ into $L^{2}(\mathbb{R})$ when $d \geq 3$. For lower dimension, the Sobolev embeddings give at once:

- if $d=1, V \in L^{2}(\mathbb{R})+L^{\infty}(\mathbb{R})$ then $V \in \mathcal{L}\left(H^{1}(\mathbb{R}), L^{2}(\mathbb{R})\right)$;

- if $d=2, V \in L^{p}\left(\mathbb{R}^{2}\right)+L^{\infty}\left(\mathbb{R}^{2}\right)$ for $p>2$, then $V \in \mathcal{L}\left(H^{1}\left(\mathbb{R}^{2}\right), L^{2}\left(\mathbb{R}^{2}\right)\right)$.

We denote by $L^{p}\left(\mathbb{R}^{d}\right)+L_{0}^{\infty}\left(\mathbb{R}^{d}\right)$ the space of Lebesgue measurable functions $f$ such that there exists $\left(f_{n}\right)_{n \in \mathbb{N}} \in L^{p}\left(\mathbb{R}^{d}\right)^{\mathbb{N}}$ satisfying $\lim _{n \rightarrow \infty}\left\|f-f_{n}\right\|_{\infty}=0$. 
Lemma D.4. For $0<p<q$,

$$
L^{q, \infty}\left(\mathbb{R}^{d}\right) \subset L^{p}\left(\mathbb{R}^{d}\right)+L_{0}^{\infty}\left(\mathbb{R}^{d}\right) .
$$

Proof. For $\epsilon>0$, decompose each $f \in L^{q, \infty}\left(\mathbb{R}^{d}\right)$ into a sum $f=f_{\epsilon}+f^{\epsilon}$ such that $f_{\epsilon}=f 1_{|f|>\epsilon}$ and $f^{\epsilon}=f 1_{|f| \leq \epsilon}$. Observe that for any $\epsilon>0$

$$
\begin{aligned}
\left\|f_{\epsilon}\right\|_{p}^{p} & =p \int_{0}^{\infty} t^{p-1} L_{\mathbb{R}^{d}}\left\{x:\left|f_{\epsilon}(x)\right|>t\right\} d t \\
& =p \int_{\epsilon}^{\infty} t^{p-1} L_{\mathbb{R}^{d}}\{x:|f(x)|>t\} d t+\epsilon^{p} L_{\mathbb{R}^{d}}\{x:|f(x)|>\epsilon\} \\
& \leq c \int_{\epsilon}^{\infty} \frac{t^{p-1}}{t^{q}} d t+\epsilon^{p} L_{\mathbb{R}^{d}}\{x:|f(x)|>\epsilon\}<\infty .
\end{aligned}
$$

Moreover, when $\epsilon \rightarrow 0$

$$
\left\|f^{\epsilon}\right\|_{\infty}=\left\|f 1_{|f| \leq \epsilon}\right\|_{\infty} \leq \epsilon \rightarrow 0 .
$$

Therefore, each $f \in L^{q, \infty}\left(\mathbb{R}^{d}\right)$ belongs to the space $L^{p}\left(\mathbb{R}^{d}\right)+L_{0}^{\infty}\left(\mathbb{R}^{d}\right)$.

Proposition D.5. For any $V \in L^{2}\left(\mathbb{R}^{d}\right)+L_{0}^{\infty}\left(\mathbb{R}^{d}\right)$ such that $V(1-\Delta)^{-1 / 2} \in$ $\mathcal{L}\left(L^{2}\left(\mathbb{R}^{d}\right)\right)$ the operator $(1-\Delta)^{-1 / 2} V(1-\Delta)^{-1 / 2}$ is compact.

Proof. Let $g(\xi)=\left(1+|\xi|^{2}\right)^{-1 / 2}$ and $g_{m}(\xi)=1_{[0, m]}(|\xi|) g(\xi)$. The following norm convergence holds

$$
\lim _{m \rightarrow \infty} g_{m}(D) V g_{m}(D)=g(D) V g(D)
$$

using the fact that $\lim _{m \rightarrow \infty}\left\|g_{m}(D)-g(D)\right\|_{\mathcal{L}\left(L^{2}\left(\mathbb{R}^{d}\right)\right)}=0$ and $\|V g(D)\|_{\mathcal{L}\left(L^{2}\left(\mathbb{R}^{d}\right)\right)}<\infty$.

By Lemma D.4, there exist $V_{n} \in L^{2}\left(\mathbb{R}^{d}\right)$ such that $\lim _{n \rightarrow \infty}\left\|V-V_{n}\right\|_{\infty}=0$. We observe now that the Hilbert-Schmidt norm of $g_{m}(D) V_{n}(x) g_{m}(D)$ is

$$
\left\|g_{m}(D) V_{n} g_{m}(D)\right\|_{\mathcal{L}^{2}\left(L^{2}\left(\mathbb{R}^{d}\right)\right)} \leq\left\|V_{n}\right\|_{2}\left\|g_{m}\right\|_{2}^{2}<\infty .
$$

Therefore, by norm convergence, the operator $g(D) V g(D)$ is compact.

Corollary D.6. The potential $V$ satisfies the assumptions (A2)-(A3) in the following cases:

- if $d=1$ and $V \in L^{2}(\mathbb{R})+L_{0}^{\infty}(\mathbb{R})$,

- if $d=2$ and $V \in L^{p}\left(\mathbb{R}^{2}\right)+L_{0}^{\infty}\left(\mathbb{R}^{2}\right)$ with $p>2$,

- if $d \geq 3$ and $V \in L^{d, \infty}\left(\mathbb{R}^{d}\right)$.

Proof. Combine Proposition D.3, Lemma D.4 and Proposition D.5 with, in dimension $d=2$, the observation

$$
L^{p}\left(\mathbb{R}^{2}\right)+L_{0}^{\infty}\left(\mathbb{R}^{2}\right) \subset L^{2}\left(\mathbb{R}^{2}\right)+L_{0}^{\infty}\left(\mathbb{R}^{2}\right) \quad \text { for } p>2 .
$$

In particular, in dimension $d=3$ the Coulomb potential $V(x)= \pm \frac{1}{|x|}$ satisfies the assumptions (A1), (A2) and (A3) because $\frac{1}{|x|} \in L^{3, \infty}\left(\mathbb{R}^{3}\right)$. 


\section{References}

[1] A. Aftalion, "Vortices in Bose-Einstein Condensates", Progress in Nonlinear Differential Equations and their Applications, Vol. 67, Birkhaüser, 2006.

[2] L. Ambrosio, A. Figalli, G. Friesecke, J. Giannoulis and T. Paul, Semiclassical limit of quantum dynamics with rough potentials and well-posedness of transport equations with measure initial data, Comm. Pure Appl. Math. 64 (2011), 1199-1242.

[3] L. Ambrosio, N. Gigli and G. Savaré, "Gradient Flows in Metric Spaces and in the Space of Probability Measures", Lectures in Mathematics ETH Zürich, Birkhäuser Verlag, 2005.

[4] Z. AMMARI and F. NIER, Mean field limit for bosons and infinite-dimensional phase-space analysis, Ann. Henri Poincaré 9 (2008), 1503-1574.

[5] Z. AMMARI and F. NIER, Mean field limit for bosons and propagation of Wigner measures, J. Math. Phys. 50 (2009).

[6] Z. AMMARI and F. NIER, Mean field propagation of Wigner measures and BBGKY hierarchies for general bosonic states J. Math. Pures Appl. 95 (2011), 585-626.

[7] I. Anapolitanos, Rate of convergence towards the Hartree von Neumann limit in the mean-field regime, Lett. Math. Phys. 98 (2011), 1-31.

[8] C. BARdos, F. GOLSE and N. MAUSER, Weak coupling limit of the n-particle Schrödinger equation, Methods Appl. Anal. 7 (2000), 275-293.

[9] C. Bardos, L. Erdös, F. Golse, N. Mauser and H-T. Yau Derivation of the Schrödinger-Poisson equation from the quantum N-body problem, C.R. Math. Acad. Sci. Paris 334 (2002), 515-520.

[10] F. A. BerEZIN, "The Method of Second Quantization", Second edition, "Nauka", Moscow, 1986.

[11] J. BerGH and J. LÖFSTRÖM, "Interpolation Spaces. An Introduction", Grundlehren der Mathematischen Wissenschaften, No. 223. Springer-Verlag, 1976.

[12] J. M. BONY and N. LERNER, Quantification asymptotique et microlocalisation d'ordre supérieur I, Ann. Scient. Éc. Norm. Sup. (4) 22 (1989), 377-433.

[13] N. BuRQ, "Mesures semi-classiques et mesures de défaut", Séminaire Bourbaki, Vol. 39 (1996-1997), Exposé No. 826, 29 p.

[14] N. BURQ, Semiclassical estimates for the resolvent in non trapping geometries, Int. Math. Res. Notices 5 (2002), 221-241.

[15] F. CAStella, T. JeCKO and A. KnAUF, Semiclassical resolvent estimates for Schrödinger operators with Coulomb singularities, Ann. Henri Poincaré 9 (2008), 775-815.

[16] T. CaZenave, "Semilinear Schrödinger Equations", Courant Lecture Notes in Mathematics, Vol. 10, AMS, 2003.

[17] T. CHEN and N. PAVLOVIĆ, The quintic NLS as the mean field limit of a boson gas with three-body interactions, J. Funct. Anal. 260 (2011), 959-997.

[18] J. DeREZINSKi, Asymptotic completeness in quantum field theory. A class of Galilei covariant models, Rev. Math. Phys. 10 (1998), 191-233.

[19] L. ERDÖs and H.T. YAU, Derivation of the nonlinear Schrödinger equation from a many body Coulomb system, Adv. Theor. Math. Phys. 5 (2001), 1169-2005.

[20] L. ERdös, B. SCHLEIN and H.T. YAU, Derivation of the cubic non-linear Schrödinger equation from quantum dynamics of many-body systems, Invent. Math. 167 (2007), 515614.

[21] L. ERdös, B. SCHLEIN and H.T. YAU, Derivation of the Gross-Pitaevskii equation for the dynamics of Bose-Einstein condensate, Ann. of Math. (2) 172 (2010), 291-370.

[22] C. Fermanian-KAMmerer and P. GÉRARD, Mesures semi-classiques et croisement de modes, Bull. Soc. Math. France 130 (2002), 123-168.

[23] G. B. Folland, "Harmonic Analysis in Phase Space", Annals of Mathematics Studies, Vol. 122, Princeton University Press, 1989. 
[24] G. B. Folland, "Quantum Field Theory: A Tourist Guide for Mathematicians", Mathematical Surveys and Monographs, AMS, Vol. 149, 2008.

[25] J. FRÖHLICH, S. GRAFFI and S. SCHWARZ, Mean-field- and classical limit of many-body Schrödinger dynamics for bosons, Comm. Math. Phys. 271 (2007), 681-697.

[26] J. Fröhlich, A. KnOwles and A. Pizzo, Atomism and quantization, J. Phys. A 40 (2007), 3033-3045.

[27] J. Fröhlich, A. KNowles and S. Schwarz On the Mean-field limit of bosons with Coulomb two-body interaction, Comm. Math. Phys. 288 (2009), 1023-1059.

[28] P. GÉrARD, Microlocal defect measures, Comm. Partial Differential Equations 16 (1991), 1761-1794.

[29] P. GÉRARD, Mesures semi-classiques et ondes de Bloch, In: "Séminaire sur les Équations aux Dérivées Partielles”, 1990-1991,Exp. No.XVI, 19 pp., École Polytech., Palaiseau, 1991.

[30] P. GÉrard, P. A. MARKowich, N. J. MaUser and F. Poupaud, Homogenization limits and Wigner transforms, Comm. Pure Appl. Math. 50 (1997), 323-379.

[31] J. GiNIBRE, "Equations d'évolution semilinéaires: L'équation de Schrödinger nonlinéaire", Lecture Notes in Université de Paris-Sud, 1994.

[32] J. Ginibre and G. Velo, The classical field limit of scattering theory for nonrelativistic many-boson systems. I, Comm. Math. Phys. 66 (1979), 37-76.

[33] J. GinibRE and G. VELO, The classical field limit of scattering theory for nonrelativistic many-boson systems. II, Comm. Math. Phys. 68 (1979), 45-68.

[34] L. GrafaKos, "Classical Fourier Analysis", Second edition. Graduate Texts in Mathematics, Vol. 249, Springer, 2008.

[35] S. Graffi, A. Martinez and M. Pulvirenti, Mean-field approximation of quantum systems and classical limit, Math. Models Methods Appl. Sci. 13 (2003), 59-73.

[36] M. HAURAY and P. E. JABIN, N-particles approximation of the Vlasov equations with singular potential, Arch. Ration. Mech. Anal. 183 (2007).

[37] B. HelfFer, A. MARTINEZ and D. RoBERT, Ergodicité et limite semi-classique, Comm. Math. Phys. 109 (1987), 313-326.

[38] B. HelfFER and H. Siedentop, A generalization of the Kustaanheimo-Stiefel transform for two-centre systems, Bull. London Math. Soc. 28 (1996), 33-42.

[39] K. HEPP, The classical limit for quantum mechanical correlation functions, Comm. Math. Phys. 35 (1974), 265-277.

[40] L. HöRmANDER, "The Analysis of Linear Partial Differential Operators", Springer Verlag, 1985.

[41] S. KeRAANI, Wigner measures dynamics in a Coulomb potential, J. Math. Phys. 46 (2005), $21 \mathrm{p}$.

[42] S. Klainerman and M. Machedon, On the uniqueness of solutions to the GrossPitaevskii hierarchy, Comm. Math. Phys. 279 (2008).

[43] A. KNAUF, The n-centre problem of celestial mechanics for large energies, J. Europ. Math. Soc. (JEMS) 4 (2002), 1-114.

[44] A. KNOWLES and P. PICKL Mean-field dynamics: singular potentials and rate of convergence, Comm. Math. Phys. 298 (2010), 101-138.

[45] P. KRÉE and R. RACZKA, Kernels and symbols of operators in quantum field theory, Ann. Inst. H. Poincaré Sect. A (N.S.) 28 (1978), 41-73.

[46] P. KustaAnHEIMO, Perturbation theory of Kepler motion based on spinor regularization, J. Reine Angew. Math. 218 (1965), 204-219.

[47] B. LASCAR, Une condition nécessaire et suffisante d'éllipticité pour une classe d'opérateurs différentiels en dimension infinie, Comm. Partial Differential Equations 2 (1977), 3167.

[48] E.H. Lieb, R. Seiringer, J.P. SoloveJ and J. Yngvason, "The Mathematics of the Bose Gas and its Condensation", Birkhäuser, 2005.

[49] P. L. Lions and T. PAUl, Sur les mesures de Wigner, Rev. Mat. Iberoamericana 9 (1993), 553-618. 
[50] A. Martinez, "An Introduction to Semiclassical Analysis and Microlocal Analysis", Universitext, Springer-Verlag, 2002.

[51] L. MiLlER, Short waves through thin interfaces and 2-microlocal measures, In: Journées "Équations aux Dérivées Partielles" (Saint-Jean-de-Monts, 1997), Exp. No. XII, 12 pp., École Polytech., Palaiseau, 1997.

[52] C. Mouhot and C. Villani, Landau Damping, Acta Math. 207 (2011), 29-201.

[53] F. NATAF and F. NIER, Convergence of domain decomposition methods via semi-classical calculus, Comm. Partial Differential Equations 23 (1998), 1007-1059.

[54] F. NIER A semi-classical picture of quantum scattering, Ann. Sci. Éc. Norm. Sup. (4) 29 (1996), 149-183.

[55] K. R. Parthas arathy, "Probability Measures on Metric Spaces", Probability and Mathematical Statistics, No. 3, Academic Press, 1967.

[56] M. ReED and B. Simon, "Methods of Modern Mathematical Physics", Vol. II, Academic Press, 1976.

[57] D. ROBERT, “Autour de l'approximation semi-classique", Progress in Mathematics, Vol.68, Birkhäuser, 1987.

[58] I. RODNIANSKI and B. SCHLEIN, Quantum fluctuations and rate of convergence towards mean field dynamics, Comm. Math. Phys. 291 (2009), 31-61.

[59] A. V. SKOROHOD, "Integration in Hilbert Space", Ergebnisse der Mathematik und ihrer Grenzgebiete, Band 79. Springer-Verlag, 1974.

[60] H. SpOHN, Kinetic equations from Hamiltonian dynamics, Rev. Mod. Phys. 52 (1980), 569-615.

[61] L. TARTAR, H-measures, a new approach for studying homogenization, In: "Oscillations and Concentration Effects in Partial Differential Equations", Proc. Roy. Soc. Edinburgh 115-A (1990), 193-230.

[62] C. Villani, "Topics in Optimal Transportation", Graduate Studies in Mathematics, Vol. 58, AMS, 2003.

IRMAR

Université de Rennes I

UMR-CNRS 6625

campus de Beaulieu

35042 Rennes Cedex, France

zied.ammari@univ-rennes1.fr

LAGA, UMR-CNRS 7539

Université de Paris 13

99, avenue Jean-Baptiste Clément

93430 Villetaneuse, France

nier@math.univ-paris13.fr 